\title{
The Geometry of the Compactification of the Hurwitz Scheme
}

\author{
By
}

\author{
Shinichi Mochizuki* \\ Table of Contents
}

Table of Contents

Introduction

$\S 1$. Different Types of Hurwitz Schemes

\$2. Irreducibility

§3. Log Admissible Coverings

$\S 3$ A. Basic Definitions

$\S 3$ B. First Properties

§3C. Global Moduli

$\S 3 \mathrm{D}$. Admissible Hurwitz Coverings

$\S 4$. Construction of the Boundary Components

Appendix to $\S 4$

Pictorial Appendix

$\S 5$. Cohomology Calculations

$\S 6$. The Main Fibration

$\S 6 \mathrm{~A}$. The Excess Divisors in the Main Fibration

$\S 6 \mathrm{~B}$. Intersection Theory Calculations

$\S 6 \mathrm{C}$. Ramification Indices

§7. The Coefficient Matrix

§8. Arithmetic Applications

Bibliography

\section{Imtroduction}

The purpose of this paper is to study the geometry of the Harris-Mumford compactification of the Hurwitz scheme. The Hurwitz scheme parametrizes certain ramified coverings $f: C \rightarrow \mathbb{P}^{1}$ of the projective line by smooth curves. Thus, from the very outset, one sees that there are essentially two ways to approach the

Received June 11, 1993.

1991 Mathematics Subject Classification: $14 \mathrm{H} 10$

*Research Institute for Mathematical Sciences, Kyoto University, Kyoto 606, Japan 
Hurwitz scheme:

(1) We start with $\mathbb{P}^{1}$ and regard the objects of interest as coverings of $\mathbb{P}^{1}$;

(2) We start with $C$ and regard the objects of interest as morphisms from $C$ to $\mathbb{P}^{1}$.

One finds that one can obtain the most information about the Hurwitz scheme and its compactification by exploiting interchangeably these two points of view.

Our first main result is the following

Theorem. Let $b, d$, and $g$ be integers such that $b=2 d+2 g-2, g \geq 5$ and $d>2 g+4$. Let $\mathscr{H}$ be the Hurwitz scheme over $\mathbb{Z}\left[\frac{1}{b !}\right]$ parametrizing coverings of the projective line of degree $d$ with $b$ points of ramification. Then $\operatorname{Pic}(\mathscr{H})$ is finite.

Remark. The number $g$ in the statement of the Theorem is the genus of the "curve $C$ upstairs" of the coverings in question. Note, however, that the Hurwitz scheme $\mathscr{H}^{\prime}$, and hence also the genus $g$, are completely determined by $b$ and $d$.

This Theorem is stated in $\$ 6.7$, of the text. Note that although in the statement of the Theorem here in the introduction, we spoke of "the" Hurwitz "scheme," there are in fact several different Hurwitz schemes used in the literature, some of which are, in fact, not schemes, but stacks. For details about the particular type of Hurwitz scheme for which the main theorem is proved, we refer the reader to the exact statement in $\S 6.7$, as well as to $\S 1$ which explains the notation. Finally, we should address the issue of what happens if $d \leq 2 g+4$. Although our proof uses the somewhat leisurely lower bound of $2 g+4$, it may be possible to prove the same result for smaller $d$ using essentially the same techniques, but being just a bit more careful. Since at the time this paper was written, the author was not particularly interested in this issue, he has allowed himself the luxury of taking $d$ to be greater than $2 g+4$.

The main idea of the proof is that by combinatorially analyzing the boundary of the compactification of the Hurwitz scheme, one realizes that there are essentially three kinds of divisors in the boundary, which we call excess divisors (§4.7), which are "more important" than the other divisors in the boundary in the sense that the other divisors map to sets of codimension $\geq 2$ under various natural morphisms. On the other hand, we can also consider the moduli stack $\mathscr{G}(\$ 6.1)$ of pairs consisting of a smooth curve of genus $g$, together with a linear system of degree $d$ and dimension 1 . The subset of $\&$ consisting of those pairs that arise 
from Hurwitz coverings is open in $\mathscr{G}$, and its complement consists of three divisors, which correspond precisely to the excess divisors. Using results of Harer on the Picard group of $\mathscr{M}_{g}$, we show that these three divisors on $\mathscr{G}$ form a basis of $\operatorname{Pic}(\mathscr{G}) \otimes_{\mathbf{Z}} \mathbb{Q}$, and in fact, we even compute explicitly $(\S 7)$ the matrix relating these three divisors on $\mathscr{G}$ to a certain standard basis of $\operatorname{Pic}(\mathscr{G}) \otimes_{\mathbf{z}} \mathbf{Q}$. The above Theorem then follows formally.

Crucial to our study of the Hurwitz scheme is its compactification by means of admissible coverings, a notion introduced in [19]. In [19], the existence of a coarse moduli scheme of such coverings is stated. However, we could not follow a certain key step in the construction of this coarse moduli scheme, and so we decided that it would be best to give a treatment of such coverings independent of [19]. In fact, we study a more general sort of admissible covering than [19], between stable curves of arbitrary genus, and we prove a rather general theorem (\$3.22) concerning the existence of a canonical logarithmic (in the sense of [21]) algebraic stack $(\mathscr{\odot}, M$,$) parametrizing such coverings:$

Theorem. Fix nonnegative integers $g, r, q, s, d$ such that $2 g-2+r=d(2 q-$ $2+s) \geq 1$. Let $\mathscr{N}$ be the stack over $\mathbb{Z}$ defined as follows: For a scheme $S$, the objects of $\mathscr{\alpha}(S)$ are admissible coverings $\pi: C \rightarrow D$ of degree $d$ from a symmetrically r-pointed stable curve $\left(f: C \rightarrow S ; \mu_{f} \subseteq C\right)$ of genus $g$ to a symmetrically s-pointed stable curve $\left(h: D \rightarrow S ; \mu_{h} \subseteq D\right)$ of genus $q$; and the morphisms of $\mathscr{N}(S)$ are pairs of S-isomorphisms $\alpha: C \rightarrow C$ and $\beta: D \rightarrow D$ that stabilize the divisors of marked points such that $\pi \circ \alpha=\beta \circ \pi$. Then $\mathscr{A}$ is a separated algebraic stack of finite type over $\mathbb{Z}$. Moreover, $\mathscr{A}$ is equipped with a canonical log structure $M_{,} \rightarrow \mathbb{C}_{,}$, together with a logarithmic morphism $(\Omega, M,) \rightarrow \overline{\mathscr{L}}_{4, s}^{\log }$ (obtained by mapping $\left.(C ; D ; \pi) \mapsto D\right)$ which is log étale (always) and proper over $\mathbb{Z}\left[\frac{1}{d !}\right]$.

Now we summarize what we do section by section. In $\S 1$, we define various Hurwitz schemes as well as certain auxiliary objects to be used later. All of these objects have both "combinatorial" (corresponding to the first point of view) and "algebro-geometric" (corresponding to the second point of view) definitions. In $\S 2$, we use the combinatorial point of view to prove the irreducibility of many of the objects of $\S 1$. In $\S 3$, we discuiss admissible coverings from the point of view of log schemes, and prove the Theorem just stated above. In $\$ 4$, the culmination of our exploitation of the combinatorial point of view, we explicitly enumerate and construct the divisors at infinity of the compactification, and begin the determination of the divisor class group of the Hurwitz scheme. 
From then on, we switch gears to the second, or more algebro-geometric, point of view. In $\$ 5$, we prepare for this by reviewing certain relevant cohomological results. In $\S 6$, we carry out a detailed study of the "excess divisors" at infinity and thereby complete the proof of the finiteness of the divisor class group of the Hurwitz scheme, modulo a technical result from the next Section. Finally, in $\$ 7$, to make our understanding of the excess divisors more explicit, we carry out certain calculations relating the excess divisors to other, better known line bundles, which also serve to complete the proof of the technical result needed earlier.

$\S 8$ is purely conjectural and proposes possible applications of the results discussed previously to prove arithmetic results. In particular, the explicit calculations of $\$ 7$ suggest a possible application to an effective form of the Mordell conjecture.

The reader who is interested in the circle of ideas dealt with in this paper may also consult the related work of Arbarello, Harris, and Diaz on Severi varieties; see, for instance, [18]. Although the results of [18] are not literally the same as ours, they are certainly closely philosophically related. Also, D. Edidin has been preparing a paper that gives similar results, although at the present time, I have not yet seen this paper. Finally, although we treat here the case when the dimension of the Hurwitz scheme is rather large, when the dimension is very small (i.e., 1 or 2), one has the results of [6] and [7].

An earlier version of this paper was submitted as my doctoral dissertation at Princeton University in the spring of 1992. I would like to thank my advisor, Prof. G. Faltings, both for suggesting the topic and for his advice and support during my years as a graduate student. Also, although none of our conversations contributed directly to the material in this paper, my general understanding of algebraic geometry profited greatly from my numerous conversations with Prof. N. Katz; I would, therefore, like to express here my thanks to him, as well. Next, I would like to thank Prof. K. Kato for explaining the notion of a log structure to me during the summer of 1991 . Finally, I would like to express my deep gratitude to Profs. K. Saito and Y. Ihara for encouraging me to publish this paper, despite substantial opposition in certain parts of the algebraic geometry community to the use of stacks.

\section{$\S 1$. Differemt Types of Hurwitz Schemes}

$\S 1.1$. The original purpose of the Hurwitz scheme is to parametrize coverings of the projective line that have at most simple branch points. However, since there are several different versions of the Hurwitz schemes running around in the 
literature, we take the opportunity here to standardize and make explicit which version we are using at any particular time. Also, it is necessary to construct Hurwitz-type schemes which parametrize coverings with worse ramification than "simple branch points". These generalized Hurwitz schemes will aid in our elucidation of the geometry at infinity of the ordinary Hurwitz scheme. We shall omit some details here since we are essentially reviewing well-known material. For more details, see [13].

$\S 1.2$. Remark on characteristic $p$ : In general, in this part of the paper, we will work over the ring $\mathbb{Z}\left[\frac{1}{N}\right]$, where $N$ is divisible by all numbers characteristic to the problem. Thus there will be no essential difference between what we do and what one would do if one were to work over characteristic zero. Indeed, we shall often prove some results by reducing to the case of characteristic zero.

Remark on Stacks: We will need to employ the notion of an "algebraic stack" (which we will henceforth call simply a "stack" for short) in the sense of Deligne-Mumford [8]; the reader who is not satisfied with the treatment given in [8] may also refer to Chapter $1, \S 4$, of [11] for basic facts about stacks. In fact, (see [11], Chapter 1, §4.10) étale locally, every stack can be formed by taking quotients (in the sense of stacks) of schemes by finite group actions. We explain what this means as follows: Let $S$ be a noetherian scheme; let $X$ be an $S$-scheme of finite type; let $G$ be a finite group acting on $X$ by means of $S$-automorphisms. Then we shall denote by $[X / G]$ the algebraic stack defined by "taking the quotient of $X$ by $G$ in the sense of stacks."

Concretely, relative to the "Working Definition of Algebraic Stacks" given in [11], Chapter $1, \S 4.9,[X / G]$ is defined as follows: In the notation of loc. cit., it suffices to specify schemes $R$ and $U$, together with morphisms $s, t: R \rightarrow U$, and $\mu: R \times_{U, t, s} R \rightarrow R$. For $U$, we take $X$; for $R$, we take $G \times X$ (i.e., a disjoint union of copies of $X$ indexed by the elements of $G$ ). For $t: R \rightarrow U$, we take the morphism $G \times X \rightarrow X$ that defines the group action. For $s: R \rightarrow U$, we take the projection $G \times X$ on the second factor. For $\mu: R \times_{U, t, S} R=(G \times X) \times_{X}(G \times X) \rightarrow$ $R=G \times X$, we take the morphism that sends $\left(g_{1}, x\right) \times\left(g_{2}, g_{1} x\right)$ to $\left(g_{2}, g_{1} x\right)$. One checks easily that all the necessary hypotheses are satisfied.

Now let us suppose that $X=\operatorname{Spec}(A)$ is affine. In [11], Theorem 4.10, a general method is described for passing from a noetherian algebraic stack to an associated coarse moduli space (which in general is just an algebraic space, not necessarily a scheme). If one applies this Theorem to the algebraic stack $[X / G]$ just constructed, one sees easily that one obtains the scheme $Y=\operatorname{Spec}\left(A^{G}\right)$ 
(where $A^{G} \subseteq A$ is the subring of functions invariant under the action of $G$ ) as the coarse moduli space associated to $[X / G]$.

$\S 1.3$. We start with the ordinary Hurwitz scheme. Let $b, d$, and $g$ be natural numbers subject to the relation $2(g-1)=-2 d+b$, with $b \geq 4$. Psychologically, $g$ is the genus of the curve upstairs, $b$ is the number of branch point of the covering, and $d$ is the degree of the covering. Suppose we wish to parametrize sets of $b$ distinct points of $\mathbb{P}^{1}$. Let $R=\mathbb{Z}\left[\frac{1}{b !}\right]$. (The reason for inverting these primes is so that all of our coverings of degree $b$ or $d$ will have tame ramification, as well as Galois closures which are generically separable.) Then there are (at least) four ways of doing this: we consider the category $\mathscr{E}$ of $R$-schemes and the stack (in fact, a covariant functor in the first three cases) on $\mathscr{E}$, that assigns to $S$ (an object of $\mathscr{C}$ ) one of the four following categories (or sets in the first three cases):

(1) " $U O_{b} "$ (= "unrigidified ordered"): ordered sets of sections $\sigma_{1}, \ldots \sigma_{b}$ : $S \rightarrow S \times \mathbb{P}^{1}$ (i.e., such that composing further with the first projection $S \times \mathbb{P}^{1} \rightarrow S$ is the identity) such that the images of $\sigma_{1}$ and $\sigma_{J}$ do not intersect when $i \neq j$;

(2) "US " (= "unrigified symmetrized"): divisors $D \subseteq S \times \mathbb{P}^{1}$ étale over $S$ of degree $b$;

(3) " $\mathrm{RO}_{b}$ " (= "rigidified ordered"): isomorphism classes of ordered sets of sections as in (1), where by "isomorphism" we mean that we regard $\left\{\sigma_{1}, \ldots, \sigma_{b}\right\}$ as isomorphic to $\left\{\sigma_{1}^{\prime}, \ldots, \sigma_{b}^{\prime}\right\}$ if there exists an $S$-automorphism of $S \times \mathbb{P}^{1}$ that carries $\left\{\sigma_{1}, \ldots, \sigma_{b}\right\}$ to $\left\{\sigma_{1}^{\prime}, \ldots, \sigma_{b}^{\prime}\right\}$;

(4) " $R S_{b}$ " (= "rigidified symmetrized"): the stack whose objects are étale divisors in $\mathbb{P}^{1}$-bundles (in the étale topology) over $S$, and whose morphisms are isomorphisms of $\mathbb{P}^{1}$-bundles that preserve the designated divisors. Put another way, this stack is the stack obtained by taking the quotient (in the sense of stacks) of the functor defined in (3) by the natural action of $\mathscr{S}^{b}$ (the symmetric group on $b$ letters) given by permuting the marked sections.

Although the use of the terminology "rigidified" here may at first appear counterintuitive in the sense that often one adds a "rigidifying structure," then forms the quotient by the action of some algebraic group, here we chose to use this terminology in the sense that "rigidification" consists in fixing the first three 
points at 0,1 , and $\infty$, whereas in the "unrigidified situation" all the points are floating around freely, hence not fixed or rigidified.

It is elementary that each of the above four functors is representable by a smooth, quasi-compact stack (in fact, a scheme for the first three functors) over $R$. Namely:

(1) $\mathscr{U} C_{\mathrm{b}}$ : Here we simply take $\left(\mathbf{P}^{\mathfrak{l}}\right)^{b}-\{$ diagonals $\}$.

(2) $\mathscr{U} \mathscr{S}_{\mathrm{b}}:$ We form the quotient by the action of $\mathscr{S}^{\mathrm{b}}$, the symmetric group on $b$ letters acting on $\mathscr{U} O_{\mathrm{b}}$ by permuting the $b$ factors of $\mathbb{P}^{1}$, to obtain the appropriate scheme, which may be naturally regarded as an open subset of $\mathbb{P}^{b}$, namely, the complement of the discriminant locus.

(3) $\mathscr{R} \mathscr{Q}_{\mathrm{b}}$ : For $1 \leq i, j \leq b-3$ with $i \neq j$, let $\Delta_{l j} \subseteq\left(\mathbb{P}^{1}\right)^{b-3}$ be the $(i, j)$-diagonal. Let $p_{l}:\left(\mathbf{P}^{1}\right)^{b-3} \rightarrow \mathbb{P}^{1}$ for $1 \leq i \leq b-3$ be the $i^{\text {th }}$ projection. Then

$$
\left(\mathbb{P}^{1}\right)^{b-3}-\left\{\left(\bigcup_{l \neq J} \Delta_{l J}\right) \cup\left(\bigcup_{l} p_{l}^{-1}(\{0,1, \infty\})\right)\right\}
$$

does the job.

(4) $\mathscr{R}_{\mathrm{b}}$ : Clearly $\mathscr{S}^{\mathrm{b}}$ acts on $\mathscr{R} \mathscr{C}_{\mathrm{b}}$ in such a way that the action is generically free. If we form the quotient by this action in the sense of stacks (as reviewed in $\S 1.2$ ), we obtain the desired algebraic stack, which is generically a scheme.

We will refer to any one of these four schemes (resp. functors, stacks) as " $b$ point schemes (resp. functors, stacks)", prefixing this term with the appropriate descriptives "rigidified", "ordered", etc. when necessary, and omitting the " $b$ " when speaking generally. These four $b$-point stacks fit into the following commutative diagram:

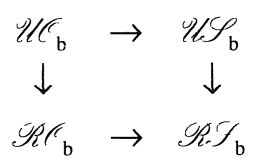

(Diagram 1.1A)

Note that the vertical arrows are $P G L(2)$-torsors, while the horizontal arrows are $\mathcal{S}^{\mathrm{b}}$-torsors. Thus, when we tensor with $\mathbf{C}$ and take the topological fundamental group, we get:

$$
\begin{array}{ccc}
\pi_{1}^{t o p}\left(\mathscr{H} C_{\mathrm{b}, \mathrm{C}}\right) & \rightarrow & \pi_{1}^{t o p}\left(\mathscr{H}_{\mathrm{b}, \mathrm{C}}\right) \\
\downarrow & & \downarrow \\
\pi_{1}^{t o p}\left(\mathscr{H}_{\mathrm{P}, \mathrm{C}}\right) & \rightarrow & \pi_{1}^{t o p}\left(\mathscr{M P S}_{\mathrm{b}, \mathrm{C}}\right)
\end{array}
$$


Here the horizontal arrows are injections and the vertical arrows are surjections; also, by "topological fundamental group," we mean in the sense of stacks, i.e., the fundamental group formed by considering étale coverings of the stack in question by analytic stacks. Finally, let us note that $\mathscr{U} \mathscr{S}_{\mathrm{b}}$ is used in [13], while $\mathscr{R} \mathscr{O}_{\mathrm{b}}$ is used in [19].

Let $T$ be any one of the four $b$-point stacks (resp. schemes). Then to $T$, we may associate a Hurwitz stack $H$ in such a way that $H$ is étale over $T$, hence representable by an algebraic stack. Indeed, consider the following stacks (which are schemes in the first three cases, so long as $d \geq 2$ ) over $\mathscr{E}$ :

(1) $\mathscr{H}^{\prime} \mathbb{C}_{\mathrm{b} . \mathrm{d}}$ : data of the following form: an arrow $\alpha: C \rightarrow \mathbb{P}^{1}$ in the category of $S$-schemes such that the induced arrow $C \rightarrow S$ is a smooth, geometrically connected, proper curve of genus $g$, and where $\alpha$ is flat of degree $d$ with simple ramification (i.e., the discriminant divisor is étale over the base - see [13], §5) exactly at given sections $\sigma_{1}, \ldots, \sigma_{b}: S \rightarrow \mathbb{P}^{1}$, where the $\sigma_{i}$ 's are mutually disjoint $S$-sections;

(2) $\mathscr{H}_{\mathcal{C}} \mathscr{S}_{\text {b.d }}$ : same sort of data as above except that instead of the $\sigma_{1}$ 's, we are given a divisor $D \subseteq S \times \mathbb{P}^{1}$ which is finite étale over $S$ of degree $d$, at which the simple ramification is to take place;

(3) $\mathscr{H} R C_{\mathrm{b}, \mathrm{d}}$ : isomorphism classes of the data in (1), where isomorphisms involve automorphisms over $S$ of $\mathbb{P}^{1}$ that carry one set of sections to the other, and over which there is an isomorphism of the respective curves $C$;

(4) $\mathscr{H}_{\mathscr{S}} \mathscr{S}_{\text {b,d }}$ : stack whose objects are collections of data as in (2), except that we replace $S \times \mathbb{P}^{1}$ by an arbitrary $\mathbb{P}^{1}$-bundle in the étale topology, and whose morphisms are isomorphisms of $\mathbb{P}^{1}$-bundles that preserve the designated divisors and over which lies an isomorphism of the respective curves $C$.

We see easily by Grothendieck's representability theorem (as applied in [13], §6) that all four of these Hurwitz stacks are relatively representable by étale morphisms over their respective $b$-point schemes. The key fact in the proof of loc. cit. (and proven there on p. 566) is the following result, whose proof we repeat here for the convenience of the reader (in a slightly more general form):

Lemma. Let $k$ be an algebraically closed field, $f: C \rightarrow \mathbb{P}_{k}^{1}$ a covering of degree $d \geq 3$, where $C$ is a smooth, connected, proper curve of genus $\geq 2$ over $k$. We assume that $C$ is simply ramified over $\mathbb{P}_{k}^{1}$, except possibly at one point $p \in \mathbb{P}_{h}^{1}$, 
where we allow arbitrary ramification. If $\varphi: C \rightarrow C$ is an automorphism such that $f \circ \varphi=f$, then $\varphi=\mathrm{id}$.

Proof. (cf. [13], p. 548 - the problem with Fulton's proof is that it apparently makes use of the characteristic zero assumption, so we trivially generalize his proof here for the sake of completeness). Let $\mu=H_{t}^{1}(\varphi)$ denote the induced map on $l$-adic cohomology, where $l$ is different from the characteristic of $k$. Since, as in well-known the automorphism group of $C$ is finite, we see that $\mu$ must have eigenvalues that are roots of unity; hence $|\operatorname{Tr}(\mu)| \leq 2 g$ (under any embedding of an algebraic closure of $\mathbb{Q}$, into $\mathbb{C}$ ). Thus, by the Lefshetz fixed point theorem, the number of fixed points of $\varphi$ is $\leq 2 g+2$. But since $\varphi$ must fix the $\geq(2 g-2)+2 d+1-(d-1)=2 g+d \geq 2 g+3$ branch points, we have a contradiction unless $\varphi=\mathrm{id}$.

Let us note that if $T^{\prime}$ is another $b$-point stack with corresponding Hurwitz stack $H^{\prime}$, and if $T^{\prime} \rightarrow T$ is one of the arrows in Diagram $1.1 \mathrm{~A}$, then we have $H^{\prime}=H \times_{T} T^{\prime}$.

$\S 1.4$. We are now going to define generalizations of the Hurwitz stacks, namely, "degenerate Hurwitz stacks", which we shall use to make explicit the compactification of the original Hurwitz stacks. We start by defining "degenerate $b$-point stacks". The reason for the use of the descriptor "degenerate" will become clear once these Hurwitz-type stacks are defined. As usual, we have four types, of which the first three are schemes:

(1) $\mathscr{T H} \mathbb{C}_{\mathrm{b}}:$ We take $\mathscr{H} C_{\mathrm{b}}$.

(2) $\mathscr{L} \mathscr{C F}_{\mathrm{b}}$ : Let $\mathscr{S}^{\mathrm{b}-1}$ act on the first $b-1$ sections of $\mathscr{M} C_{\mathrm{b}}$. Then take the quotient of $\| C_{\mathrm{b}}$ by this action. Note that (1) and (2) can be defined also for $b=3$.

(3) $\mathscr{H} \mathscr{R} C_{\mathrm{b}}:$ We take $\mathscr{R} c_{\mathrm{b}}$, when $b \geq 4$. When $b=3$, we take $\mathscr{R} C_{3}=\operatorname{Spec} R$.

(4) $\mathscr{Z} \mathscr{R} \mathscr{S}_{\mathrm{b}}$ : As before $\mathscr{J}^{\mathrm{b}-1}$ acts on $\mathscr{H} \mathrm{C}_{\mathrm{b}}$. Take the quotient by this action in the sense of stacks if $b \geq 4$. When $b=3$, take $\mathscr{K S}_{3}=\operatorname{Spec} R$.

As before, there is a functorial interpretation of these degenerate $b$-point stacks. We leave it to the reader to work out this interpretation in terms of various sorts of collections of sections of divisors in $\mathbb{P}^{1}$. In this interpretation, we shall call the first $b-1$ sections (resp. the étale divisor induced by the first $b-1$ sections) the marking sections (resp. marking divisor) and the last (i.e., $b^{\text {th }}$ ) section the clutching section. 
Now fix $\sigma \in \mathscr{S}^{\mathrm{d}}$. Let $T=$ one of the degenerate $b$-point stacks. Then we denote by $M \subseteq \mathbb{P}^{1} \times T$ (resp. $C \subseteq \mathbb{P}^{1} \times T$ ) the marking (resp. clutching) divisor. Let $D=M \cup C$. Then $M, C$, and $D$ are relative $T$-divisors with normal crossings. Moreover, $T$ is smooth over our original ring $R$. Now by [29], Exposé XIII, Appendice I, we can form $\mathscr{F}=\mathbb{R}_{e t}^{1} f_{\star} \mathscr{S}^{d}$, where $U=\left(\mathbb{P}^{1} \times T\right)-D, f: U \rightarrow T$ is the restriction of the projection to the second factor $\pi_{2}: \mathbb{P}^{1} \times T \rightarrow T$, and (by abuse of notation) $\mathscr{S}^{d}$ is the constant étale sheaf in noncommutative groups on $U$ with fibre $\mathscr{S}^{d}$ :

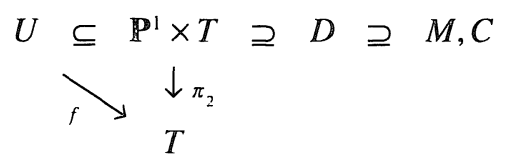

Thus $\mathscr{F}$ is a finite étale covering of $T$. Write $g: \mathscr{F} \rightarrow T$. For each geometric point $t$ of $T, g^{-1}(t)$ can be identified with $\operatorname{Hom}^{\prime}\left(\pi_{1}^{a l g}\left(\mathbb{P}_{t}^{1}-D_{t}\right), \mathcal{P}^{d}\right)$, where the "prime" after the Hom means "up to inner automorphism" (thus exempting us from the need to choose a base point for our $\pi_{1}$ ). Let us consider the closed and open substack $\mathscr{H} \subseteq \mathscr{F}$, which is also finite étale over $T$ and which is such that if $k=\left.g\right|_{\text {" }}$, then $k^{-1}(t)$ can be identified with those homomorphisms $\varphi: \pi_{1}^{\text {alg }}\left(\mathbb{P}_{t}^{1}-D_{t}\right) \rightarrow \mathscr{P}^{d}$ such that:

(1) $\varphi$ is surjective.

(2) $\varphi$ takes the generators of the monodromy groups around the marked points to transpositions.

(3) $\varphi$ takes the generator of the monodromy group around the clutching point to a conjugate of $\sigma$.

Then $\mathscr{K}$ will be our degenerate Hurwitz stack, which we shall denote, depending

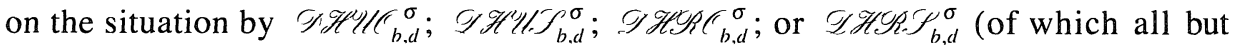
the last are schemes). Note that the degenerate Hurwitz stacks corresponding to conjugate $\sigma$ may be naturally identified.

§1.5. Remark. Unlike the original Hurwitz stacks, it is not clear (at least to the author) that these degenerate Hurwitz stacks should have any modular interpretation at all. Indeed, the logic of [13], §1.4, 1.5, does not follow through here because of the more complicated nature of the ramification involved. The problem is that if we denote by $\mathscr{F}$ what one might think is the appropriate modular stack (in terms of coverings of the projective line), and by $\mathscr{D} \mathscr{H}$ one of the degenerate Hurwitz stacks, we get morphisms $\alpha: \mathscr{F} \rightarrow \mathscr{Z} \mathscr{H}$ (clear) and $\beta: \mathscr{D} \mathscr{H} \rightarrow \mathscr{F}$ (by the "pseudo-universal" covering to be constructed in the following paragraph) and one can show that $\alpha \circ \beta=1_{r, \pi}$, hence that $\alpha$ is smooth. 
But in order to show that it is étale, one needs, in the case of simple coverings, the fact that the discriminant divisor is étale; since one does not have this fact here, one cannot follow through as before.

However, if $\mathscr{H} \mathscr{H}$ is one of the degenerate Hurwitz stacks, we can construct what one might call a "pseudo-universal" covering as follows. (Here we use the term "pseudo-universal" loosely in the sense that although $\mathscr{F} \mathscr{H}$ does not actually represent the moduli stack $\mathscr{F}$ of coverings of a certain type (in which case the tautological covering over $\mathscr{F} \mathscr{H}$ " would be called simply "universal"), at least over

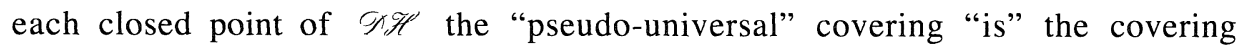
corresponding to that closed point). Indeed, it is tautological that there exists an étale covering $\psi:>\rightarrow\left(\mathbb{P}^{1} \times \mathscr{Y} \mathscr{H}\right)-D_{\text {, }}$, such that the normalization $\bar{\gamma}^{-} \rightarrow \mathbb{P}^{1} \times$ $\mathscr{M} \mathscr{H}$ of $\mathbb{P}^{1} \times \mathscr{M} \mathscr{H}$ in $\%$ is what we want. The only thing that is nonobvious is that $\bar{Y}$ is a smooth, proper curve over $\mathscr{M} \mathscr{H}^{\prime}$. But smoothness follows from the fact that $\mathscr{\mathscr { H }} \mathscr{H}$ is regular (since $T$ is) and by applying Abhyankar's lemma. Thus, to summarize we have the following diagram:

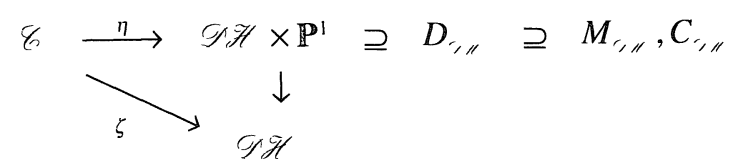

where $\forall=z^{-}, \zeta$ is smooth, proper, with geometrically connected fibres, $\eta$ is flat, étale outside of $D_{r_{\text {, }}}$, has simple ramification outside of $C_{\text {, / }}$, and has ramification of the type prescribed by $\sigma$ over $C_{1, "}$. In particular, by the Lemma of $\S 1.3$, it follows that if $d \geq 3$, then $\%$ has no automorphisms that fix $\eta$.

$\S 1.6$. Finally, let us note as before that we have diagrams:

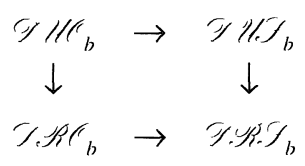

(Diagram 1.2A)

where the vertical arrows are $P G L(2)$-torsors, and the horizontal arrows are $\mathcal{J}^{b-1}$-torsors, and

$$
\begin{aligned}
& \pi_{1}^{t o p}\left(\mathscr{F} / / C_{b, C}\right) \rightarrow \pi_{1}^{t o p}\left(\mathscr{O} / H_{b, C}\right) \\
& \downarrow \quad \downarrow
\end{aligned}
$$

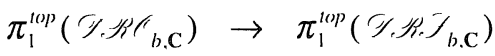

where the vertical arrows are surjections, and the horizontal arrows are injections. Note that if $\mathscr{D}^{\prime} \rightarrow \mathscr{D}$ is an arrow in the first diagram, then with respect to degenerate Hurwitz stacks and "pseudo-universal" curves, we have $\mathscr{Q} \mathscr{H}^{\prime}=\mathscr{D} \mathscr{H} \times, \mathscr{D}^{\prime}$ and $\mathscr{C}^{\prime}=\mathscr{C} \times{ }_{1, \|} \mathscr{D} \mathscr{H}^{\prime}$. 
Before continuing, we remark that although it is not absolutely necessary for what follows to use all four versions of the Hurwitz scheme, we presented them here in this introductory section in some detail so as to clarify which was which, since to the author's knowledge, there does not yet seem to exist either standard notation or standard terminology in the literature that allows one to specify precisely which Hurwitz scheme one is dealing with at any particular time.

\section{§2. Irreducibility}

§2.1. The first topological invariant that one wishes to compute about a newly constructed object is its connectedness, or irreducibility. Here we show that $\mathscr{H} \mathscr{W} \mathscr{S}_{b . d}$ and $\mathscr{D} \mathscr{C} \mathscr{W} \mathscr{S}_{b, d}^{\sigma}$ - which are schemes, so long as $d \geq 3$ - are geometrically irreducible over $R$. It then follows from Diagrams $1.1 \mathrm{~A}$ and $1.2 \mathrm{~A}$ that the stacks $\mathscr{H} \mathscr{R} \mathscr{S}_{b, d}$ and $\mathscr{D} \mathscr{Y} \mathscr{R} \mathscr{S}_{b, d}^{\sigma}$ are also geometrically irreducible over $R$. To prove irreducibility, we note that as in [13], pp. 546-7, we must show that the fundamental group of the appropriate $b$-point stack acts transitively on the set corresponding to the étale covering which is the Hurwitz scheme; as in loc. cit., we see that the fundamental group of the $b$-point stack has certain canonical elements that correspond to "braiding" various pairs of points; hence we are reduced to the following combinatorial Proposition.

\$2.2. Proposition. Fix $b, d \in \mathbb{N}, \sigma \in \mathscr{S}^{d}$. Let $A_{b, d}^{\sigma}$ be the set of ordered $b$ tuples of transpositions $\left\{t_{1}, \ldots, t_{b}\right\}$ of $\mathscr{S}^{d}$ such that $t_{1} t_{2} \ldots t_{b}=\sigma$ and such that the group generated by the $t_{i}$ 's acts transitively on $\{1, \ldots, d\}$. Define an "elementary move" on $A_{b, d}^{\sigma}$ as an automorphism of $A_{b, d}^{\sigma}$ that takes $\left\{t_{1}, \ldots, t_{t}, t_{t+1}, \ldots, t_{b}\right\}$ to $\left\{t_{1}, \ldots, t_{t+1}, t_{t+1} t_{t} t_{t+1}, \ldots, t_{b}\right\}$ for some $i$. Then the free group $F$ generated by the elementary moves acts transitively on $A_{b, d}^{\sigma}$.

(Psychological Remark. In the case of degenerate Hurwitz stacks, i.e. $\sigma \neq i d$, what we have called $b$ here really corresponds to $b-1$ in the previous section.)

Notational Remark. We shall denote chains of transpositions by $<?, ?, \ldots, ?>$, where each of the question marks is a transposition, and we shall make elementary moves on such chains, where we regard these chains as subchains of the original $b$-tuple of transpositions. It is clear that an elementary move on such a subchain corresponds to an elementary move on the original $b$-tuple. We call two elements $t, t^{\prime}$ of $A_{b . d}^{\sigma}$ equivalent if they lie in the same domain of transitivity of F. We write $t \sim t^{\prime}$.

Bibliographical Remark. It has been brought to the author's attention that similar results were obtained using similar techniques in the classical papers of [4], [20], and [25], as well as the more recent paper of [2]. 
§2.3. Lemma. We have the following relations:

(1) $<(12),(23)>\sim<(13),(12)>$

(2) <(12), (23), (23) > < (12), (13), (13) >

(3) $<(12),(13),(13)>\sim<(13),(13),(12)>$

(4) And lastly:

$$
\begin{aligned}
<(12),(12) & ,(23),(23),(23),(23)> \\
\sim & <(12),(12),(12),(12),(23),(23)>
\end{aligned}
$$

Proof. For (1) to (3), we have $<(12),(23)>\sim<(23),(13)>\sim<(13),(12)>$. Applying this twice, we get

$$
<(12),(23),(23)>\sim<(13),(13),(12)>
$$

On the other hand,

$$
<(12),(13),(13)>\sim<(13),(23),(13)>\sim<(13),(13),(12)>
$$

Putting this together, we obtain (1) through (3). As for (4), one sees by (3) that, up to equivalence, both sides of (4) are of the same form in the sense that they differ only in that the digits 1 and 3 have opposite roles on the two sides; moreover, a chain of this form may be uniquely specified by stating which digit (i.e., of 1,2, or 3) appears twice, which four times, and which six times. For the left hand side, these are, respectively, $(1,3,2)$; for the right hand side, they are $(3,1,2)$. Thus, if we think of the symmetric group on three letters acting on chains of this form by permuting the digits 1,2 , and 3 , we see that it suffices to show that the left hand side is equivalent both to " $(2,3,1)$ " and to " $(1,2,3)$ ". By the part of the lemma already proven, we have that the left hand side

$$
<(12),(12),(23),(23),(23),(23)>\sim<(12),(13),(13),(13),(13),(12)>=
$$

what we have referred to as " $(2,3,1)$ ". Since $\langle(12),(23)\rangle \sim<(23)$, (13) $>$, we have that the left hand side

$$
\begin{aligned}
<(12),(12),(23), & (23),(23),(23)> \\
\sim & <(23),(13),(13),(23),(23),(23)> \\
\sim & <(13),(13),(23),(23),(23),(23)>
\end{aligned}
$$


by (3). But this last chain is just " $(1,2,3)$ ".

\$2.4. Lemma。 Let $\alpha, \beta \in\{1, \ldots, d\}$ with $\alpha \neq \beta$. Let $t \in A_{b, d}^{\sigma}$. Then $t \sim t^{\prime}$ such that $t^{\prime}=(\alpha \beta)$.

Proof. Let us note first that by the transitivity assumption on elements of $A_{b, d}^{\sigma}$, we know that $\alpha$ and $\beta$ must appear somewhere as digits in the transpositions of $t$. By elementary moves, we can assume that $t_{1}$ contains $\alpha$; by subsequent elementary moves toward the front, and the transitivity assumption on elements of $A_{b, d}^{\sigma}$, we may assume that $t$ is of the following form:

$$
t_{1}=\left(\alpha x_{2}\right), t_{2}=\left(x_{2} x_{3}\right), t_{3}=\left(x_{3}, x_{4}\right), \ldots, t_{t}=\left(x_{t} x_{t+1}\right), \ldots, t,=(x, \beta), \text { with } r \leq b
$$

Here the $x_{t}$ 's are distinct from each other and from $\alpha$ and $\beta$. Now we have

$$
\left.\left\langle\left(\alpha x_{3}\right),\left(\alpha x_{2}\right)\right\rangle \sim<\left(\alpha x_{2}\right),\left(x_{2} x_{3}\right)\right\rangle=\left\langle t_{1}, t_{2}\right\rangle
$$

so by substituting $\left\langle\left(\alpha x_{3}\right),\left(\alpha x_{2}\right)\right\rangle$ for $\left\langle t_{1}, t_{2}\right\rangle$, moving the $t_{t}$ 's for $3 \leq i \leq r$ down one position to the left, and sending (what would originally have been) ( $\left.\alpha x_{2}\right)$ out to the right beyond what was $t_{r}$, we see that we obtain the same situation as what we started with, except that $r$ is one less than it was before. Now apply induction. o

\$2.5. Let us prove the Proposition first in the case of the nondegenerate Hurwitz stack, i.e., when $\sigma=\mathrm{id}$. Let $t \in A_{b, d}^{(1 d)}$. We shall show that $t \sim$ a chain which consists of a nonzero, even number of (12)'s, followed by a nonzero, even number of (23)'s, and so on through $(d-1, d)$. By Lemma 3, (4), all such chains are equivalent, so we are done.

By transitivity, Lemma 4 allows us to assume that $t_{1}=(12), t_{2}=(12)$ on account of the fact that $\sigma=\mathrm{id}$. By using inverse elementary moves to bring things to the front, we may assume that $t_{3}, \ldots, t$, contain a " 1 ", but that $t_{i}(\forall i>r)$ do not. We claim that we may move to a situation where (with possibly a new $r$ ) $t_{3}, \ldots, t$, are all equal to (12). For, if there is an $i$ such that $3 \leq i \leq r-1$ and $t_{t}=(1 a)$, $t_{t+1}=(1 b)$ with $1 \neq a \neq b$, then $\langle(1 a),(1 b)\rangle \sim\langle(1 b),(a b)\rangle$, so we can reduce to the same situation with lower $r$. Thus we may assume that $t_{3}=t_{4}=\ldots=t$, If $t_{3}=(12)$, then we have proven the claim. If $t_{3}=(1 a)$ with $a \neq 2$, then it is easy to see that $r$ must be even (since $t_{t}(\forall i>r)$ doesn't contain a " 1 "); hence, applying Lemma $3,(2)$, proves the claim. Now repeating the above procedure finishes the proof for $\sigma=\mathrm{id}$. 


\section{\$2.6. Proof of Proposition 2.}

Step A. First we introduce some notation. Write $\sigma=\gamma_{1} \ldots \gamma_{r}$ where each

$$
\gamma_{1}=\left(a_{1}^{(l)}, a_{2}^{(l)}, \ldots, a_{e_{t}}^{(l)}\right)
$$

is a nontrivial cycle with domain of transitivity $\Delta_{l}$ such that $\Delta_{l} \cap \Delta_{1}=\emptyset$ for $i \neq j$. Let $q=r+d-\left(\sum_{t=1}^{\prime} e_{t}\right), N=\left(\sum_{t=1}^{\prime} e_{t}\right)-r$. Then let the $\Delta_{t}$ for $r+1 \leq i \leq q$ be the remaining trivial domains of transitivity of $\sigma$. For simplicity, let's write $1_{1}$, 2, etc. for $a_{1}^{(l)}, a_{2}^{(l)}, \ldots$ Now define

$$
\mathscr{Z}_{l}=<(1,2,),\left(1,3_{1}\right),(1,4,), \ldots,\left(1,\left(e_{1}\right)_{l}\right)>\in A_{\left(e_{1}-1\right), e_{l}}^{\gamma_{1}}
$$

Let.$\psi^{2}$ denote the concatenation of chains $\mathscr{Z}_{1}, \mathscr{Z}_{2}, \ldots, \mathscr{Z}_{1}$.

Now let $t \in A_{b, d}^{\sigma}$. By Lemma 4, we see that we may assume that $t$ begins with $A^{\prime}$. (Note that this same logic already proves the Proposition for $A_{\left(e_{t}-1\right), e_{t}}^{\gamma_{t}}$. ) Thus we have normalized $t_{1}, \ldots, t_{N}$. It remains to deal with $t_{\imath}$ for $i>N$.

Step B. Let $\mathscr{D}_{1}, \ldots, \mathscr{D}_{3}(\subseteq\{1, \ldots, d\})$ be the domains of transitivity of the group generated by the $t$, for $i>N$, (so $\bigcup_{t=1}^{\prime} \mathscr{D}_{1}=\{1, \ldots, d\}$ and $\mathscr{D}_{1} \cap \mathscr{D}_{J}=\varnothing \forall i \neq j$ ). For $1 \leq j \leq s$, let

$$
\mathscr{T}_{1}=\left\{i \in\{1, \ldots, b\} \mid i>N \text { and } t_{1} \text { acts nontrivially on } \mathscr{D}_{1}\right\}
$$

Note that if we take the subchain $\tau^{J}$ of transpositions of $t$ indexed by $\mathscr{P}$, then $\tau^{\jmath} \in A_{\mid\langle,|,|>,|}^{(d d)}$. By $\$ 2.5$, we may assume that $\tau^{\prime}$ consists of a sequence of pairs of identical transpositions. Now we claim:

Claim. If $q \neq 1$. then we may assume that every $t_{t}(\forall i>N)$ is of the form (xy) with $x \in \Delta_{k}, y \in \Delta_{l}, k \neq l$.

Proof of Claim. Suppose we have a pair $t_{t}=t_{t+1}=(a b)$ where $i \equiv N+1(\bmod 2)$ and $a, b \in \Delta_{k}$ with $a \neq b$. Since all the $t_{t}$ 's together generate a group transitive on $\{1, \ldots, d\}$, there exists a $j>N$ with $j \equiv N+1(\bmod 2)$ such that $t_{J}=t_{j+1}=(c d)$ with $c \in \Delta_{k}$ and $d \in \Delta_{l}$ where $l \neq k$. We may move $t_{t}, t_{t+1}$ so that they are adjacent to $t_{1}, t_{\jmath+1}$ (by Lemma $3,(3)$ ), so we obtain:

$$
<(c d),(c d),(a b),(a b)>
$$

By Lemma 7A below, we may assume that $b=c$. Then applying Lemma 3, (2), with $1,2,3$ replaced by $d, c, a$, respectively, we have 


$$
<(c d),(c d),(a c),(a c)>\sim<(c d),(c d),(a d),(a d)>
$$

Repeating this procedure proves the claim. $O$

\$2.7. Lemma $\mathbb{A}$. Suppose in the above situation (except that $q$ might be one) that we have a pair $t_{t}=t_{t+1}(a b)$ with $i \equiv N+1(\bmod 2)$, and $a, b \in \Delta_{k}, a \neq b$. Then we may move to the same situation, except with $a=1_{k}, b=2_{k}$.

Proof. We may assume that with respect to the ordering $1_{k}<2_{k}<3_{k}<\ldots$, we have $a<b$. By moving around chunks of $t$ that commute with each other, we may assume that $i=N+1$ and we may move $\mathscr{Z}_{k}$ right up to the left of $t_{N+1}$ :

$$
\begin{gathered}
t=\mathscr{Z}_{1} \mathscr{Z}_{2} \ldots \mathscr{Z}_{k} \ldots \mathscr{Z}_{r} t_{N+1} t_{N+2} \ldots t_{b} \\
\downarrow \downarrow \text { goes to } \\
t=\mathscr{Z}_{1} \mathscr{Z}_{2} \ldots \mathscr{Z}_{h-1} \mathscr{Z}_{k+1} \ldots \mathscr{Z}_{1} \mathscr{Z}_{k} t_{N+1} t_{N+2} \ldots t_{b}
\end{gathered}
$$

Suppose $a \neq 1_{h}$. Since we have shown the Proposition for $A_{\left(e_{h}-1\right), e_{h}}^{\gamma_{h}}$, we may assume that $\mathscr{Z}_{k}$ ends with the transposition $((a-1), a)$. By Lemma 3, (2),

$$
<((a-1), a),(a b),(a b)>\sim<((a-1), a),((a-1), b),((a-1), b)>
$$

By repeating this procedure many times, we can assume that $a=1_{h}$. Applying the same process to $b$ proves the Lemma. $O$

Lemma $\mathbb{B}$. In the above situation, suppose that we have a pair $t_{t}=t_{t+1}=$ (ab) (where $i \equiv N+1(\bmod 2)$ ) with $a \in \Delta_{k}$ and $b \in \Delta_{l}$, where $k \neq l$. Then we may assume that $a=1_{k}, b=1_{l}$.

Proof. Same as for Lemma A. O

$\S 2.8$. By Lemma 7B and the claim at the end of $\S 2.6$, if $q \neq 1$, then we may assume that every $t_{t}$ (for $i>N$ ) is of the form $\left(1_{k} 1_{l}\right)$ for $k \neq l$. If $q=1$, then $\Delta_{1}=\{1, \ldots, d\}$ and we may assume that every $t_{\imath}$ (for $i>N$ ) is of the form $\left(1_{1} 2_{1}\right)$. Thus if $q=1$, we have found a standard normalizing form for $t$, so the Proposition has been proved.

If $q \neq 1$, then we note that if we regard $\left(1_{k} 1_{l}\right)$ as an element of the permutation group of the symbols $\Delta_{1}, \ldots, \Delta_{q}$, then the $t_{1}$ 's (for $i>N$ ) act transitively on the $\Delta_{j}$ 's (since all the $t_{t}$ 's together act transitively on $\{1, \ldots, d\}$ ); on the other hand, $t_{N+1} t_{N+2} \ldots t_{b}=(i d)$. Thus we are in the situation of dealing with an element of $A_{(b-N), q}^{(1 d)}$. But the Proposition has already been proven in this case. Thus we are done. $\mathrm{O}$ 
$\S 2.9$. For ease of reference, we restate the main result of this section as a Theorem:

Theorem. For all $b, d \in \mathbb{N}$ such that $b>3$, and all $\sigma \in \mathscr{S}^{d}$, we have that

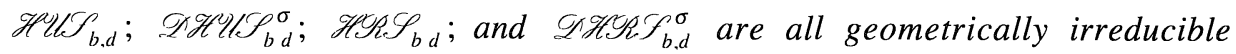
over $R$.

$\S 2.10$. We now turn to the proof of another similar irreducibility result which will be useful later. Let us recall the situation of $\S 1.5$ :

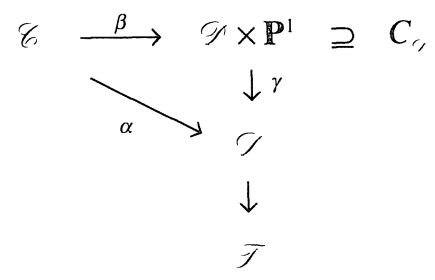

(Here $\mathcal{T}$ is the relevant degenerate $b$-pointed stack.) By $\S 4.3$, we see that the reduced inverse image $\mathscr{E}$ of $C_{r}$, via $\beta$ is étale over $C_{r} \cong \mathscr{Z}$. (We placed this Lemma in $\$ 4$ in which it is more in context, since this Section is concerned primarily with combinatorics.) Thus $\mathscr{E} \rightarrow \mathscr{T}$ is an étale covering. It is clear that the portions of $\mathscr{E}$ that correspond to different ramification indices form different connected components, even over $R$. In fact, we have the following result:

Proposition. The geometric (i.e., after tensoring from $R$ up to $\mathbf{C}$ ) connected components of $\mathscr{E}$ are in one-to-one correspondence with the set of ramification indices over $C_{1,}$, which is the same as the set of cardinalities of domains of transitivity of $\sigma$, where $\mathscr{T}=\mathscr{F} \mathscr{H} \mathcal{S}_{b, d}^{\sigma}$ or $\mathscr{B} \mathscr{H} \mathscr{R} \mathcal{S}_{b, d}^{\sigma}$.

Proof. As before, we consider the action of braiding elements of the fundamental group of $\bar{F}$ on the set $B$ corresponding to the étale covering $\because \rightarrow \bar{F}$. Simple reflection reveals that $B$ may be given the following description: $B$ consists of the set of pairs $\left\{\left(t_{1}, \ldots, t_{b-1}\right), \Delta\right\}$ where $\left(t_{1}, \ldots, t_{b-1}\right) \in A_{b-1, d}^{\tau}$, with $\tau$ conjugate to $\sigma$ and $\Delta$ a domain of transitivity of $\tau$.

The action of braiding on the first $b-1$ factors clearly doesn't affect $\tau$, and acts via an "elementary move" on the $A_{b-1, d}^{\tau}$ part and trivially on $\Delta$. The action of double braiding between the last two factors clearly takes the domain of transitivity $\Delta$ of $\tau$ to the domain of transitivity $t_{b-1}(\Delta)$ of $t_{b-1} \tau t_{b-1}$. By Proposition 2 , we can take any given element of $B$ to an element with $t_{b-1}$ arbitrary via braiding in the first $b-1$ factors. By doing this and then double braiding in the last two factors, it is thus clear that we can take any domain of transitivity $\Delta$ of $\tau$ to 
any other domain of transitivity of $\tau$ with the same cardinality. This completes the proof. $O$

\section{§3. Log Admissible Coveroings}

$\S 3.1$. In this paper, we will be interested in studying not only the various Hurwitz stacks introduced in $\S 1$, but also their compactifications. In [19], a definition of an "admissible covering" is proposed as a means of compactifying the Hurwitz scheme $\mathscr{H} \mathscr{R} c_{b, d}$. Unfortunately, since Harris-Mumford are interested only in the coarse moduli scheme of such admissible coverings, their definition of an admissible covering is not well suited to the study of families and the construction of an algebraic stack of admissible coverings. The main reason for this is that the natural context in which to study such admissible coverings is not the category of schemes, but rather that of log schemes, as defined by Kato in [21]. Thus, in this Section, we propose to give a foundational treatment of $\log$ admissible coverings. In particular, we prove a rather general existence theorem for a log algebraic stack of such coverings. If we forget the log structure of this log algebraic stack, and consider the associated coarse moduli space (as in [11], Theorem 4.10), we recover the coarse moduli scheme of Harris-Mumford.

Remark. In an earlier version of this paper, we referred to the proof of [19] in our construction of a moduli stack of admissible coverings. Indeed, the existence of such an algebraic moduli stack is essentially a formal consequence of what [19] claim to have proven. However, various people who read that earlier version complained bitterly that they could not follow the proof in [19]. Upon closer inspection, the author discovered a counterexample to one of the key claims in the proof of the existence of a coarse moduli scheme in [19] (see §3D below for more details). Thus, it seems that at this point, one must consider the proof of this result in [19] to be incomplete. In light of this situation, the author decided that it would be best to give a detailed treatment of the existence of an algebraic moduli stack of admissible coverings that is independent of what is done in [19].

\section{$\S 3 A$ A B asic Definitions}

$\S 3.2$. We start by reviewing Knudsen's notion of an $r$-pointed stable curve. Let $S$ be a scheme.

Defimition。A flat, proper morphism $f: C \rightarrow S$ of schemes together with $r$ sections $\sigma_{1}, \ldots, \sigma,: S \rightarrow C$ is called an r-pointed stable curve of genus $g$ if 
(1) the geometric fibres $C_{\bar{s}}$ off are connected, reduced, of dimension 1, have $\operatorname{dim}_{k(s)} H^{1}\left(C_{\bar{s}}, O_{C_{\bar{s}}}\right)=g$, and have at most ordinary double points as singularities;

(2) the images of the sections $\sigma_{1}, \ldots, \sigma$, are disjoint and land in the smooth locus of $f$;

(3) the relative dualizing sheaf $\omega_{C / S}$ is relatively ample with respect to $f$.

We shall refer to the sections $\sigma_{1}, \ldots, \sigma_{r}$ as the marked points of $f: C \rightarrow S$, and to the divisor in $C$ defined by the union of the $\sigma_{1}$ as the divisor of marked points.

Note that the ampleness condition (3) implies that $2 g-2+r \geq 1$. [22] shows that there exists a smooth, proper (over $\mathbb{Z}$ ) moduli stack for such data, which we shall denote by $\overline{\mathscr{T}}_{g . l}$. Those $r$-pointed stable curves for which $C \rightarrow S$ is smooth define an open substack $\mathscr{M}_{g, l} \subset \bar{M}_{g, l}$. The complement of $\mathscr{M}_{g, r}$ in $\overline{\mathscr{M}}_{g, l}$ is a divisor with normal crossings. We shall call this divisor the divisor at infinity of $\overline{\mathbb{M}}_{g . r}$.

We shall often find it convenient to use the language of log structures and log schemes of [21]. A $\log$ structure on $S$ consists of an étale monoid $M$ on $S$, together with a morphism $\mathscr{M} \rightarrow C_{S}$ of monoids that satisfies certain properties. See [21] for more details. We shall write the monoid operation on sections of $M$ additively, and refer to the given morphism $\mathscr{I l} \rightarrow C_{S}$ as the exponential map of the log structure.

The divisor at infinity of $\overline{\mathbb{M}}_{g .,}$ thus defines (as in [21], $\$ 1.5,(1)$ ) a $\log$ structure on $\overline{\mathscr{K}}_{g, l}$ which we shall call the canonical log structure on $\overline{\mathscr{M}}_{g, l}$. We

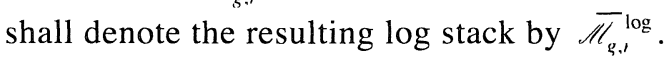

Let $M_{\bar{I}_{p},}$ denote the étale monoid that defines the canonical log structure on $\bar{M}_{g, l}$. Thus $M_{\bar{M}_{p},}$ is precisely the subsheaf of ${ }^{\prime \prime} \bar{"}_{\varphi,}$ consisting of functions invertible on $\mathscr{M}_{g, l}^{p}$. Let

$$
G_{\overline{\pi_{0}}} \subseteq M_{\overline{\bar{u}_{1}}}
$$

be the subsheaf of functions whose zero locus is smooth (over $\mathbf{Z}$ ). Thus,

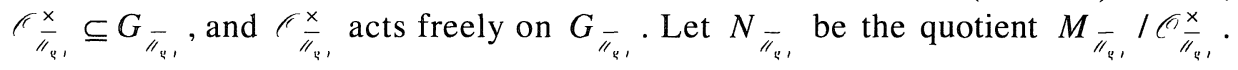
Then

$$
\Gamma_{\bar{\pi}_{p},} \stackrel{\text { det }}{=} G_{\bar{\pi}_{p}} / C_{\bar{\pi}_{p}}^{\times} \subseteq N_{\bar{\pi}_{p}}
$$


is a constructible sheaf of sets (in the étale topology). Finally, note that $G_{\bar{\pi}_{0} \text {, }}$ generates the sheaf of monoids $M_{\overline{\pi_{p}}}$.

Let $\left(\mathscr{C} \rightarrow \overline{\mathscr{M}}_{g, j} ; \sigma_{1}, \ldots, \sigma,: \overline{\mathscr{M}}_{g, l} \rightarrow \mathscr{C}\right)$ denote the universal $r$-pointed stable curve of genus $g$. It is shown in [22] that $\mathscr{C}$ may be naturally identified with $\overline{\mathscr{M}}_{g, r+1}$. Let us denote by $\mathscr{C}^{\log }$ the log stack obtained by pulling back the canonical $\log$ structure on $\overline{\mathscr{M}}_{g, r+1}$ relative to this identification. We shall call this log structure on $\mathscr{E}$ the canonical log structure on $\mathscr{E}$. Note that the canonical log structure on $\mathscr{E}$ can also be obtained as the log structure associated to the divisor with normal crossings which is the union of the images of the $\sigma_{1}$, together with the pull-back via $\mathscr{C} \rightarrow \overline{\mathbb{M}}_{\text {g,r }}$ of the divisor at infinity of $\overline{\mathscr{M}}_{g, l}$. Thus, just as for $\overline{\mathscr{M}}_{g, l}$, we can define $G, \subseteq M$, and $\Gamma, \stackrel{\text { def }}{=} G, I \mathcal{C}^{\times} \subseteq N, \subseteq$

§3.3. When working in the logarithmic category, the following notion is more convenient than the classical notion of Knudsen reviewed above. Let $S^{\text {log }}$ be a fine log scheme (in the sense of [21], §2.3).

Definition.A morphism of log stacks $\kappa^{\log }: S^{\log } \rightarrow \overline{\mathscr{M}}_{g . r}^{\log }$ will be referred to as the data for an r-pointed stable log-curve of genus $g$. For such $a \kappa^{\log }$, let $C^{\log } \rightarrow S^{\log }$ be the pull-back (in the logarithmic category) of the universal object $\mathscr{C}^{\log } \rightarrow \overline{\mathscr{M}}_{g . r}^{\log }$ by means of $\kappa^{\log }$. Such a $C^{\log } \rightarrow S^{\log }$ will be referred to as an $\mathrm{r}$ pointed stable log-curve of genus $g$.

Note that for smooth curves, once one is given a classifying morphism $\kappa: S \rightarrow \overline{\mathscr{M}}_{g, l}$, the resulting $\log$ morphism $\kappa^{\log }: S^{\log } \rightarrow \overline{\mathscr{M}}_{g, l}^{\log }$ is uniquely defined, so smooth curves and smooth log-curves are equivalent notions. However, if $\kappa: S \rightarrow \overline{\mathscr{M}}_{g, l}$ does not map into $\mathscr{M}_{g, l}$, then it is easy to construct examples of distinct $\log$ morphisms $S^{\log } \rightarrow \overline{\mathscr{M}}_{g, \mathrm{l}}^{\log }$ with the same underlying stack morphism $\kappa: S \rightarrow \overline{\mathscr{M}}_{g, l}$. Nonetheless, given a morphism $\kappa: S \rightarrow \overline{\mathscr{M}}_{g, r}$, there always exists a natural choice of $\log$ structure on $S$ for which $\kappa$ extends to a logarithmic morphism: namely, the pull-back of the log structure on $\overline{\mathscr{M}}_{g, 1}^{\log }$.

Now let $C^{\log } \rightarrow S^{\log }$ be an $r$-pointed stable log-curve of genus $g$. Let $M_{C}$ (respectively, $M_{S}$ ) denote the étale monoid defining the log structure of $C^{\text {log }}$ (respectively, $S^{\log }$ ). Let $N_{C}$ (respectively, $N_{S}$ ) be the quotient $M_{C} / \sigma_{C} \times$ (respectively, $\left.M_{S} / C_{S}{ }^{x}\right)$. Then we obtain a natural morphism form $\Gamma_{r} l_{C}$ (respectively, $\Gamma_{\bar{I}_{a}}, l_{S}$ ), to $N_{C}$ (respectively, $N_{S}$ ). We denote the image of this morphism by $\Gamma_{C}$ (respectively, $\Gamma_{S}$ ). Thus, $\Gamma_{C}$ (respectively, $\Gamma_{S}$ ) forms a subsheaf (of sets) of $N_{C}$ (respectively, $N_{S}$ ). Let $G_{C}$ (respectively, $G_{S}$ ) be the 
subsheaf (of sets) of $N_{C}$ (respectively, $N_{S}$ ). Let $G_{C}$ (respectively, $G_{S}$ ) be the inverse image of $\Gamma_{C}$ (respectively, $\Gamma_{S}$ ) in $M_{C}$ (respectively, $M_{S}$ ) via the projection $M_{C} \rightarrow N_{C}$ (respectively, $M_{S} \rightarrow N_{S}$ ). We shall call $\Gamma_{C}$ (respectively, $\Gamma_{S} ; G_{C} ; G_{S}$ ) the sheaf of generators of $N_{C}$ (respectively, $N_{S} ; M_{C} ; M_{S}$ ). Since $\Gamma_{C}$ is a constructible sheaf of sets on $C$, one can regard (see [28], p. 160) $\Gamma_{C}$ as a quasi-finite algebraic space over $S$. Then $G_{C}$ defines an $\mathscr{C}_{\Gamma_{C}}^{\times}$-torsor, hence a line bundle $\mathscr{L}_{C}$ on this algebraic space $\Gamma_{C}$.

Finally, by forgetting log structures, we obtain an underlying r-pointed stable curve of genus $g:\left(f: C \rightarrow S ; \sigma_{1}, \ldots, \sigma_{r}: S \rightarrow C\right)$.

§3.4. Sometimes it is convenient to forget the ordering on the marked points of a pointed stable curve. That is to say, we have a natural action of $\mathscr{S}^{\prime}$ (the symmetric group on $r$ letters) on $\overline{\mathscr{M}}_{g, l}^{\log }$ given by permuting the marked points. If we form the quotient of $\overline{\mathscr{M}}_{g, l}{ }^{\log }$ by $\mathscr{S}^{\prime}$ (in the sense of stacks), we thus obtain a log stack $\overline{\mathscr{M}}_{g, l}^{\log }$. Note that the universal log-curve $\mathscr{E}^{\log } \rightarrow \overline{\mathscr{M}}_{g, 1}^{\log }$ descends to a $\log$-curve $\mathscr{E} \mathscr{S}^{\log } \rightarrow \overline{\mathscr{N}}_{g, 1}^{\log }$. Let $S^{\log }$ be a fine log scheme.

Definition. A morphism of log stacks $\kappa^{\log }: \mathscr{S}^{\log } \rightarrow \overline{\mathscr{M S}}_{\mathrm{g} . \mathrm{l}}^{\log }$ will be referred to as the data for a symmetrically $r$-pointed stable log-curve of genus $g$. For such a $\kappa^{\log }$, let $C^{\log } \rightarrow S^{\log }$ be the pull-back (in the logarithmic category) of the universal object $\mathscr{E S}^{\log } \rightarrow \overline{\mathscr{H}}_{\mathcal{F}_{\mathrm{g}, 1}}^{\log }$ by means of $\kappa^{\log }$. Such a $C^{\log } \rightarrow S^{\log }$ will be referred to as a symmetrically r-pointed stable log-curve of genus $g$. If we forget the log structures of such a log-curve, the resulting $\left(f: C \rightarrow S ; \mu_{f} \subseteq C\right.$ ) (where $\mu_{f} \subseteq C$ is the divisor of marked points) will be referred to as a symmetrically rpointed stable curve of genus $g$.

For instance, when $g=0$, the open substack $\mathscr{M S}_{g, l} \subseteq \overline{\mathbb{M S}}_{g, l}$ of smooth curves may be identified with the stack $\mathscr{R}_{b}$ of $\$ 1.3$.

$\S 3.5$. We are now ready to state the main definition of this Section. Let $f^{\log }: C^{\log }$ $\rightarrow S^{\log }$ (respectively, $h^{\log }: D^{\log } \rightarrow S^{\log }$ ) be a symmetrized $r$-(respectively, $s^{-}$) pointed stable log-curve of genus $g$ (respectively, $q$ ). Let $\mu_{f} \subseteq C$ (respectively, $\mu_{h} \subseteq D$ ) be the divisor of marked points. Let $\pi^{\log }: C^{\log } \rightarrow D^{\log }$ be a morphism of $\log$ schemes.

Definition. We shall say that $\pi^{\log }: C^{\log } \rightarrow D^{\log }$ is a log admissible covering of degree $d$ if the following conditions are satisfied:

(1) $\pi^{\log }$ is log étale (in the sense of [21], §3.3); moreover, the morphism $\pi^{-1} N_{D} \rightarrow N_{C}$ induced by the morphism of log structures maps $\Gamma_{D}$ into 


$$
\Gamma_{C}^{d} \stackrel{\text { def }}{=} \bigcup_{i=1}^{d} i \cdot \Gamma_{C}
$$

(2) $\pi$ is finite;

(3) $\pi$ is of degree $d$ on a dense open set of every fiber of $h: D \rightarrow S$;

(4) $\mu_{f} \subseteq \pi^{-1}\left(\mu_{h}\right) ; \pi^{-1}\left(\mu_{h}\right) \subseteq d \cdot \mu_{f}$;

(5) $f$ is smooth at $c \in C$ if and only if h is smooth at $\pi(c)$.

We now make some trivial observations concerning log admissible coverings:

(1) Of the above conditions, only the first depends on the log structures; the rest depend only on the morphism of schemes $\pi$.

(2) Note that the second inclusion in condition (4) actually follows from conditions (1) and (5).

(3) Let $x \in C-\mu_{f}$ be a point at which $f$ is smooth. Let $y=\pi(x)$. Then the $\log$ structure on $C^{\log }$ (respectively, $D^{\log }$ ) at $x$ (respectively, $y$ ) is just the pull-back to $C$ (respectively, $D$ ) of the $\log$ structure on $S^{\log }$. It thus follows that $\pi$ is étale at $x$.

(4) If $h^{\log }$ has the property that every irreducible component of every geometric fiber $D_{\bar{s}}$ of $h$ is smooth, then $f^{\text {log }}$ also has this property.

§3.6. Suppose that now that $\left(f: C \rightarrow S, \mu_{f} \subseteq C\right)$ and $\left(h: D \rightarrow S, \mu_{h} \subseteq D\right)$ are symmetrically pointed stable curves. Then we make the following definition

Definition. A morphism $\pi: C \rightarrow D$ will be called an admissible covering if there exist, étale locally on $S$ :

(1) a fine log scheme $S^{\log }$ with underlying scheme $S$;

(2) symmetrically pointed stable log-curves $C^{\log } \rightarrow S^{\log }$ and $D^{\log } \rightarrow S^{\log }$ whose underlying symmetrically pointed stable curves are $\left(f: C \rightarrow S, \mu_{f} \subseteq C\right)$ and $\left(h: D \rightarrow S, \mu_{h} \subseteq D\right)$;

(3) a log admissible covering $\pi^{\log }: C^{\log } \rightarrow D^{\log }$ whose underlying scheme morphism is $\pi$ 
Remark. We shall see below that when $D \rightarrow S$ is of genus zero, the definition agrees with that of [19], p. 57. The reason that we prefer this definition is that when considering moduli of morphisms of curves, it is very difficult to incorporate conditions that involve the existence of certain local functions on the curves (as do the conditions given in [19], p. 57). Since a log structure essentially involves some sort of complicated monoid-worth of global (on the curve) line bundles, a morphism of log structures amounts to some sort of morphism of line bundles, hence (as we shall see below) is relatively easy to consider the moduli of. On the other hand, one draw-back of the above definition of an admissible covering is that the $\log$ structure on $S$ is not uniquely or canonically defined. Nonetheless, we shall see below a posteriori that, in fact, there does always exist a canonical $\log$ structure on $S$ with respect to which an admissible covering $\pi$ extends to a log admissible covering.

\section{$\S 3 \mathbb{B}$. Finst Properties}

$\S 3.7$. Let $A$ be a local noetherian henselian ring. Let $s \in m_{A}$ (the maximal ideal of $A$ ). Let $R$ be the henselization of $A[X, Y] /(X Y-s$ ) (where $X$ and $Y$ are indeterminates) at the ideal generated by $m_{A}, X$, and $Y$. Thus, $R$ is a local noetherian henselian ring, with maximal idea $m_{R}$. Let $x, y \in R$ be the images of $X$ and $Y$, respectively. Then we have the following

Lemma. Suppose that we are given $x^{\prime}, y^{\prime} \in R ; s^{\prime} \in A$ such that $x^{\prime} y^{\prime}=s^{\prime}$, and $\left(x, y, m_{A}\right)=\left(x^{\prime}, y^{\prime}, m_{A}\right)$ (equality of ideals in $\left.R\right)$. Then there exist units $u_{1}, u_{1} \in R^{\times}$with $u_{1} u_{1} \in A^{\times}$such that either $u_{1} x=x^{\prime}$ and $u_{1} y=y^{\prime}$, or $u_{1} x=y^{\prime}$ and $u_{\imath} y=x^{\prime}$.

Proof. Let us first consider the case when $s=0$. Then the Lemma follows immediately from the simple structure of the ring $A[[X, Y]] /(X Y)$, and faithfully flat descent from $A[[X, Y]] /(X Y)$ down to $R$. Now let us return to the case of arbitrary $s$. By considering the ring $R / s R=A / s A[[X, Y]] /(X Y)$ (and applying the Lemma to this ring), it thus follows that $s^{\prime} \in(s)$ Moreover, without loss of generality, we may assume that $x^{\prime}=x+s \cdot \phi$ and $y^{\prime}=y+s \cdot \psi$, for some $\phi, \psi \in R$. By multiplying $x^{\prime}$ and $y^{\prime}$, we thus obtain that $s^{\prime}$ is equal to $s$ times a unit in $R$. Since $R$ is faithfully flat over $A$, it follows that $s^{\prime}$ is equal to $s$ times a unit in $A$. Thus, without loss of generality, we may assume that $s^{\prime}=s$. Let $R^{\prime}$ (respectively, $A^{\prime}$ ) be the completion of $R$ of (respectively, $A$ ). If we restrict ourselves to finding $u_{1}$, and $u_{1}$ such that $u_{1} u_{1}=1$, then it follows from the definition of "henselization," that it suffices to prove the Lemma with $R$ and $A$ replaced by $R^{\prime}$ and $A^{\prime}$, respectively. 
Let us review our situation: We have $x^{\prime}, y^{\prime} \in R^{\prime}$ such that $x^{\prime} y^{\prime}=s$ and $\left(x^{\prime}, y^{\prime}, m_{A^{\prime}}\right)=\left(x, y, m_{A^{\prime}}\right)$. Suppose we know that $x^{\prime}=x+s^{\prime} f ; y^{\prime}=y+s^{\prime} g$, for some $f, g \in R^{\prime}$, and some positive integer $i$. By multiplying $x^{\prime}$ and $y^{\prime}$, we obtain that $x\left(s^{l} g\right)+y\left(s^{l} f\right) \in s^{t+1} R^{\prime}$. Thus, $x+y$ annihilates the image of $x\left(s^{\prime} g\right) \equiv-y\left(s^{\prime} f\right)$ in $s^{\prime} R^{\prime} / s^{t+1} R^{\prime}$. Since $x+y$ is a regular element of $R^{\prime}$, we thus obtain that $x s^{\prime} g \equiv y s^{\prime} f \equiv 0 \in\left(s^{t+1}\right)$. On the other hand, from the structure of the module $s^{\prime} R^{\prime} / s^{l+1} R^{\prime}=s^{\prime} A^{\prime} / s^{l+1} A^{\prime}[[X, Y]] /(X Y)$, one then obtains that $s^{l} f-s^{\prime} x \alpha, s^{\prime} g-s^{\prime} y \beta$ $\in\left(s^{\prime+1}\right)$, for some $\alpha, \beta \in R^{\prime}$ such that $\alpha$ (respectively, $\beta$ ) is a power series in $x$ (respectively, $y$ ). Now let us note that we can always make substitutions of the following sort: replace $x^{\prime}$ by $x^{\prime} \cdot u^{-1}$, and $y^{\prime}$ by $y^{\prime} \cdot u$ (for some unit $u \in\left(R^{\prime}\right)^{x}$ ), without affecting the validity of the Lemma. Moreover, if we make such a substitution, with $u=1+s^{\prime} \alpha-s^{\prime} \beta$, we find ourselves in the situation reviewed at the beginning of this paragraph, except with $i$ replaced by $i+1$. By induction (and the fact that $R^{\prime}$ is complete and separated in the $s$-adic topology), this completes the proof of the Lemma. $O$

$\S 3.8$. We continue with the notation of the previous $\S$.

Lemma. Suppose that there exist units $u_{1}, u_{1} \in R^{\times}$such that $x u_{1}=x$, $y u_{v}=y$, and $u_{\imath} u_{v} \in A^{\times}$. Then it follows that $u_{\imath}=u_{\uparrow}=1$.

Proof. As in the previous $\S$, we prove the Lemma with $R$ and $A$ replaced by their completions $R^{\prime}$ and $A^{\prime}$. Suppose that we know that $u_{1} \equiv 1, u_{v} \equiv 1$ modulo $s^{\prime} R^{\prime}$, for some nonnegative integer $i$. Let $d_{1}=1-u_{1} ; d_{v}=1-u_{v}$. Since $x d_{1}=0$, $y d_{v}=0$, it follows that we can write $d_{\imath}\left(\bmod s^{t+1} R^{\prime}\right)\left(\right.$ respectively, $\left.d_{1}\left(\bmod s^{1+1} R^{\prime}\right)\right)$ as a power series in positive powers of $y$ (respectively, $x$ ) with coefficients in $s^{l} A^{\prime} / s^{t+1} A^{\prime}$. Moreover, $d_{1} d_{v}=1-u_{\imath}-u_{v}+u_{\imath} u_{v}=d_{1}+d_{v}+\left(u_{\imath} u_{1}-1\right)$. It thus follows that $\left(u_{x} u_{1}-1\right) \in A^{\prime} \cap s^{\prime} R^{\prime}=s^{\prime} A^{\prime}$, hence that $d_{x}+d_{v} \in s^{\prime} A^{\prime}+s^{t+1} R^{\prime}$. But from the representation of $d_{v}\left(\bmod s^{i+1} R^{\prime}\right)$ and $d_{v}\left(\bmod s^{i+1} R^{\prime}\right)$ as power series of the form just described, it thus follows that $d_{r}, d_{v} \in s^{t+1} R^{\prime}$. By induction (and the fact that $R^{\prime}$ is complete and separated in the $s$-adic topology), this completes the proof of the Lemma. $O$

§3.9. We now consider a slightly different definition of an "admissible covering," which is essentially the same as that of [19]. Let $S$ be a scheme. Let $\left(f: C \rightarrow S, \mu_{f} \subseteq C\right)$ and $\left(h: D \rightarrow S, \mu_{h} \subseteq D\right)$ be symmetrically pointed stable curves. Then we make the following definition

Definition. A morphism $\pi: C \rightarrow D$ will be called an HM-admissible covering if it satisfies conditions (2) through (5) of the definition of a log admissible covering, as well as the following condition, consisting of two parts, which we refer to together as "condition $\left(1^{\prime}\right)$ " : $\pi$ is étale, except: 
(1'A) over $\mu_{h}$, where it exhibits tame ramification

( $\left.1^{\prime} \mathrm{B}\right)$ over the singular points: if $s \in S, \lambda$ is a node of $C_{\bar{\zeta}}$, and $v=\pi(\lambda)$, then there exist $a \in m_{S .,}^{s h}, x, y \in m_{C, \lambda}^{s h}$ and $u, v \in m_{D, v}^{s h}$, such that $x y=a$, $u v=a^{e}, u=x^{e}, v=y^{e}$ (for some natural number $e$ (necessarily less than $d$ ) such that $e \in C_{S . s}^{x}$ ) and such that $x, y$ (respectively, $u, v$ )

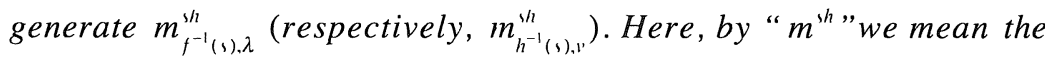
maximal ideal of " $c^{\text {sh }}$," the strict henselization of the local ring in question.

Remark 1. Suppose that condition $\left(1^{\prime} \mathrm{B}\right)$ is satisfied. Then let us assume that we are given elements $u^{\prime}, v^{\prime} \in m_{D, \pi(c)}^{\prime h}$ and $b^{\prime} \in m_{S, s}^{s / h}$ that satisfy $u^{\prime} v^{\prime}=b^{\prime}$ and that generate $m_{h^{-1}(1), d}^{\prime \prime h}$. Then we claim that there exist $x^{\prime}, y^{\prime} \in m_{C, c}^{\prime h}$, together with $a^{\prime} \in m_{S, \text { s. }}^{\text {,h }}$ that satisfy $\left(x^{\prime}\right)^{e}=u^{\prime} ;\left(y^{\prime}\right)^{e}=v^{\prime} ; x^{\prime} y^{\prime}=a^{\prime}$. Indeed, this follows from the fact that the unordered pair $\left(u^{\prime}, v^{\prime}\right)$ differs from the unordered pair $(u, v)$ by multiplication by units (Lemma 7). Taking $e^{\text {th }}$ roots of these units (which is possible precisely because we are working in the strict henselizations), then multiplying by the original $x, y$, and $a$ proves the claim.

Remark 2. Note that unlike in [19], p. 57, in condition (1'B) above, we use strict henselizations rather than completions. The main reason for this is that strict henselizations are often technically easier to work with. Moreover, whether one uses strict henselizations or completions of strict henselizations (i.e., one wants the residue field to be separably closed) in condition $\left(1^{\prime} B\right)$ makes no difference. Indeed, this follows immediately from the argument of the preceding Remark: i.e., whether or not condition $\left(\mathrm{l}^{\prime} \mathrm{B}\right)$ holds is independent of which $u, v$, and $b$ one chooses downstairs; thus, if one knows that a "good" set of $u, v$, and $b$ exist once one passes to the completion of the strict henselization, then it follows that a "good" set already exists in the strict henselization.

$\S 3.10$. We retain the notations of the preceding $\S$. Let $\pi: C \rightarrow D$ be an HMadmissible covering. We would now like to define a canonical $\log$ structure on $S$ (determined completely by $\pi$ ), over which $\pi$ will extend to a log admissible covering $\pi^{\log }$. First let us denote by $M_{S}^{u p}$ (respectively, $M_{S}^{d n}$ ) the monoid which forms the $\log$ structure on $S$ obtained by pulling back that of $\mathscr{M}_{\mathcal{F}_{g, 1}}^{\log }$ (respectively, $\left.\overline{M H S}_{q .1}^{\log }\right)$ via the classifying morphism for $\left(f: C \rightarrow S, \mu_{f}\right)$ (respectively, $\left(h: D \rightarrow S, \mu_{h}\right)$. Let $M_{C}^{u p}$ (respectively, $M_{D}^{d n}$ ) denote the monoid which forms the $\log$ structure on $C$ (respectively, $D$ ) obtained by pulling back that of the universal symmetric stable log-curve. Next let us focus attention at a node $\lambda \in C_{\bar{\zeta}}$. Let $v=\pi(\lambda)$. Let us suppose that we have $x, y, u, v, a, e$ as in Condition ( $\left.1^{\prime} \mathrm{B}\right)$ above. 
Let $b=a^{e}$, so $u v=b$. Let us choose sections (in an étale neighborhood of $\lambda$ ) $\log (x), \log (y)$ of $M_{C}^{u p}$, and a section $\log (a)$ of $M_{S}^{u p}$ such that $\log (x)+\log (y)=$ $\log (a)$, and such that under the exponential map, $\log (x), \log (y)$, and $\log (a)$ map to $x, y$, and $a$, respectively. Note that by Lemma 8 , these properties determine $\log (x), \log (y)$, and $\log (a)$ (as a triple) uniquely. Similarly, we choose sections $\log (u), \log (v)$, and $\log (b)$.

We would like to define a new log structure, whose monoid we denote by $M_{S}$, on $S$. To do this, we note that, étale locally on $S$ near $s$, the $\log$ structure $M_{S}^{u p}$ (respectively, $M_{S}^{d n}$ ) has a chart (in the sense of [21], §2.9) whose generators are indexed by the nodes on $C_{\bar{\zeta}}$ (respectively, $D_{\bar{s}}$ ). More concretely, we may take, for the generator indexed by the node $\lambda$ (respectively, $v$ ), the element $\log (a)$ (respectively, $\log (b)$ ) just defined. We then define $M_{S}$ to be the $\log$ structure associated to the following quotient monoid:

$$
\left(M_{S}^{u p} \times M_{S}^{d n}\right) / \sim
$$

where the equivalence relation " $\sim$ " is that generated by requiring that $(0, \log (b))$ $\sim(e \cdot \log (a), 0)$. By Lemmas 7 and 8 , everything that we are doing is canonical, so even though we defined $M_{S}$ étale locally on $S$, (the log structure defined by) $M_{S}$ descends to our original $S$. This completes the construction of $M_{S}$. Note that we have canonical morphisms $M_{S}^{u p} \rightarrow M_{S}$ and $M_{S}^{d n} \rightarrow M_{S}$.

Now let us denote by $\left(C, M_{C}\right)$ (respectively, $\left(D, M_{D}\right)$ the log scheme obtained as the fiber product $\left(C, M_{C}^{u p}\right) \times_{\left(S, M_{S}^{u \prime \prime}\right)}\left(S, M_{S}\right)$ (respectively, $\left(D, M_{D}^{d n}\right) \times_{\left(S, M_{S}^{d n}\right)}(S$, $\left.M_{S}\right)$ ). We would like to extend the morphism $\pi$ to a morphism of log schemes

$$
\pi^{\log }:\left(C, M_{C}\right) \rightarrow\left(D, M_{D}\right)
$$

over $\left(S, M_{S}\right)$ which is a log admissible covering. We do this as follows. First note that away from the nodes, $\pi$ extends naturally to $\pi^{\text {log }}$ since the only substantive part (i.e., not pulled back from $S$ ) of the log structure is constituted by the divisors of marked points, which are carried to each other (by the definition of an HM-admissible covering). Moreover, away from the nodes, the log morphism $\pi^{\log }$ that we obtain is $\log$ étale, by condition $\left(1^{\prime} \mathrm{A}\right)$ of the definition of an HMadmissible covering. Thus, it remains to deal with the nodes. We can work étale locally, as long as we do things canonically. Thus, we focus attention at a node $\lambda$ of $C_{\bar{\zeta}}$, as above. Then the portion of the $\log$ structure of $M_{D}$ that does not come from $M_{S}$ is generated (in a neighborhood of $v$ ) by $\log (u)$ and $\log (v)$. Thus, it suffices to specify what happens to $\log (u)$ and $\log (v)$ under $\left(\pi^{\log }\right)^{-1}$, and to check that what we specify satisfies the relevant relations. We do this as follows: we send $\log (u)$ to $e \cdot \log (x)$ and $\log (v)$ to $e \cdot \log (y)$. The only relation to be checked is 
$\log (u)+\log (v)=\log (b)$, but this is clearly preserved (by the definition of $\left.M_{S} !\right)$. By Lemmas 7 and 8, everything is canonical, so this morphism $M_{D} \rightarrow M_{C}$ descends to $C$. Moreover, by [21], Theorem 3.5, it is clear that the morphism $M_{D} \rightarrow M_{C}$ thus defined is $\log$ étale. Finally, since $e \leq d$, it follows that the $\log$ morphism $\pi^{\log }:\left(C, M_{C}\right) \rightarrow\left(D, M_{D}\right)$ satisfies condition (1) of the definition of a $\log$ admissible covering.

$\S 3.11$. We summarize the above discussion as follows.

Definition. We shall call the log structure $M_{S}$ on $S$ the canonical log structure associated to the (HM-) admissible covering $\pi$.

Proposition. A morphism $\pi: C \rightarrow D$ between symmetrically pointed stable curves $\left(f: C \rightarrow S, \mu_{f} \subseteq C\right)$ and $\left(h: D \rightarrow S, \mu_{h} \subseteq D\right)$ is an HM-admissible covering if and only if it is an admissible covering in the sense of Definition 6.

Proof. We have just shown the (nontrivial) "only if" part. The "if" part is trivial: it follows immediately by writing out condition (1) of the definition of a $\log$ admissible covering, i.e., what it means to have a log étale morphism.

$\S 3.12$. Before going on to the next subsection, we would like to consider briefly how admissible coverings behave over discrete valuation rings. Let $S=\operatorname{Spec}(A)$, where $A$ is a strict henselian discrete valuation ring.

Lemma. Let $A$ be a strict henselian discrete valuation ring such that $n \in A^{\times}$. Let $s \in A$ be a uniformizer. For each natural number $i \geq 1$, let $R_{t}$ be the strict henselization of $A[X, Y] /\left(X Y-s^{\prime}\right)$ at the maximal ideal $m=(x, y, s)$ (where $x, y$ are the images of $X, Y$ in $R_{n}$ ). (Here $X$ and $Y$ are indeterminates.) Let $W_{l}=\operatorname{Spec}\left(R_{t}\right)-\{m\}$. Then $\pi_{1}\left(W_{n}\right) \cong \mathbb{Z} / n \mathbb{Z}$, and the universal cover $W_{n}^{\sim}$ of $W_{n}$ is $W_{1}$ with the map $W_{n}^{-} \rightarrow W_{n}$ induced by mapping $x$ to $x^{n}$ and $y$ to $y^{n}$.

Proof. The map $W_{1} \rightarrow W_{n}$ is clearly finite étale with $W_{1}$ connected. On the other hand, $R_{1}$ is a regular local ring, so $\pi_{1}\left(W_{1}\right)$ is trivial. The lemma follows. $O$

$\S 3.13$. We continue with the notations of the preceding $\S$. Let $(f: C \rightarrow S, \mu, \subseteq C)$ and $\left(h: D \rightarrow S, \mu_{h} \subseteq D\right.$ ) be symmetrically pointed stable curves over $S$ that are smooth over the generic point $\eta$ of S. Let $\pi_{\eta}: C_{\eta} \rightarrow D_{\eta}$ be an admissible covering over $\eta$. Then

Lemma. There is at most one extension of $\pi_{\eta}$ to an admissible covering $\pi: C \rightarrow D$ over $S$. If $d ! \in A^{\times}$, then (after possibly replacing $A$ by a tamely ramified extension) there exists an admissible covering $\pi: C \rightarrow D$ that extends $\pi_{\eta}$. 
Proof. The uniqueness statement follows immediately from the fact that $C_{\eta}$ is schematically dense in $C$. Now we consider existence: Let $C^{\prime} \rightarrow S$ be the normalization of $D$ in $C_{\eta}$. By Abhyankar's lemma, we may, after possibly replacing $A$ by a tamely ramified extension, assume that $C^{\prime} \rightarrow D$ is not ramified over the generic points of the special fibre of $D$. By purity of the branch locus, the only places where $C^{\prime} \rightarrow D$ might not be étale are over $\mu_{h}$ and over the singular points of $D_{s}$ (where $s$, as usual, is the special point of $S$ ). By Abhyanker's lemma (and the fact that $d ! \in A^{\times}$), we have tame ramification over $\mu_{h}$, so we need only deal with the singular points of $D_{s}$. But at the singular points, it follows from the Lemma of the preceding $\S$ that $C^{\prime} \rightarrow D$ extends to an admissible covering, and $C^{\prime}=C$. $O$

\section{§C。Global Moduli}

$\S 3.14$. Let $S$ be a noetherian scheme. Let $f: X \rightarrow S$ be a flat, proper morphism. Let $\mathscr{L}$ be a relatively ample line bundle on $X$. Then we have the following

Lemma. Let $\phi: \mathscr{F} \rightarrow \mathscr{G}$ be a morphism of coherent sheaves on $X$. Suppose that $\mathscr{G}$ is flat over $S$. Then there exists a unique closed subscheme $Z_{\subsetneq} S$ such that for every morphism $T \rightarrow S$, we have $\phi_{T}=0$ (where the subscript denotes the result of base-changing from $S$ to $T$ ) if and only if the morphism $T \rightarrow S$ factors through $Z$.

Proof. By uniqueness, it suffices to prove the Lemma Zariski locally on $S$. Then there exists an $n$ such that $\mathscr{F}(n)$ (where $\mathscr{F}(n)=\mathscr{F} \otimes,_{x} \mathscr{L}^{\otimes n}$ ) is generated by global sections. Thus, it suffices to prove the Lemma in the case $\mathscr{F}=\mathscr{C}_{X}$. Moreover, if $n$ is sufficiently large, then $f \mathscr{C}_{X}(n)$ and $f \mathscr{G}(n)$ are vector bundles on $S$. Let $\alpha: f \mathscr{C}_{X}(n) \rightarrow f \mathscr{G}(n)$ be the morphism of vector bundles induced by twisting, then pushing forward $\phi$. Let $Z$ be the zero locus of $\alpha$. Then $Z$ has the property asserted in the statement of the Lemma.

Corollary. Let $F$ and $G$ be closed subschemes of $X$, and suppose that $F$ is flat over $S$. Then there exists a closed subscheme $Z \subseteq S$ such that for every morphism $T \rightarrow S$, we have $F_{T} \subseteq G_{T}$ (where the subscripts denote the result of base-changing from $S$ to $T$ ) if and only if the morphism $T \rightarrow S$ factors through $Z$.

Proof. Apply the above Lemma to the morphism $\mathscr{J} \rightarrow C_{F}$, where $G=V(\mathscr{J})$. 
§3.15. Suppose now that $\left(f: C \rightarrow S, \mu_{f} \subseteq C\right)$ and $\left(h: D \rightarrow S, \mu_{h} \subseteq D\right)$ are symmetrically pointed stable curves. Suppose that we are given an $S$-morphism $\pi: C \rightarrow D$.

Lemma. There exists a unique subscheme $Z \subseteq S$ such that for any morphism $T \rightarrow S, \quad \pi_{T}: C_{T} \rightarrow D_{T}$ satisfies conditions (2), (3), and (4) of the definition of a $\log$ admissible morphism if and only if $T \rightarrow S$ factors through $Z$.

Proof. For finiteness (condition (2)), one notes that there exists a closed set $H \subseteq C$ such that $\pi$ restricted to $C-H$ is quasi-finite, while for $x \in H$, the dimension of $\pi^{-1} \pi(x)$ at $x$ is nonzero. Then taking $Z_{2}=S-f(H)$ (which is open in $S$ ) takes care of condition (2). Thus, we may assume that $\pi$ is finite. Note that it then follows (by the local criterion for flatness) that $\pi$ is flat over the smooth locus of $h: D \rightarrow S$. Then let us take a collection of nonintersecting sections $\rho_{1}, \ldots, \rho_{N}$ of $h: D \rightarrow S$ at whose images $h$ is smooth and such that every irreducible component of every fiber of $h$ has a $\rho_{\imath}$ passing through it (note that one can always find such $\rho_{t}$ étale locally on $S$ ). Then $K_{t} \stackrel{\text { der }}{=} C \times_{D, \pi, \rho}, S$ finite and flat over $S$. Moreover, condition (3) is equivalent to the condition that all the $K_{t}$ be of degree $d$ over $S$. Thus, taking the open subscheme $Z_{3} \subseteq S$ over which all the $K$, have degree $d$ takes care of condition (3). Finally, we can take care of Condition (4) by means of Corollary 14 (in the previous $\S$ ). 0

§3.16. Suppose now that $\left(f: C \rightarrow S, \mu_{f} \subseteq C\right)$ and $\left(h: D \rightarrow S, \mu_{h} \subseteq D\right)$ are symmetrically pointed stable curves, and that we are given an $S$-morphism $\pi: C \rightarrow D$ satisfying conditions (2), (3), and (4), of the definition of a log admissible covering. Now let us note that the fact that condition (4) is satisfied means that $\pi$ automatically extends uniquely to a $\log$ morphism $\pi^{\log }$ over the smooth locus of $h: D \rightarrow S$, for any choice of $\log$ structure on $S$. Thus, we can consider the morphism $d \pi: \pi^{\prime} \Omega_{D / S}\left(\mu_{h}\right) \rightarrow \Omega_{C / S}\left(\mu_{f}\right)$ of differentials with logarithmic poles at the marked points. Let us denote by $\omega_{C / S}$ the relative dualizing sheaf of $C$ over $S$. Note that $\omega_{C / S}\left(\mu_{f}\right)$ is naturally isomorphic to $\Omega_{C^{\log / S^{\log }}}$. Indeed, this follows immediately from computing dualizing sheaves in the universal case $\mathscr{C} \rightarrow \overline{\mathbb{M}}_{g, l}$. Thus, we have a natural morphism

$$
\Omega_{C / S}\left(\mu_{f}\right) \rightarrow \Omega_{C^{\log / S^{\log }}}=\omega_{C / S}\left(\mu_{f}\right)
$$

which is an isomorphism outside the singular locus of $f: C \rightarrow S$. In other words, if we denote the zero set of this natural morphism by $C^{\text {sing }}$, then $C^{\text {sing }} \subseteq C$ is a closed subscheme, and $C-C^{\text {smg }} \subseteq C$ is precisely the open set of points at which $f: C \rightarrow S$ is smooth. 
Since the smooth locus of $f: C \rightarrow S$ is schematically dense in $C$, it follows that if there exists a morphism $\psi: \pi \omega_{D / S}\left(\mu_{h}\right) \rightarrow \omega_{C / S}\left(\mu_{f}\right)$ that extends $d \pi$ then it is unique. We shall refer to an isomorphism $\psi: \pi \omega_{D / S}\left(\mu_{h}\right) \rightarrow \omega_{C / S}\left(\mu_{f}\right)$ that extends $d \pi$ as a $\log$ extension of $d \pi$.

Lemma. There exists a unique subscheme $Z \subseteq S$ such that for any $T \rightarrow S$, $d \pi_{T}$ admits a log extension if and only if $T \rightarrow S$ passes through $Z$.

Proof. Let $Z_{1} \subseteq S$ be the subscheme such that for any $T \rightarrow S$, $\left.\left.\left(\pi \omega_{D / S}\left(\mu_{h}\right)\right)\right|_{T} \cong\left(\omega_{C / S}\left(\mu_{f}\right)\right)\right|_{T} \otimes, I^{\prime}$ (for some line bundle ./" on $T$ ) if and only if $T \rightarrow S$ factors through $Z_{1}$. (See [3], Theorem 7.3, for the existence of such a $Z_{1}$.) By restricting to $Z_{1}$, we may assume that $\pi \omega_{D / S}\left(\mu_{h}\right)$ and $\omega_{C / S}\left(\mu_{f}\right)$ are already isomorphic. Let $\rho: S \rightarrow C$ be a section such that $f$ is smooth at $\rho$, and $h$ is smooth at $\pi \circ \rho$. By passing to an open subset of $S$, we may assume that $\rho(d \pi)$ is an isomorphism. Let $\psi$ be that isomorphism of $\pi \omega_{D / S}\left(\mu_{h}\right)$ and $\omega_{C / S}\left(\mu_{f}\right)$ whose restriction to $\rho$ is equal to $\rho(d \pi)$. The fact that there exists a $Z$ as asserted in the statement of the Lemma then follows from applying Lemma 14 to the difference of the two morphisms $\pi \Omega_{D / S}\left(\mu_{h}\right) \rightarrow \omega_{C / S}\left(\mu_{f}\right)$ (one obtained from $d \pi$, the other from $\psi$ ).

Suppose that $d \pi$ admits a log extension. Then it follows immediately from direct calculation of $\omega_{C / S}$ at a smooth point and at a node that condition (5) of the definition of a log admissible morphism is satisfied.

$\S 3.17$. Before we can continue, we need to make certain observations concerning the $\log$ structure of a symmetrically pointed stable log-curve $f^{\log }: C^{\log } \rightarrow S^{\log }$. As before, we denote by $M_{C}$ the étale monoid on $C$ defining the log structure of $C^{\log }$, and by $N_{C}$ the quotient $M_{C} / C_{C}{ }^{x}$. Let $v \in C^{m i n g}$ be a node. Then we make the following

Deffinitiom.We shall call an (unordered) pair of elements $(\alpha, \beta)$ of $\Gamma\left(C, N_{C}\right)$ a separating pair at $v$ if $\alpha \cdot \beta \in \Gamma\left(S, N_{S}\right)$, and, moreover, (étale locally at $v$ ), there exist sections $\tilde{\alpha}, \tilde{\beta}$ of $M_{C}$ such that $\tilde{\alpha}$ (respectively, $\tilde{\beta}$ ) projects to $\alpha$ (respectively, $\beta$ ) in $N_{C}$, and moreover, the images of $\tilde{\alpha}$ and $\tilde{\beta}$ under the morphism $M_{C} \rightarrow C_{C}$ (that forms the log structure) map into and generate the maximal ideal of ${ }^{C} C_{f(1,1,}$.

Note that if $(\alpha, \beta)$ forms a separating pair, then it follows from the definitions that both $\alpha$ and $\beta$ necessarily belong to $\Gamma_{C} \subseteq N_{C}$, at least in a neighborhood of $v$.

It is not necessarily the case that a separating pair at $v$ exists, even étale locally on the base. However, we have the following result: 
Proposition. Suppose that removing $v$ from $C_{f(v)}$ breaks $C_{f(v)}$ up into two separate connected components. Then, étale locally on $S$, there exists a separating pair $\alpha, \beta \in \Gamma\left(C, \Gamma_{C}\right)$ at $v$.

Proof. It suffices to prove this in the universal case. Therefore, we may assume that $S$ is étale over $\bar{M}_{g, l}$. In fact, by basic properties of étale sheaves, we may even assume that $S$ is the completion of $\overline{\mathscr{M}}_{g,}$ at the point defined by $C_{f(v)}$, and that the $\log$ structure on $S$ is that $\log$ structure pulled back from the classifying morphism $S \rightarrow \overline{\mathscr{M}}_{g, l}$. Thus, $M_{C}$ injects into $\mathscr{C}_{C}$. In an étale neighborhood of $v$, there exist elements $x, y$ of $m_{C, 1}$ (i.e., the maximal ideal of $\left.c_{C, v}\right)$ and $t$ of $m_{S, f(v)}$ such that $x y=t$, and such that $x, y$ generate $m_{C_{t(1,1}}$. We then define $\alpha$ as follows: In a neighborhood of $v$ where $x$ is defined and there are no nodes other than $v$, we let $\alpha$ be the element of $N_{C}$ defined by $x \in M_{C} \subseteq \epsilon_{C}$. Away from $v$, we define $\alpha$ as follows: Over the connected component where $x$ is not identically zero, we take $\alpha$ to be the trivial section of $N_{C}$. Over the connected component where $x$ is identically zero, we take $\alpha$ to be the section of $N_{C}$ defined by $t \in M_{C} \subseteq \mathcal{C}_{C}$. It is easy to see that $\alpha$, defined in this way is welldefined and forms a section of $\Gamma_{C}$. We define $\beta$ by reversing the roles of $x$ and $y$. It is clear that $(\alpha, \beta)$ then forms a separating pair at $v$. 0

$\S 3.18$. We continue with the notation of the preceding $\S$. So what happens when the node $v$ does not satisfy the hypotheses of the preceding Proposition? This happens when the dual graph of $C_{f(p)}$ has a loop. In this case, however, it is easy to see that there exists a finite étale covering of degree two: $\phi_{f(1)}: \tilde{C}_{f(1)} \rightarrow C_{f(1)}$ such that

(1) $\phi^{-1}(v)$ consists of two points $v_{1}$ and $v_{2}$

(2) over $C_{f(1)}-v, \tilde{C}_{f(v)}$ consists of two disjoint copies of $C_{f(1)}-v$.

Moreover, étale locally on $S$, there exists a finite étale covering of degree two $\phi: \tilde{C} \rightarrow C$ whose restriction to $f(v)$ is $\phi_{f\left(v^{\prime}\right)}$. Let us equip $\tilde{C}$ with the log structure pulled back from $C$, and denote the resulting log scheme by $\tilde{C}^{\text {log }}$. Moreover, we let $\Gamma_{\tilde{C}}=\phi \Gamma_{C}$. Then we have the following result:

Proposition. Étale locally on $S$, there exist elements $\alpha, \beta \in \Gamma\left(\tilde{C}, \Gamma_{\tilde{C}}\right)$ that form a separating pair at both $v_{1}$ and $v_{2}$.

Proof. The proof is completely analogous to the proof of the Proposition in the preceding $\S$. 
§3.19. Suppose now that $\left(f: C \rightarrow S, \mu_{f} \subseteq C\right)$ and $\left(h: D \rightarrow S, \mu_{h} \subseteq D\right)$ are symmetrically pointed stable curves, and that we are given an $S$-morphism $\pi: C \rightarrow D$ satisfying conditions (2), (3), (4), and (5) of the definition of a log admissible covering. Let $M_{S}^{u p}$ and $M_{S}^{d n}$ be as in $\S 3.10$. Let $N_{S}^{u p}=M_{S}^{u p} / \mathscr{O}_{S}^{\times}$; and $N_{S}^{d n}=M_{S}^{d n} / \mathscr{O}_{S}{ }^{\times}$. Also, we have subsheaves of generators $\Gamma_{S}^{u p} \subseteq N_{S}^{u p}$, and $\Gamma_{S}^{d n} \subseteq N_{S}^{d n}$, as defined in $\S 3.3$. Since $\Gamma_{S}^{u p}$ and $\Gamma_{S}^{d n}$ are constructible sheaves of sets on $S$, it follows that they can be represented ([28], p. 160) by quasi-finite étale algebraic spaces over $S$; in the following we shall identify these sheaves with the spaces that represent them. Let $\Delta_{S}^{u p}$ be the subsheaf of $N_{S}^{u p}$ obtained by considering sums of $d$ sections of $\Gamma_{S}^{u p}$. Let $Z_{1} \rightarrow S$ be the quasi-finite étale algebraic space over $S$ parametrizing morphisms

$$
\Gamma_{S}^{d n} \rightarrow \Delta_{S}^{u p}
$$

that preserve the zero section. Note that $Z_{1}$ need not be a separated $S$-scheme.

Let us focus attention on a geometric point $\bar{s}$ of $S$. Let us assume that we are given a collection $\gamma_{1}, \ldots, \gamma_{N} \in \Gamma\left(S, \Gamma_{S}^{d n}\right)$ such that the morphism of sheaves of sets $\{1, \ldots, N\} \rightarrow \Gamma_{S}^{d n}$ given by mapping $i$ to $\gamma_{1}$ is surjective, and $N$ is equal to the number of nodes of $D_{\bar{s}}$. (One can always find such a collection of $\gamma_{1}$ 's by étale localization at $\bar{s}$.) Thus, over $Z_{1}$, we have a tautological morphism $\sum:\left.\Gamma_{s}^{d n}\right|_{Z_{1}}$ $\left.\rightarrow \Delta_{S}^{u p}\right|_{Z_{1}}$, together with sections $\gamma_{1} \in \Gamma\left(Z_{1}, \Gamma_{S}^{d n}\right) \subseteq \Gamma\left(Z_{1}, N_{S}^{d n}\right)$, and sections $\gamma_{1}^{\prime}=$ $\sum\left(\gamma_{1}\right) \in \Gamma\left(Z_{1}, N_{S}^{u p}\right)$. Let $\mathscr{L}_{\gamma_{1}}$ (respectively, $\mathscr{L}_{\gamma_{1}^{\prime}}$ ) be the line bundle on $Z_{1}$ corresponding to the $C_{Z_{1}}{ }^{x}$-torsor obtained by considering liftings of $\gamma$ (respectively, $\gamma^{\prime}$ ) to a section of $M_{S}^{d n}$ (respectively, $M_{S}^{u p}$ ). Let $Z_{2} \rightarrow Z_{1}$ be the space of $N$-tuples of isomorphisms $\mathscr{L}_{\gamma_{1}} \cong \mathscr{Z}_{\gamma_{1}^{\prime}}$ over $Z_{1}$. Thus, $Z_{2}$ is a $\mathbb{G}_{m}$-torsor over $Z_{1}$. Note that if we know that $\pi: C \rightarrow D$ is admissible, then we obtained a natural section of $Z_{2}$ in the course of constructing the canonical log structure on $S$. More concretely, in the notation of $\$ 3.10$, the morphisms $\Gamma_{S}^{d n} \rightarrow \Delta_{S}^{u p}$ and $\mathscr{L}_{\gamma_{1}} \rightarrow \mathscr{L}_{\gamma^{\prime}}^{\prime}$ are those induced by the morphism $M_{S}^{d n} \rightarrow M_{S}^{u p}$ given by $\log (b) \mapsto \sum e \cdot \log (a)$, where the sum is over all nodes $\lambda$ of $C_{\bar{\zeta}}$ that map to the node in question $v$ (i.e., the node for which $b$ is a deformation parameter) of $D_{\bar{s}}$.

Let $Z_{3} \subseteq Z_{2}$ be the closed sub-algebraic space over which the tautological isomorphisms $\mathscr{L}_{\gamma_{1}} \rightarrow \mathscr{L}_{\gamma^{\prime}}$ are compatible with the exponential morphisms $\mathscr{L}_{\gamma_{1}} \rightarrow C_{Z_{2}}$ and $\mathscr{L}_{\gamma_{1}^{\prime}} \rightarrow C_{Z_{2}}$ of the log structures $M_{S}^{d n}$ and $M_{S}^{u p}$. Let $M^{\text {ad }} \rightarrow C_{Z_{2}}$ be the $\log$ structure on $Z_{3}$ associated to the largest quotient monoid $Q$ of $\left.\left(M_{S}^{u p} \times M_{S}^{d n}\right)\right|_{Z_{3}}$ such that the following diagram commutes: 


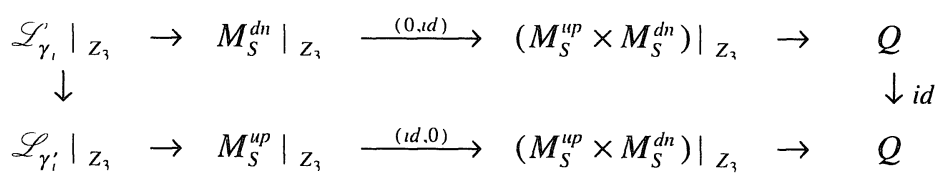

Thus, we obtain a log algebraic space $\left(Z_{3}, M^{a d}\right)$, together with logarithmic morphisms $\left(Z_{3}, M^{a d}\right) \rightarrow\left(Z_{3},\left.M_{S}^{u p}\right|_{Z_{3}}\right) \rightarrow \overline{\mathscr{N} S}_{g, r}^{\log }$, and $\left(Z_{3}, M^{\text {ad }}\right) \rightarrow\left(Z_{3},\left.M_{S}^{\text {dn }}\right|_{Z_{3}}\right) \rightarrow$ $\overline{\mathscr{N S}}_{q, s}^{\log }$, which thus determine log-curves $C_{Z_{3}}^{\log } \rightarrow\left(Z_{3}, M^{a d}\right)$ and $D_{Z_{3}}^{\log } \rightarrow\left(Z_{3}, M^{a d}\right)$ (whose underlying curves are $C \rightarrow S$ and $D \rightarrow S$, pulled back to $Z_{3}$ ). Let us denote the monoids defining the $\log$ structures on $C_{Z_{3}}^{\log }$ and $D_{Z_{3}}^{\log }$ by $M_{C}$ and $M_{D}$, respectively. Thus, as usual, we obtain sheaves $N_{C}, \Gamma_{C}, N_{D}$, and $\Gamma_{D}$.

$\S 3.20$. We maintain the notation of the preceding $\S$. Let us focus attention on a node $v$ of $D_{\bar{s}}$. Let us assume that we have a separating pair $(\alpha, \beta)$ at $v$ (for the $\log$-curve $D_{Z_{3}}^{\log } \rightarrow\left(Z_{3}, M^{\text {ad }}\right)$ ). Also, let us assume that $\gamma \stackrel{\text { def }}{=} \alpha \cdot \beta$ is equal to the image of $\gamma_{1}$ in $M^{a d}$. Let $Z_{4}^{v} \rightarrow Z_{3}$ be the quasi-finite étale algebraic space of pairs of sections $\tilde{\alpha}, \tilde{\beta}$ of $\left.\Gamma_{C}^{d}\right|_{z_{3}}$ such that $\tilde{\alpha} \cdot \tilde{\beta}=\alpha \cdot \beta$. (Here we use the fact that the push-forward of a constructible sheaf by a proper morphism is again constructible - see, e.g., [28], p. 223, Theorem 2.1.) Let $\mathscr{L}_{\alpha}$ and $\mathscr{L}_{\beta}$ be the $\mathscr{O}_{D_{z_{3}}}^{\times}$torsors of liftings to sections of $G_{D}$ of $\alpha$ and $\beta$, respectively. Similarly, we have $\mathscr{L}_{\tilde{\alpha}}$ and $\mathscr{L}_{\tilde{\beta}}$ on $\mathscr{C}_{C_{Z_{z}}}$. Let $Z_{5}^{v} \rightarrow Z_{4}^{v}$ be the relative scheme parametrizing pairs of isomorphisms $\phi: \pi^{\prime} \mathscr{L}_{\alpha} \cong \mathscr{L}_{\tilde{\alpha}}$ and $\psi: \pi^{\prime} \mathscr{L}_{\beta} \cong \mathscr{L}_{\tilde{\beta}}$ that are compatible with the exponential morphisms of the $\log$ structures of $C^{\log }$ and $D^{\log }$, and such that the composite morphism

$$
f^{\prime} \mathscr{L}_{\gamma}^{\prime} \cong \pi^{\prime}\left(\mathscr{L}_{\alpha}^{\prime} \otimes \mathscr{L}_{\beta}\right) \stackrel{\phi \otimes \psi}{\longrightarrow} \mathscr{L}_{\tilde{\alpha}} \otimes \mathscr{L}_{\bar{\beta}}^{\prime} \cong f^{\prime} \mathscr{L}_{\gamma}
$$

(where the two isomorphisms at either end are those induced by the equalities $\alpha \cdot \beta=\gamma=\tilde{\alpha} \cdot \tilde{\beta})$ is the identity morphism. Note that if we know that $\pi: C \rightarrow D$ is admissible, then we automatically obtain canonical $\tilde{\alpha}, \tilde{\beta}, \phi$, and $\psi$ by considering the morphism of log structures (that we get automatically from Proposition 11).

Of course, in general, a separating pair $(\alpha, \beta)$ might not exist. But just as in Proposition 18, we can always find such a pair by passing to an appropriate double covering of $D$. Then it is easy to see that we can still define a $Z_{5}^{r}$ as above (by working with the double coverings of $C$ and $D$ ). Thus, we obtain $Z_{5}^{\prime \prime}$ 's for every 
node $v$ on $D_{\bar{s}}$. Let $Z_{6} \rightarrow Z_{3}$ be the product over $Z_{3}$ of the various $Z_{5}^{v}$, as $v$ varies over the nodes of $D_{\bar{s}}$. Then we have the following

Lemma. The morphism $\left.\pi\right|_{Z_{6}}:\left.\left.C\right|_{Z_{6}} \rightarrow D\right|_{Z_{6}}$ is an admissible covering.

Proof. Indeed, it suffices to check condition $\left(1^{\prime} \mathrm{B}\right)$ of the definition of an HM-admissible covering ( $\$ 3.9)$. But this follows immediately from considering local generators (near a node) of the line bundles $\mathscr{L}_{\alpha}, \mathscr{L}_{\beta}, \mathscr{L}_{\bar{\alpha}}$, and $\mathscr{L}_{\tilde{\beta}}$ above, and writing out what it means to give morphisms $\phi$ and $\psi$ (as in the definition of $Z_{5}^{r}$ ) that are compatible with the exponential morphisms of the log structures, and whose tensor product $\phi \otimes \psi$ satisfies the condition specified in the definition of $Z_{5}^{\prime}$. $\mathrm{O}$

§3.21. We maintain the notations of the preceding $\S$. Now that we know that $\left.\pi\right|_{z_{6}}$ is admissible, we can consider the canonical morphisms $\mathbb{\Gamma}_{S}^{d n} \rightarrow \Delta_{S}^{u p}$; $\mathscr{L}_{\gamma_{1}} \rightarrow \mathscr{L}_{\gamma_{i}} ; \mathscr{L}_{\alpha} \rightarrow \mathscr{L}_{\tilde{\alpha}} ; \mathscr{L}_{\beta} \rightarrow \mathscr{L}_{\bar{\beta}}$ arising from the logarithmic morphisms obtained over the canonical log structures of $\S 3.10,3.11$. Let $Z_{7} \rightarrow Z_{6}$ be the open subalgebraic space on which the canonical $\Gamma_{S}^{d n} \rightarrow \Delta_{S}^{u p}$ agrees with the tautological $\Gamma_{S}^{d n} \rightarrow \Delta_{S}^{u p}$ given by the definition of $Z_{1}$. Let $Z_{8} \subseteq Z_{7}$ be the closed sub-algebraic space on which the canonical $\mathscr{L}_{\gamma_{1}} \rightarrow \mathscr{L}_{\gamma_{i}^{\prime}}$ agrees with the tautological $\mathscr{L}_{\gamma_{1}} \rightarrow \mathscr{L}_{\gamma^{\prime}}$ given by the definition of $Z_{2}$. Similarly, we let $Z_{9} \subseteq Z_{8}$ be the sub-algebraic space on which the canonical $\tilde{\alpha} ; \tilde{\beta} ; \mathscr{L}_{\alpha} \rightarrow \mathscr{L}_{\tilde{\alpha}} ; \mathscr{L}_{\beta} \rightarrow \mathscr{L}_{\tilde{\beta}}$ agree with the tautological $\tilde{\alpha} ; \tilde{\beta} ; \mathscr{Z}_{\alpha} \rightarrow \mathscr{L}_{\tilde{\alpha}} ; \mathscr{Z}_{\beta} \rightarrow \mathcal{Z}_{\tilde{\beta}}$ given by the definitions of $Z_{4}^{v}$ and $Z_{5}^{v}$. Thus, in summary, we have proven the following:

Lemma. Let $T \rightarrow S$ be a morphism of schemes. Then $\pi_{T}: C_{T} \rightarrow D_{T}$ is admissible if and only if $T \rightarrow S$ factors through $Z_{9}$. Moreover, this factorization of $T \rightarrow S$ through $Z_{9}$ is unique. In particular, the algebraic space $Z_{9}$ is, in fact, separated over $S$. Finally, if $d$ ! is invertible on $S$, then $Z_{9}$ is even a closed subscheme of $S$.

Proof. Indeed, it suffices to prove the final statement. This follows by noting that Lemma 13 implies that the morphism $Z_{9} \rightarrow S$ is proper. Since it is also a monomorphism, it follows that it is quasi-finite, hence (by Zariski's main theorem) finite. Thus, $Z_{9}$ is a closed subscheme of $S$. $O$

Note finally, that $Z_{9}$ comes equipped with a canonical log structure, i.e., the $\log$ structure associated to the admissible covering $\left.\pi\right|_{z_{9}}$. 
Remark. In fact, it is not difficult to show (although we will not use this fact) that the morphism $Z_{9} \rightarrow S$ is open (i.e., the image of an open set is open). However, even when $d$ ! is invertible on $S$, it is not necessarily the case either that $Z_{9}$ and $S$ coincide as sets, or that $Z_{9} \hookrightarrow S$ is an open immersion. Indeed, below (in $\S 3.24$ ), we shall construct examples (where $d$ ! is invertible on $S$ ) of points of $S$ which are not in $Z_{9}$, as well as of cases where the nilpotent ideal defining the closed subscheme $Z_{9} \subseteq S$ is nonzero.

$\S 3.22$. We are now ready to state the main Theorem of this Section:

Theorem. Fix nonnegative integers $g, r, q, s, d$ such that $2 g-2+r=$ $d(2 q-2+s) \geq 1$. Let $\propto$ be the stack over $\mathbb{Z}$ defined as follows: for a scheme $S$, the objects of $\mathscr{\Omega}(S)$ are admissible coverings $\pi: C \rightarrow D$ of degree $d$ from a symmetrically r-pointed stable curve $\left(f: C \rightarrow S ; \mu_{f} \subseteq C\right)$ of genus $g$ to a symmetrically s-pointed stable curve $\left(h: D \rightarrow S ; \mu_{h} \subseteq D\right)$ of genus $q$; and the morphisms of $\mathscr{\Omega}(S)$ are pairs of $S$-isomorphisms $\alpha: C \rightarrow C$ and $\beta: D \rightarrow D$ that stabilize the divisors of marked points such that $\pi \circ \alpha=\beta \circ \pi$. Then $\mathscr{A}$ is $a$ separated algebraic stack of finite type over $\mathbb{Z}$. Moreover, $\circlearrowleft$ is equipped with a canonical $\log$ structure $M, \rightarrow{ }^{\top}$, , together with a logarithmic morphism

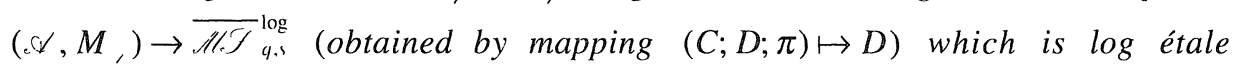
(always) and proper over $\mathbb{Z}\left[\frac{1}{d !}\right]$.

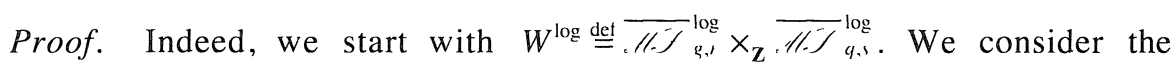
Hilbert stack $H \rightarrow W$ (i.e., $H \rightarrow W$ is a relative scheme) of morphisms from the tautological $r$-pointed curve of genus $g$ to the tautological $s$-pointed curve of genus $q$. Applying Lemmas 15 and 16, we obtain a sub-algebraic stack $S \subseteq H$, to which we can apply the constructions of $\$ 3.19$. Finally, we have the stack $Z_{9} \rightarrow S$ (which was constructed étale locally on $S$, but which clearly descends back down to the original $S$ by Lemma 21). This gives us our $r$. . The fact that the morphism $\left(. \mathcal{S}, M_{3}\right) \rightarrow \overline{\pi / .}_{4.3}^{\log }$ is $\log$ étale is an exercise in tracing through the definitions of the various log structures involved. The assertion on properness then follows from Lemma 13 . $\mathrm{O}$

Remark. Applying the construction of [11], Chapter $1, \S 4.10$, to .7 , one obtains a coarse moduli space $v^{c}$. Moreover, over $\mathbb{Z}\left[\frac{1}{d !}\right], \quad \vee^{c}$ is a projective scheme: Indeed, let $\|^{\prime}$ be the coarse moduli space associated to $\overline{\mathbb{H}}_{4} \ldots$ It is well-known (at least in characteristic zero - see [23]) that ./" is a normal 
projective scheme. Then by the above Theorem, $\mathscr{A}^{c} \rightarrow \mathscr{M}^{c}$ is proper. Also, it is clear (by considering the number of possibilities for an admissible morphism between two given stable curves) that $\mathscr{A}^{c} \rightarrow \mathscr{M}^{c}$ is quasi-finite. Thus, by Zariski's main theorem, it follows that $\mathscr{A}^{c} \rightarrow \mathscr{M}^{c}$ is finite. Thus, $\mathscr{A}^{c}$ is a projective scheme.

\$3.23. Finally, just as in [19], p. 62, we can make explicit the completion of a local ring of $\mathscr{A}$. Let $k$ be an algebraically closed field. Let $A=W(k)$, the ring of Witt vectors with coefficients in $k$. Let $\pi: C \rightarrow D$ be an admissible covering over $k$. Let us denote by $[\pi] \in \mathscr{A}(k) ; \quad[C] \in \overline{\mathscr{M S}}_{g, r}(k) ; \quad[D] \in \overline{\mathscr{M S}}_{q, s}(k)$ the corresponding $k$-valued points of the respective moduli stacks. Let $\mathscr{O}_{[\pi]}$ (respectively, $\mathscr{O}_{[D]}$ ) be the completion of the strict henselization of $\mathscr{A}$ (respectively, $\widetilde{\mathscr{N} S}_{q, s}$ ) at $[\pi]$ (respectively, $[D]$ ). Suppose that $D$ has exactly $N$ nodes, which we number from 1 to $N$. Thus, we have a noncanonical isomorphism.

$$
\mathscr{Q}_{[D]} \cong A\left[\left[t_{1}, \ldots, t_{N}, s_{1}, \ldots, s_{3 q-3+s-N}\right]\right]
$$

where the $t$ 's and $s$ 's are indeterminates; and $t_{i}$ defines a universal deformation parameter for the $i^{\text {th }}$ node on $D$. Suppose that $C$ has $n_{t}$ nodes over the $i^{\text {th }}$ node on $D$, which we label $(i, 1)$ through $\left(i, n_{l}\right)$. Suppose that the ramification index of (either branch of) $C$ over $D$ at the node $(i, j)$ is $e_{t, j}$. Then

$$
C_{[\pi]} \cong C_{[D]}\left[\left\{t_{t, J}\right\}\right] /\left(t_{t, J}^{e_{t,}}-t_{t}\right)
$$

where we allow $i$ to run from 1 to $N$, and $j$ to run from 1 to $n_{l}$. The canonical log structure at $[\pi]$ is the log structure associated to the quotient monoid of the free monoid on the symbols $\left\{\log \left(t_{t, j}\right) ; \log \left(t_{l}\right)\right\}$ (where $i$ and $j$ vary as before) by the equivalence relation generated by $\log \left(t_{t}\right)=e_{l, j} \cdot \log \left(t_{t, j}\right)$. The exponential morphism of the canonical $\log$ structure maps the symbol $\log \left(t_{i, J}\right)$ to (the image in $\mathscr{Q}_{[\pi]}$ of) $t_{t, j}$ and the symbol $\log \left(t_{i}\right)$ to (the image in $\mathscr{O}_{[\pi]}$ of) $t_{i}$.

This sort of explicit structure result gives us the following:

Corollary. Let $\mathscr{A}^{\prime} \subseteq \mathscr{A}$ be the open substack where the curves $C$ and $D$ of the tautological admissible covering $\pi: C \rightarrow D$ are smooth. Then $\mathscr{A}^{\text {' }}$ is smooth over $\mathbb{Z}$ and schematically dense in $\mathscr{A}$. Moreover, $\mathscr{A}$ is flat over $\overline{\mathscr{M}}_{q, s}$; reduced; and its normalization $\mathcal{A}$ ' is smooth over $\mathbb{Z}$ and flat over $\overline{\mathscr{M S}}_{q . s}$. Thus, we have a natural inclusion $\mathscr{A}^{\mathrm{S}} \hookrightarrow \mathscr{N}^{\mathrm{S}}$. Finally, the complement of $\mathscr{A}^{\mathrm{s}}$ in $\mathscr{N}^{\mathrm{s}}$ is a 
divisor with normal crossings. We shall refer to these divisors as the divisors at infinity of $\mathscr{N}$.

$\S 3.24$. We maintain the notations of the preceding $\S$. In this $\S$, we would like to construct examples showing the extent to which (in the notation of Lemma 21) $Z_{9} \neq S$ :

Example 1. First, we show how to construct $k$-valued points of $Z_{9}$ that are not in $S$. Namely, we consider admissible $\pi: C \rightarrow D$ such that $D$ has a node $v$ over which two distinct indices of ramification $e$ and $e^{\prime}$ occur (i.e., $e \neq e^{\prime}$ ). Let $\lambda$ (respectively, $\lambda^{\prime}$ ) be the point of $C$ where the index of ramification $e$ (respectively, $e^{\prime}$ ) occurs. Let us assume (for simplicity) that $v$ sits on two distinct irreducible components $I_{D}$ and $J_{D}$ of $D$. Let $I_{C}$ (respectively, $J_{C}$ ) be the irreducible component of $C$ containing $\lambda$ that lies over $I_{D}$ (respectively, $J_{D}$ ). Similarly, we define $I_{C}^{\prime}$ and $J_{C}^{\prime}$ for $\lambda^{\prime}$. Let $\tilde{C}$ be the curve obtained from $C$ as follows: $\tilde{C}$ is exactly the same as $C$ except at $\lambda$ and $\lambda^{\prime}$; there we glue $I_{C}$ (at $\lambda$ ) to $J_{C}^{\prime}\left(\right.$ at $\left.\lambda^{\prime}\right)$ and $I_{C}^{\prime}\left(\right.$ at $\left.\lambda^{\prime}\right)$ to $J_{C}$ (at $\lambda$ ). Then we have a map $\tilde{\pi}: \tilde{C} \rightarrow D$ (induced by $\pi$ ) which satisfies conditions (2) through (5) of the definition of a $\log$ admissible covering, but is obviously not admissible. (In fact, if $e \equiv e^{\prime}(\bmod p)$ (where $p=\operatorname{char}(k)$ ), then $d \pi^{\prime}$ even admits a log extension in the sense of $\$ 3.16$. Of course, $e \equiv e^{\prime}(\bmod p)$ is impossible if $d ! \in k^{\times}$.)

Example 2. Let $B=k[\varepsilon] /\left(\varepsilon^{2}\right)$. Let $S=\operatorname{Spec}(B)$; let $s \in S(k)$ be the unique point. Let $D_{\varepsilon}=D \times_{k} S ;\left(\mu_{h}\right)_{\varepsilon}=\left(\mu_{h}\right) \times_{k} S \subseteq D_{\varepsilon}$, where $\mu_{h} \subseteq D$ is the divisor of marked points in $D$. We would like to consider morphisms $\pi_{\varepsilon}: C_{\varepsilon} \rightarrow D_{\varepsilon}$ from a symmetrically pointed stable curve $\left(f_{\varepsilon}: C_{\varepsilon} ;\left(\mu_{f}\right)_{\varepsilon} \subseteq C_{\varepsilon}\right)$ to $\left(D_{\varepsilon} ;\left(\mu_{h}\right)_{\varepsilon}\right)$ such that $\left.\pi_{\varepsilon}\right|_{1}=\pi$; and $\pi_{\varepsilon}$ satisfies conditions (2) through (5) of the definition of a $\log$ admissible morphism. By deformation theory, it follows that the isomorphism classes of such $\pi_{\varepsilon}$ are in one-to-one correspondence with the elements of some finite-dimensional $k$-vector space $V$, which can be computed by means of local computations at the nodes. In fact, it is not difficult to calculate $V$ explicitly. One thus obtains that

$$
\operatorname{dim}_{h} V=\sum_{t, J} 2\left(e_{t, J}-1\right)+\operatorname{sgn}\left(e_{t, J}-1\right)
$$

where $i, j$ run over all $i$ and $j$ as allowed in $\S 3.23$, and $\operatorname{sgn}(0)=0$; $\operatorname{sgn}$ of a positive number is 1 . On the other hand, the subspace of $V$ corresponding to admissible coverings $\pi_{\varepsilon}$ is (by what we did in \$3.23) of dimension $\sum_{l, J} \operatorname{sgn}\left(e_{t, J}-1\right)$, i.e., in general, it is much smaller than all of $V$. Thus, we see 
that even at a point of $S$ contained in $Z_{9}$, the ideal defining the closed subscheme $Z_{9}$ is, in general, nonzero.

\section{§31). Admissible Hurwitz Coveriongs}

$\S 3.25$. Let $\pi: C \rightarrow D$ be an admissible covering.

Definitiom.We shall call $\pi$ an admissible Hurwitz covering if the genus of $D$ is zero and $\pi$ is simply ramified over the marked points of $D$.

In this Section, we have been concerned with proving the very general Theorem 22 above. In the rest of this paper, however, we will be concerned only with Hurwitz coverings (i.e., the sorts of coverings parametrized by the Hurwitz stacks of §1) and their degenerations, so we shall refer to admissible Hurwitz coverings simply as admissible coverings.

$\S 3.26$. Remark. In this $\S$, we explain why the proof of Theorem 4, p. 58 of [19] has a fundamental gap. On p. 60 of [19], it is claimed that (in the notation of [19]) $H_{4}$ will represent the functor $\overline{\mathscr{H}}_{h, b}^{U}$. In the notation of our Lemma 21, this essentially amounts to the assertion that $Z_{9}=S$. Indeed, the only difference between our $S$ and [19]'s $H_{4}$ is that $H_{4}$ includes projective embedding data for $C$ (as described on p. 59 of [19]). On the other hand, we saw (Examples 1 and 2 of $\S 3.24)$ that in general, $Z_{9} \neq S$.

To put the issue another way, [19] completely neglect the nontrivial issue of showing that condition $\left(1^{\prime} \mathrm{B}\right)$ of the definition of an $\mathrm{HM}$-admissible covering is "scheme-like," i.e., that given an $S$-morphism $\pi: C \rightarrow D$ satisfying all the conditions for an admissible covering except $\left(1^{\prime} \mathrm{B}\right)$, the set of $s \in S$ over which $\pi_{s}: C_{s} \rightarrow D$, also satisfies $\left(1^{\prime} \mathrm{B}\right)$ has the natural structure of a subscheme $Z$ of $S$, and, moreover, that over $Z, \pi_{Z}: C_{Z} \rightarrow D_{Z}$ is an admissible covering. These issues occupy the bulk of our proof of Theorem 22, and are the main reason for the introduction of log structures. Indeed, the condition that a function defined only locally in a neighborhood of a node on $C$ have an $e^{\text {th }}$ root (say, étale locally) is in general not a very "scheme-like" condition.

For instance, let $S=\operatorname{Spec}(k[[t]]) ; X=\operatorname{Spec}(k[[x, t]])$, where $k$ is an algebraically closed field of characteristic $\neq 2$. We regard $X$ as an $S$-scheme. Consider the condition that the function $x^{2}-t^{2}$ have a square root étale locally in a neighborhood of the point $x=0$. This condition is satisfied at both the special and the generic points of $S$. But it is certainly not satisfied over $S$. 
Thus, in order to obtain a nice moduli problem it is necessary to translate this sort of local condition (i.e., condition $\left(1^{\prime} \mathrm{B}\right)$ ) into a condition which is global on the curve $C$. This is precisely what is achieved by the introduction of $\log$ structures.

$\S 3.27$. Notation. We close by introducing some notation that will be of use in the rest of this paper. Let $b \geq 4$ be an integer. Let $R=\mathbb{Z}\left[\frac{1}{b !}\right]$. Relative to the notation of the present section, we are interested in the case $q=0, s=b$. We denote by $\overline{\mathscr{R} C}_{b}$ (respectively, $\overline{\mathscr{R} P}_{b}$ ) the scheme $\overline{\mathscr{M}}_{q, s} \otimes_{\mathrm{z}} R$ (respectively, stack $\overline{\mathscr{A S}}_{q, s} \otimes_{\mathrm{z}} R$ ), which thus forms a compactification of the scheme $\mathscr{R} C_{b}$ (respectively, stack $\mathscr{R S}_{b}$ ) of $\S 1.3$. By simple combinatorial analysis, one sees that in the genus zero case, in fact, every irreducible component of a stable curve $D$ of genus zero over an algebraically closed field is smooth and rational and that the graph obtained by taking the irreducible components of $D$ as vertices and the singular points (which necessarily join two distinct components) as edges must have $H^{1}=0$ (in the sense of singular homology). We shall call this graph the "graph associated to $D . "$

It follows from [22] that the complement of $\mathscr{R}_{b}$ (resp. $\mathscr{H F}_{b}$ ) in $\overline{\mathscr{R} C_{b}}$ (resp. $\overline{\mathscr{R} S}_{b}$ ), i.e. "the divisor at infinity" of $\overline{\mathscr{H} C}_{b}$ (resp. $\overline{\mathscr{K} S}_{b}$ ), is made up of irreducible components $I$, where the generic point of $I$ represents a stable curve that consists of two copies of $\mathbb{P}^{1}$ joined at one point with $i$ of the marking points on one component and $b-i$ points on the other component. In the symmetrized case, the divisors at infinity are thus in one-to-one correspondence with unordered pairs of natural numbers $(i, j)$ such that $i+j=b, i \geq 2, j \geq 2$. Thus, in the future, we shall speak of the " $(i, j)$-component" or " $(i, j)$-divisor" (at infinity) and so on.

Let $d \geq 2$ be an integer. Since we have already fixed $q=0$ and $s=b$, Theorem 22 gives us an algebraic stack $\mathscr{V}$. Let $\delta \mathscr{V}_{R}=\mathscr{V} \otimes_{\mathrm{Z}} R$. Note that by Abhyankar's Lemma, there is an open and closed substack $\mathscr{\&} \mathcal{J}_{b, d}$ of $\mathscr{\searrow}_{R}$ parametrizing the admissible Hurwitz coverings. Thus, $\mathcal{E J}_{b, d}$ contains $\mathscr{Y M Y S}_{b, d}$ as a dense open substack (Corollary 23). By $\$ 2.9$, it thus follows that $\varepsilon_{b . d}$ is geometrically irreducible over $R$. Let $\& \mathbb{C}_{b, d} \rightarrow \mathscr{E} S_{b . d}$ be the finite étale covering of degree $b$ ! obtained by ordering the marked points of the curve of genus 0 downstairs (i.e., the " $D$ " of $\pi: C \rightarrow D$ ). Let,$I_{b, d}$ (respectively,,$I_{b . d}$ ) be the normalization of $\mathscr{E} \mathcal{S}_{b, d}$ (respectively, $\left.\mathscr{G} /_{b, d}\right)$. Finally, note that we have a commutative diagram 


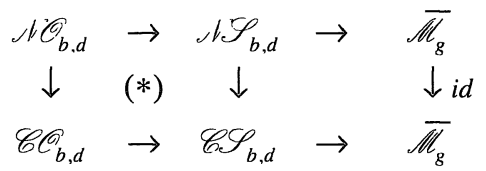

where the horizontal morphisms on the right assign to an admissible covering $\pi: C \rightarrow D$ the curve $C$, and the square $\left(^{*}\right)$ is Cartesian.

\$3.28. Remark. The compactification of the Hurwitz scheme has many interesting applications to the geometry of $\mathscr{M}_{g}$. For instance, as in [27] (the Appendix to [19]), one sees that it allows a direct algebraic proof of the connectedness of $\mathscr{M}_{\mathrm{g}}$. One may also note that the proof of the properness of the compactification $\mathscr{C} \mathscr{C}_{b, d}$ never uses the Semi-Stable Reduction Theorem. Thus it follows that (at least for high characteristic) we obtain a new, direct, elementary proof of this Theorem.

\section{§4. Construction of the Boundary Componemts}

\$4.1. We shall refer to the irreducible components of the complement of $\mathscr{H C S}_{b, d}$ in $\mathscr{C} \mathscr{S}_{b, d}$ (respectively, .1 $\mathscr{S}_{b, d}$ ) as the "divisors at infinity" of $\mathscr{C} \mathscr{S}_{b, d}$ (respectively, $1 \mathscr{S}_{b, d}$ ). In this Section, we shall give an explicit construction as well as a complete combinatorial description of the "divisors at infinity" of $\mathscr{6} \mathscr{S}_{b . d}$. (One can then classify the divisors at infinity of $\mathscr{E} C_{b, d}$ according to which divisor at infinity of $\mathscr{E} \mathscr{S}_{b, d}$ they are mapped to; unfortunately, however, it is not clear that the irreducibility results for divisors at infinity of $\mathscr{E} \mathscr{S}_{b . d}$ extend to the divisors at infinity of $\mathscr{E}\left(\mathbb{C}_{b, d}\right.$.)

$\S 4.2$. Let $S$ be an $R$-scheme. Let $X_{t} \rightarrow S, i=1,2$ be prestable curves in the sense of [22], i.e., they are flat and proper and have geometric fibres that are reduced, of dimension one, and have at most ordinary double points as singularities. Let $\tau_{1}: S \rightarrow X_{1}$ be a section that lands in the smooth locus. By loc. cit, Theorem 3.4, p. 181, we can glue together the $X_{l}$ 's along the $\tau_{t}$ 's to obtain a new prestable curve $X \rightarrow S$. It is clear that this construction is functorial, so if $\left(Y_{l}, \rho_{l}\right)$ satisfy the same hypotheses as the $\left(X_{t}, \tau_{t}\right)$, then we obtain, by clutching, a new prestable curve $Y \rightarrow S$ as well as a morphism $X \rightarrow Y$ for every pair of morphisms $X_{t} \rightarrow Y_{l},(i=1,2)$ that carry $\tau_{1}$ to $\rho_{l}$. It thus follows that if we also give sections $\sigma_{1}^{(l)}, \ldots, \sigma_{b_{l}}^{(l)}(i=1,2): S \rightarrow Y_{1}$ that land in the smooth locus and are disjoint from each other and from $\rho_{l}$, in such a way that $\left(Y_{l}, \sigma_{1}^{(1)}, \ldots, \sigma_{b_{l}}^{(l)}, \rho_{l}\right),(i=$ $1,2)$ is a $\left(b_{t}+1\right)$-pointed stable curve, and if the morphisms $X_{t} \rightarrow Y_{l}$ satisfy all the conditions for an admissible covering of degree $d$ over $\left(Y_{t}, \sigma_{1}^{(t)}, \ldots, \sigma_{b_{1}}^{(t)}, \rho_{l}\right)$ except that: 
(1) the ramification of $X_{l} \rightarrow Y_{1}$ over $\rho_{1}$ might not be simple; however, it is tame, and, moreover, we give sections $\tau_{1}^{(l)}, \ldots, \tau_{l}^{(l)},(i=1,2): S \rightarrow X_{1}$ that land in the smooth locus, are disjoint from each other, map via $X_{1} \rightarrow Y_{1}$ to $\rho_{\imath}(i=1,2)$, and are such that over each geometric point $\bar{s}$ of $S$, $\tau_{l, \bar{s}}^{(l)} ; \ldots ; \tau_{l, \bar{s}}^{(l)}$ are exactly the points of $X_{l, \bar{s}}$ which are in the inverse image of $\rho_{t, \bar{s}}$ under $X_{t, \overline{5}} \rightarrow Y_{t, \overline{5}}$; moreover, the ramification index of $X_{t, \overline{5}} \rightarrow Y_{t, \overline{5}}$ at $\tau_{J, \bar{s}}^{(t)}($ for all $j$ ) is independent of $i$ (for $i=1,2$ );

(2) the fibres of $X_{t} \rightarrow S$ might not be geometrically connected, but if we glue together $X_{1}$ and $X_{2}$ along $\tau_{,}^{(1)}$ and $\tau_{J}^{(2)}$ (for all $j$ ), the resulting $X \rightarrow S$ has geometrically connected fibres;

then we may construct an admissible covering by taking for $X$ the gluing together of $X_{1}$ and $X_{2}$ along $\tau_{J}^{(1)}$ and $\tau_{j}^{(2)}$ (for all $j$ ) and for $Y$, the gluing together of $Y_{1}$ and $Y_{2}$ along $\rho_{1}$ and $\rho_{2}$, and for the map $X \rightarrow Y$, the functorially associated map for the pair of maps $X_{1} \rightarrow Y_{1}$ and $X_{2} \rightarrow Y_{2}$. In particular, note that $Y$ is necessarily stable.

Note that instead of gluing together two "almost admissible" coverings to obtain an admissible covering, we could just as well have glued together more than two "almost admissible" coverings (with the appropriate conditions analogous to those above on the data) to obtain an admissible covering. However, we shall have no need for this (only clerically) more difficult version of the above construction in this paper.

§4.3. Lemma. Let $S$ be an excellent Noetherian $R$-scheme. Let $V \rightarrow S$ be smooth of dimension 1 with section $\rho: S \rightarrow V$. Let $U \rightarrow S$ be smooth of dimension 1 with a finite, flat $S$-morphism $\varphi: U \rightarrow V$ which is étale except over $\rho$ where it has tame ramification. Let $W=$ the scheme $\varphi^{-1} S=U \times_{V} S$, where $S$ is a $V$ scheme via $\rho$. Then if $S$ is regular, $W_{\text {red }}$ is finite étale over $S$.

Proof. Using the excellence hypothesis (to ensure that taking "red" commutes with passing to completion - see EGA IV for a definition and basic facts about excellent rings), we reduce immediately to the case where $S=$ Spec $A$, where $A$ is a complete local ring with separably closed residue field. Let $s$ be the closed point of $S$. Then let $V^{\wedge}=$ the completion of $V$ at the prime $\rho(s)$. Let $U^{\wedge}=U \times_{V} V^{\wedge}$. Now $V^{\wedge}=\operatorname{Spec} A[[T]]$, where $T$ is an indeterminate such that the image subscheme of $\rho$ has ideal generated by $T$. By Abhyankar's lemma, $U^{\wedge}$ is the spectrum of a direct product of rings of the form $(A[[T]])[X] /\left(X^{n}-T\right)$ where $n$ is prime to char $k(s)$. The result follows. 0 
§4.4. Definitions. As in $\S 1$, we fix $b, d, g$. We define a layout $\mathscr{L}$ to be the following set of data:

(1) An ordered pair of integers $\left(i_{L}, i_{R}\right)$ with $i_{L}, i_{R} \geq 2, i_{L}+i_{R}=b$ (here $L$ stands for "left", $R$ for "right");

(2) Two ordered chains $C_{L}$ and $C_{R}$ of $i_{L}$ (resp. $i_{R}$ ) transpositions in $\mathscr{S}^{d}$ such that if we denote by $\Pi C_{L}$ (resp. $\Pi C_{R}$ ) the ordered product of the respective transpositions, then we have $\prod C_{L}=\prod C_{R}$; and such that the subgroup of $\mathscr{S}^{d}$ generated by all the transpositions of $C_{L}$ and $C_{R}$ acts transitively on $\{1, \ldots, d\}$.

When we need to deal with several layouts at once, we shall use notation like $i_{L}^{-\prime}$, $C_{L}^{\prime}$, etc. to denote the various objects associated to the layout $\mathscr{L}$. Let $D_{L}^{1}, \ldots, D_{L}^{n_{L}}$ (resp. $D_{R}^{1}, \ldots, D_{R}^{n_{R}}$ ) be the domains of transitivity (including those with only one element) of the group of permutations generated by the transpositions of $C_{L}$ (resp. $C_{R}$ ). We refer to these as the left (resp. right) domains of transitivity. The permutation $\Pi C_{L}=\prod C_{R}$ will be called the total clutching permutation $\sigma$. The ordering of $C_{L}$ gives us a natural bijection of $\left\{1, \ldots, i_{L}\right\}$ (resp. $\left\{1, \ldots, i_{R}\right\}$ ) with $C_{L}$ (resp. $\left.C_{R}\right)$. Relative to this bijection, let $B_{L}^{l}$ (resp. $\left.B_{R}^{t}\right)$, where $i=1, \ldots, n_{L}$ (resp. $i=1, \ldots, n_{R}$ ) be the subset of $\left\{1, \ldots, i_{L}\right\}$ (resp. $\left\{1, \ldots, i_{R}\right\}$ ) corresponding to those transpositions that act nontrivially on $D_{L}^{l}$. Let $b_{L}^{\prime}$ (resp. $\left.b_{R}^{l}\right)=$ the cardinality of $B_{L}^{l}$ (resp. $\left.B_{R}^{\prime}\right)$. We call $b_{L}^{\prime}$ (resp. $\left.b_{R}^{\prime}\right)$ the $i^{\text {th }}$ left (resp. right) partition number of the layout. The permutation which is the ordered product of all the transpositions of $C_{L}$ (resp. $C_{R}$ ) that correspond to elements of $B_{L}^{\prime}$ (resp. $B_{R}^{\prime}$ ) will be called the $i^{\text {th }}$ left (resp. right) clutching permutation $\sigma_{L}^{\prime}$ (resp. $\sigma_{R}^{\prime}$ ) and will also be written $\prod_{D_{L}^{\prime}} C_{L}$ (resp. $\prod_{D_{R}^{\prime}} C_{R}$ ). Note that the total clutching permutation $\sigma$ is the product of the $\sigma_{L}^{l}$ (resp. $\sigma_{R}^{l}$ ) over $i=1, \ldots, n_{L}$ (resp. $\left.i=1, \ldots, n_{R}\right)$. Two layouts will be said to be isomorphic if one can be obtained from the other by switching left and right and/or by conjugating all the data by a some element of $\mathscr{F}^{d}$. It is clear that isomorphism is an equivalence relation. We denote the (finite) set of layouts by $\mathbb{L}_{b . d}$ (or simply $\mathbb{L}$ when $b, d$ are fixed and clear) and the set of isomorphism classes of layouts by $\mathbb{C L}_{b . d}$ (or simply $\left.\mathbb{C L}\right)$.

Given a layout $\mathscr{L}^{\prime}$, we define its template $\mathcal{T}$ to be the following set of data:

(1) The ordered pair $\left(i_{L}, i_{R}\right)$; 
(2) The (unordered) set of "left" (resp. "right") triples of the following sort: Each "left" (resp. "right") triple consists of a left (resp. right) domain of transitivity, its associated clutching permutation, and its associated partition number.

Let $\mathbb{T}_{b, d}$ (or simply $\mathbb{T}$ ) be the set of templates $\mathscr{T}$ corresponding to all possible layouts $\mathscr{L}$. Note that we have an analogous notion of isomorphism of templates, corresponding to switching left and right and/or conjugating by a permutation. Let $\mathbb{C T}_{b . d}$ (or simply $\mathbb{C} \mathbb{T}$ ) be the set of isomorphism classes of templates. Then we have the following natural surjections:

$$
\begin{aligned}
& \Phi: \mathbb{L} \rightarrow \mathbb{T} \\
& \Psi: \mathbb{C L} \rightarrow \mathbb{C T}
\end{aligned}
$$

\$4.5. Theorem. The divisors at infinity of $\mathscr{G} \mathcal{S}_{\text {b.d }}$ are in natural one-to-one correspondence with the elements of $\mathbb{C} \mathbb{T}$.

Proof. The proof will consist of several steps:

\section{Step A. Construction of divisors at infinity:}

Let $\mathscr{T} \in \mathbb{T}_{b, d}$ be a template. We start by constructing the "left $i_{L}$-point stack". (Of course, the construction of the right $i_{R}$-point stack is entirely analogous.) Let $\mathscr{Z} \mathscr{K} C^{\prime}=\mathscr{R} C_{\left(L_{L}+1\right)}$, where $\mathscr{L}$ stands for "left", $\mathscr{R} C$ for "rigidified, ordered" (as usual), and $\mathscr{T}$ denotes the dependence on the template. Let $b_{1}, \ldots, b_{n_{L}}$ be the left partition numbers (in any order!) of the template $\mathcal{T}$. Let us partition the set $\left\{1, \ldots, i_{L}\right\}$ into disjoint subsets $A_{t}$ (where $i=1, \ldots, n_{L}$ ) of $b_{\text {l }}$

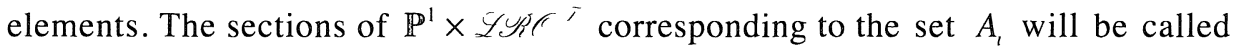
sections of the $i^{\text {th }}$ partition. The last section will be called the clutching section. Let $\pi_{1}$ : LYY $\rightarrow$ GYY $\mathrm{C}_{b_{1}+1}$ denote the map corresponding to sending the sections of the $i^{\text {th }}$ left partition (in some order) to the marking sections of $\mathscr{M} \mathscr{K} C_{b_{1}+1}$ and the clutching section to the clutching section (recall the terminology from $\S 1$ ). Now $G=\mathscr{f}^{b_{1}} \times \mathscr{Y}^{b_{2}} \times \ldots \times \mathcal{J}^{b_{n_{L}}}$ generically acts freely on $\mathscr{L}_{R} \mathcal{C}^{\prime}$ by allowing the $i^{t^{t h}}$ factor to act naturally on the sections of the $i^{\text {th }}$ partition. We define the left $i_{L^{-}}$ point stack, $\mathcal{Y K Y}^{\prime} \mathcal{Y}^{\prime}$, to be the quotient (in the sense of stacks) of $\mathcal{Y Y R C}^{\prime}$ by $G$, which is generically a scheme (by the generic freeness of the section of $G$ ). Note that the $\pi_{1}$ 's induce natural projections $\varphi_{1}: \mathscr{L Y K}^{\prime} \mathcal{S}^{\prime} \rightarrow \mathscr{Y Y K F}_{b_{1}+1}$.

Now let $\sigma_{1} \in \mathscr{Y}^{d}$ be the $i^{\text {th }}$ left clutching permutation. Let $D_{1}$ be the $i^{\text {th }}$ left domain of transitivity, and let $d_{1}=$ the cardinality of $D_{1}$. Then we constructed $(\$ 1.4,1.5) \mathscr{T} \mathscr{H} \mathscr{R} \mathcal{F}_{b_{1}+1 . d_{t}}^{\sigma_{t}}$ and a "pseudo-universal" covering: 


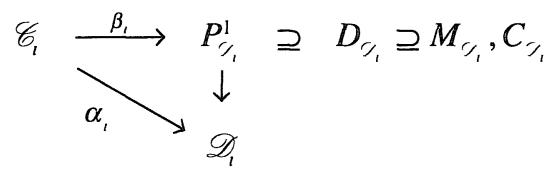

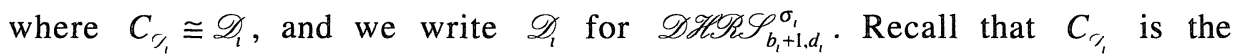
clutching section, while $M_{r_{1}}$ (which is finite étale of rank $b_{l}$ over $\mathscr{D}_{1}$ ) is the marking divisor. Since $\mathscr{D}_{1}$ is regular, it follows by Lemma 3 that the reduced inverse image via $\beta_{1}$ of $C_{1 / 1}$ is étale over $C_{1 / 1} \cong \mathscr{D}_{1}$; let us denote this reduced inverse image by $C_{\tilde{j}_{i}}$. Now the question arises: is $C_{\tilde{j}_{1}}$ "geometric" over $R$ in the sense that the étale covering $C_{\tilde{z}_{1}} \rightarrow C_{r_{1}}$ does not factor through a covering obtained by tensoring up by an étale cover $R^{\sim}$ of $R$ ? I claim that the answer is "yes". Indeed, recall from Proposition 10 of $\$ 2$ that the connected components over $R$ correspond naturally to the various ramification indices, and these components are, in fact, geometric over $R$.

We are now in a position to construct the left degenerate Hurwitz stack associated to the template $\mathscr{T}$, which we shall call $\mathscr{L} \mathscr{D} \mathscr{H}^{\top}$. We define $\mathscr{L} \mathscr{D} \mathscr{H}^{\top}$

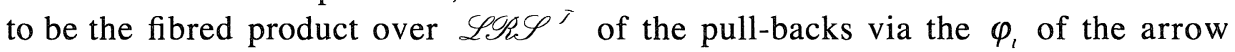
$\mathscr{D}_{1} \rightarrow \mathscr{D} \mathscr{R} \mathscr{S}_{b_{1}+1}$. It is clear by the results of $\$ 2$ that $\mathscr{L} \mathscr{D} \mathscr{H}^{\prime}$ is smooth and geometrically irreducible over $R$. We can also pull-back the coverings $C_{i_{1}} \rightarrow \mathscr{D}_{1}$

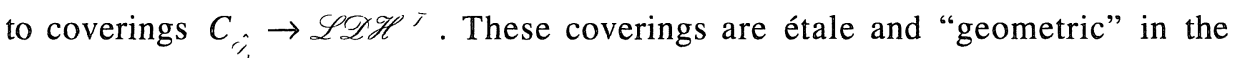
sense used above. Therefore, by basic facts of Galois theory, there exists a (noncanonical) finite étale Galois covering $\Lambda \rightarrow \mathscr{L} \mathscr{D} \mathscr{H}^{\top}$ that dominates all the connected components of all the $C_{i_{i}}$ (for all $i$ ) and which is geometrically irreducible over $R$. We denote the pull-backs of the objects $\mathscr{E}_{1}, \alpha_{1}, \beta_{1}, M_{r_{1}}, C_{r_{1}}$, $C_{i_{i}}, D_{1,}$ by $\mathscr{C}_{1}^{\Lambda}, \gamma_{1}, \delta_{l}, M_{1}^{\Lambda}, C_{1}^{\Lambda}, C_{1}^{\sim \Lambda}, D_{1}^{\Lambda}$. We thus obtain a commutative diagram:

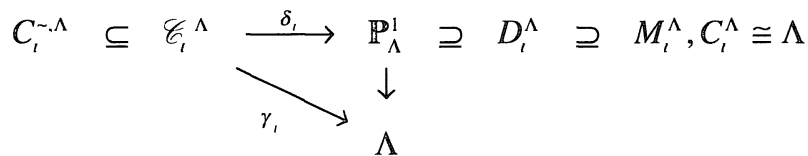

(Diagram 4.1)

where the relative dimension of $\Lambda$ is $i_{L}-2$. Note that $C_{1}^{\sim . \Lambda} \rightarrow \Lambda$ is split (i.e., every connected component of $C_{l}^{\sim, \Lambda}$ maps isomorphically to $\Lambda$ ).

Analogously, we construct the right degenerate Hurwitz stack $\mathscr{R} \mathscr{D} \mathscr{H}^{\top}$ and, in place of $\Lambda$, we have $P$ (the capital Greek letter "rho"). We thus obtain a diagram: 


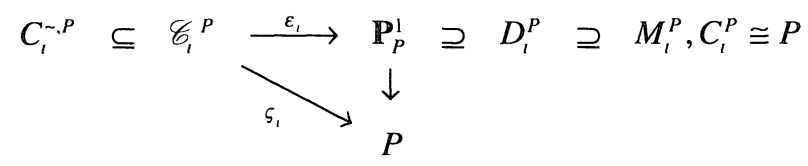

(Diagram 4.2)

where (again) the relative dimension of $P$ over $R$ is $i_{R}-2$. Thus $\mathscr{K} \stackrel{\text { det }}{=} \Lambda \times_{R} P$ has relative dimension over $R$ equal to $b-4$. This is just the right dimension for a divisor of $\mathscr{C S}_{b, d}$.

Let us pull the Diagrams 4.1 and 4.2 back to $\mathscr{K}$ via the projections to $\Lambda$ and $P$, and denote the objects so obtained by $C_{l, \tilde{H}}^{\sim, \Lambda} ; C_{l, \grave{r}}^{\Lambda}$; etc. Now we first glue the left and right copies of $\mathbb{P}^{1}$ together along $C_{l}^{\Lambda}$ and $C_{l}^{P}$; using the marking divisors $M_{l}^{\Lambda}$ and $M_{l}^{P}$ we thus obtain a $b$-pointed stable curve $B \rightarrow \mathscr{K}$. Let us next note that (referring to $\$ 4.4$ ) the irreducible components of $C_{1}^{\Lambda}$ (resp. $C_{1}^{P}$ ) correspond naturally to the left (respectively, right) domains of transitivity, which is part of the information of the layout that is preserved in the template. Thus, there is a natural isomorphism $C_{l}^{\Lambda} \cong C_{l}^{P}$. Let us glue together $\coprod_{l=1, n_{L}} \mathscr{C}_{l, / 7}^{\Lambda}$ and $\coprod_{l=1,, n_{R}} \mathscr{C}_{l, \text { / }}^{P}$ (both prestable curves) along $C_{l}^{\Lambda}$ and $C_{l}^{P}$ via this isomorphism and call the resulting curve $X \rightarrow \mathscr{K}$. It follows from the conditions in the definition of a layout and by what we did in $\$ 4.2$, that we then obtain an admissible covering $f: X \rightarrow B$ over $\mathscr{K}$. By the modular definition of $\mathscr{E} \mathscr{S}_{b . d}$, we then obtain a morphism $\mathscr{K} \rightarrow \mathscr{C} \mathscr{S}_{b, d}$ which we call $M^{\top}$. It is clear by composition with the morphism $\mathscr{G} \mathscr{S}_{b, d} \rightarrow \overline{\mathscr{R} S}_{b}$ that $M^{\top}$ is quasi-finite. Thus, for dimensional reasons, the closure of its image must be an irreducible divisor of $\mathscr{C}_{b, d}$, which is flat over $R$. We shall refer to this divisor as the divisor $D^{\prime}$ associated to $\mathscr{T}$. We shall also refer to any divisor of $.1 \mathscr{S}_{b, d}$ that lies above $D^{\zeta}$ as a "divisor associated to $\mathscr{T}$ ". Finally, it is clear that isomorphic templates define the same divisor. Thus we obtain a map:

$$
\Omega: \mathbb{C T} \rightarrow\left\{\text { divisors at infinity of } \mathscr{C} \mathscr{S}_{b, d}\right. \text { \} }
$$

\section{Step B. Surjectivity of $\Omega$ :}

Since $\mathscr{G} \mathscr{S}_{b, d} \rightarrow \overline{\mathscr{R} F}_{b}$ is flat (Corollary 23 of $\S 3$ ), every divisor at infinity of $\mathscr{C} \mathscr{P}_{b, d}$ lies above a divisor at infinity of $\mathscr{R} \mathscr{S}_{b, d}$, whose generic element is necessarily of the form given in Diagram 3.1. Thus it suffices to see that every geometric point of $\mathscr{C} \mathscr{S}_{b, d}$ such that the stable curve downstairs is of the form given in Diagram 3.1 (in the future, we shall call such a covering a basic 
admissible covering of type $(i, j)$, where $(i, j)$ is as in loc. cit.) is in the image of a geometric point of one the $M^{\top}$ 's. But this is obvious from the construction.

\section{Step $\mathbb{C}$. Injectivity of $\Omega$ :}

It suffices to see that given any basic admissible covering, we can recover the information contained in a template from the purely geometric data of the covering. But this is clear, for left (resp. right) domains of transitivity correspond to the irreducible components on the left (resp. right) hand side of the curve upstairs, associated clutching permutation correspond to the ramification data at clutching points, and partition numbers correspond to the number simple ramification points of each irreducible component upstairs. $O$

\$4.6. Any particular divisor at infinity of $\mathscr{6} \mathscr{F}_{b, d}$ will be called a Species. If $\left(i_{L}, i_{R}\right)$ is the first piece of data of a template $\mathscr{T}$ (see $\S 4.4$ ), then we call $\mathscr{T}$ a "template of type $\left(i_{L}, i_{R}\right)$ ". We also say that the corresponding Species is of type $\left(i_{L}, i_{R}\right)$. Before going on, it is worthwhile to enumerate the various Species of type $(b-2,2)$. By the previous Theorem, this amounts to classifying explicitly the various templates that appear for basic admissible coverings of type $(b-2,2)$.

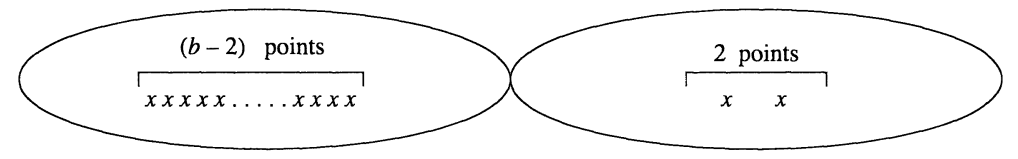

Let $C$ be a component on the right hand side of the curve upstairs for such a covering. Then either:

I. $C$ has no "x"'s (i.e., simple ramification points); then $C$ maps isomorphically to the copy of $\mathbb{P}^{1}$ downstairs;

II. $C$ has exactly one "x"; then it is clear that $C \cong \mathbb{P}^{1}$ and the map downstairs must be the "squaring map"; there is one clutching point on $C$, where the ramification index is two; or

III. $C$ has exactly two "x"'s; then each of those corresponds to a transposition; up to renaming elements, these two transpositions are either:

(A) (12), (23): then $C \cong \mathbb{P}^{1}$; there is one clutching point on $C$ with ramification index three; the map downstairs is some uniquely determined map; 
(B) (12), (12): then $C \cong \mathbb{P}^{1}$; there is no ramification at the clutching points, of which there are two on $C$; the map downstairs is the "squaring map";

(C) (12), (34): the degree of the map from $C$ to the copy of $\mathbb{P}^{1}$ downstairs would have to be four, and the degree of the total ramification divisor on $C$ would also have to be four; thus this case is impossible by the Riemann-Hurwitz formula.

Let us now consider the totality of components on the right. Exactly one of the following two possibilities must hold:

(1) There are two components on the right with one " $x$ " each and all the other components have no " $x$ "'s. In this case, let us consider the associated graph of the curve upstairs. On the right, we have $d-2$ vertices, each with exactly one edge protruding from it. It follows that if the graph is to be connected (which it must be), then there can only be one irreducible component on the left. This component is a smooth component of genus $g$ (the original genus) and has $d-2$ clutching points, one going to each irreducible component on the right. Of these, there is ramification of degree 2 at two clutching points (which we shall call points of degenerate ramification), and no ramification at any of the $d-4$ others. It is thus clear that in this case, the template is uniquely determined. We call the corresponding divisor at infinity the divisor of Species 2. A typical basic admissible covering of this Species is shown in the Pictorial Appendix.

(2) There is exactly one component on the right with two "x"s, and all the other components have no " $x$ "'s. Then the component with two " $x$ "' $s$ is as in exactly one of the following, corresponding, respectively, to the Cases (A) and $(B)$ of the preceding III:

(A) It is clear that the graph of the curve upstairs is the same in (1) above. Thus there is only one irreducible component on the left; it is smooth of genus $g$ and has $d-2$ clutching points, one going to each irreducible component on the right. Of these, there is ramification of degree 3 at one clutching point (which we shall call the point of degenerate ramification) and no ramification at any of the $d-3$ others. It is thus clear that in this case, the template is uniquely determined. We call the corresponding divisor at infinity the divisor of Species 1. A typical basic admissible covering of this Species is shown in the Pictorial Appendix. 
(B) Let us consider the graph associated the curve upstairs. On the right, we have $d-1$ vertices; from $d-2$ of them, there protrudes exactly one edge; from the other one of them there protrude exactly two edges. Now either those two edges end up at the same vertex on the left, or they end up at different vertices. We thus examine each of these two cases:

$\alpha)$ The Same Vertex: Then there is only one vertex on the left. This corresponds to a smooth, irreducible component of genus $g-1$, since the loop on the graph contributes "one" to the arithmetic genus of the curve upstairs. There are exactly $d$ clutching points on this component on the left, all of which are unramified.

Of these $d-2$ connect to components on the right of type (I) in our classification above of components of the curve upstairs, and the other 2 (which we shall points of degenerate ramification) connect to the component of type (III) (B) in that classification. Clearly the template is thus uniquely determined. We call the corresponding divisor at infinity the divisor of Species 3A. A typical basic admissible covering of this Species is shown in the Pictorial Appendix.

$\beta$ ) Different Vertices: Then it is clear that these are the only two vertices on the left. They correspond to two smooth, irreducible components $C_{1}$ and $C_{2}$. Let $g_{1}$ be the genus of $C_{1}$ (where $i=1,2$ ). If $b_{l}=$ the number of "x"'s on $C_{1}$, and $d_{l}$ is the degree of $C_{1}$ over the left hand copy of $\mathbb{P}^{1}$, then we have, by the Hurwitz formula, $2\left(g_{1}-1\right)+2 d_{1}=b_{1}, \quad g_{1}+g_{2}=g, \quad b_{1}+b_{2}=b-2, \quad d_{1}+d_{2}=d$. The map downstairs $C_{1} \rightarrow \mathbb{P}^{1}$ thus has only simple ramification. The clutching points are all unramified with one clutching point on each $C_{t}$ (which we shall refer to as a point of degenerate ramification) going to the component of type (III) (B); the other $d_{t}-1$ clutching points on each $C_{t}$ go to components of type (I). Given pairs of integers $\left(b_{1}, b_{2}\right),\left(d_{1}, d_{2}\right)$ such that $b_{1}+b_{2}=b-2$, $d_{1}+d_{2}=d$, with $b_{1} \geq 0, d_{1} \geq 1$ and such that the $g_{1}$ 's computed via the Hurwitz formula are nonnegative integers (for $i=1,2$ ) will always appear, and once the $b_{l}$ 's and $d_{l}$ 's are given, the template is clearly uniquely determined. The corresponding divisor at infinity will be call the divisor of Species $3 B\left(b_{1}, b_{2}\right) ;\left(d_{1}, d_{2}\right)$. It will turn out that the case when $b_{1}=b-2, b_{2}=0, d_{1}=d-1$, $d_{2}=1 \quad\left(\Rightarrow g_{1}=g, g_{2}=0\right)$ will be especially important; we shall 
call this case the divisor of Species $3 B^{*}$. As usual, illustrations are shown in the Pictorial Appendix.

$\$ 4.7$. Let us note that among the templates of type $(b-2,2)$, the Species that do not map via the map $\mathscr{E} \mathscr{S}_{b . d} \rightarrow \overline{\mathscr{M}}_{g}$ of $\$ 3.27$, into the boundary at infinity of $\overline{\mathscr{M}}_{g}$ are exactly Species 1,2 , and $3 B\left(b_{1}, b_{2}\right) ;\left(d_{1}, d_{2}\right)$ when $g_{1}=g, g_{2}=0$. Among these, it turns out that the divisors of Species 1,2 , and $3 B^{*}$ (which constitutes a set of precisely three divisors) will play an important role in the future. We shall refer to these three divisors as the excess divisors.

$\S 4.8$. If $F$ is a ring and $\mathbb{E}$ is a set, we shall denote by $F[\mathbf{E}]$ the free $F$-module generated by elements of $\mathbb{E}$. By Theorem 5 , to every element of the set $\mathbf{C T}$, one can naturally associate a divisor, hence a divisor class of $\mathscr{C} \mathscr{S}_{b, d}$, i.e., an element of $C l\left(\mathscr{C S}_{b . d}\right)$. We thus obtain a map:

$$
\Sigma: \mathbb{Z}[\mathbf{C T}] \rightarrow C l\left(\mathscr{E} \mathcal{S}_{b, d}\right)
$$

Later we shall show the following

Theorem. If $d \geq 2 g+4, g \geq 5$, then the group $C l\left(\mathscr{G} \mathscr{S}_{b . d}\right)$ is of finite type over $\mathbb{Z}$, and $\sum$ is a surjection when tensored with $\mathbf{Q}$.

Clearly it suffices to show that $\operatorname{Pic}\left(\operatorname{HYMS}_{b, d}\right)$ is finite. We shall complete the proof in $\$ 6.7$.

\section{Appendix to $\$ 4$}

§4.9. In SGA 7, one finds an exposition of the theory of vanishing cycles for ordinary (i.e., 0-pointed) stable curves. Although the theory of vanishing cycles for arbitrary $b$-pointed stable curves is also implicit in SGA, we review it here explicitly for the sake of clarity. In the case of $b$-pointed stable curves of genus zero, which is the principal case of interest here, one can often describe the monodromy action much more explicitly than in the 0 -pointed higher genus case since the fundamental group involved is a free group.

$\S 4.10$. Let $(A, m)$ be a strictly henselian discrete valuation ring, $S=\operatorname{Spec} A$. Let $\left\{C \stackrel{t}{\longrightarrow} S ; \sigma_{1}, \ldots, \sigma_{b}: S \rightarrow C\right\}$ be a $b$-pointed stable curve over $S$. Let $U=C$ $-\bigcup_{i=1}^{b} \sigma_{1}(S)$. We use the notation $U_{\eta}, U_{\bar{\eta}}, U_{1}$, as usual. Then we have the following

Proposition. There is a natural, surjective morphism of specialization: 


$$
\pi_{1}^{t}\left(U_{\bar{\eta}}, \rho_{\bar{\eta}}\right)^{\prime} \rightarrow \pi_{1}^{t}\left(U_{s}, \rho_{s}\right)^{\prime}
$$

where $\rho: S \rightarrow U$ is a section of $f$, and " $t$ " means the tame fundamental group, and the "prime" next to the fundamental group indicates that we are only concerned with the part of the fundamental dealing with coverings of degree prime to the residue characteristic of $A$.

Remark. It has been pointed out by the referee that this result has already been proven by Grothendieck in [5].

Proof. Write $j: U \rightarrow C$ for the natural inclusion. Let $G=$ a finite group of order prime to the residue characteristic of $A$. By [29], Exposé XIII, Theorem 2.4 , p. 380, we have $\mathbb{R}_{e t}^{1} j_{s},\left.G_{U} \cong\left(\mathbb{R}_{e t}^{1} j G_{U}\right)\right|_{C_{1}}$. By the Leray spectral sequence and the proper base change theorem, we thus see that $\pi_{1}^{t}\left(U_{s}, \rho_{\mathrm{s}}\right) \cong \pi_{1}^{t}\left(U, \rho_{\mathrm{s}}\right) \cong$ $\pi_{1}^{t}\left(U, \rho_{\bar{\eta}}\right)$. The rest of the Proposition then follows trivially. $\bigcirc$

$\$ 4.11$. Let us now assume that $C$ is obtained in the following fashion. We are given a collection of generically distinct sections $\sigma_{1}^{\prime}, \ldots, \sigma_{b}^{\prime}: S \rightarrow \mathbb{P}_{S}^{1}$ such that $\sigma_{1}^{\prime}(S)$ intersects $\sigma_{2}^{\prime}(S)$, but all the other intersections among pairs of $\sigma_{1}^{\prime}$ are empty. Then $C$ is obtained by blowing up $\mathbb{P}_{S}^{1}$ at the schematic intersection of $\sigma_{1}^{\prime}(S)$ and $\sigma_{2}^{\prime}(S)$; we thus obtain a stable curve $\left\{C \stackrel{f}{\longrightarrow} S ; \sigma_{1}, \ldots, \sigma_{b}\right\}$ where the $\sigma_{t}$ are induced by the $\sigma_{1}^{\prime}$. Now if we fix a base section $\rho$ (that doesn't intersect any of the $\sigma_{l}$ ), and assume (for simplicity) that we are working over $\mathbb{C}$, then $\pi_{1}^{a n}\left(U_{\bar{\eta}}, \rho_{\bar{\eta}}\right)$ may be described as being generated by paths $\gamma_{1}, \ldots, \gamma_{b}$ around the various $\sigma_{1, \bar{\eta}}$ with the sole relation that $\gamma_{1} \gamma_{2} \ldots \gamma_{b}=1$, i.e., a free group on $b-1$ generators. On the other hand, $\pi_{1}^{a n}\left(U_{s}\right)$ may be described as being generated by paths $\delta_{1}, \ldots, \delta_{b}$ around the various $\sigma_{t,}$ with the sole relations $\delta_{1} \delta_{2}=1, \delta_{3} \delta_{4} \ldots \delta_{b}$ $=1$. An illustration of this degeneration situation is given in the Pictorial Appendix. The morphism of specialization is given by $\gamma_{1} \mapsto \delta_{l}$, so the "vanishing cycle" is clearly $\gamma_{1} \gamma_{2}=\left(\gamma_{3} \gamma_{4} \ldots \gamma_{b}\right)^{-1}$. 


\section{Pictorial Appendix}

\section{Species 1:}

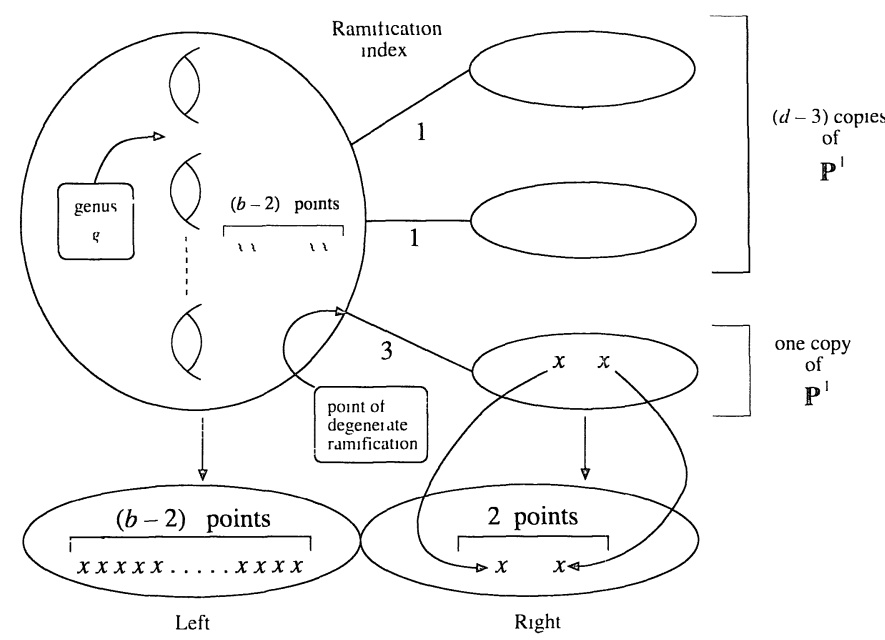

Species 2:

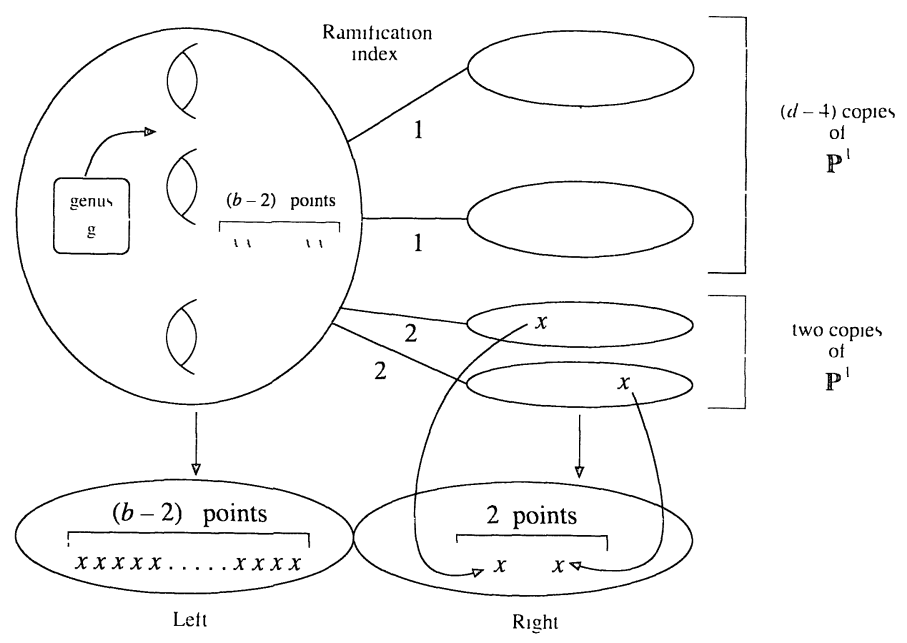




\section{Species $3 A$ :}

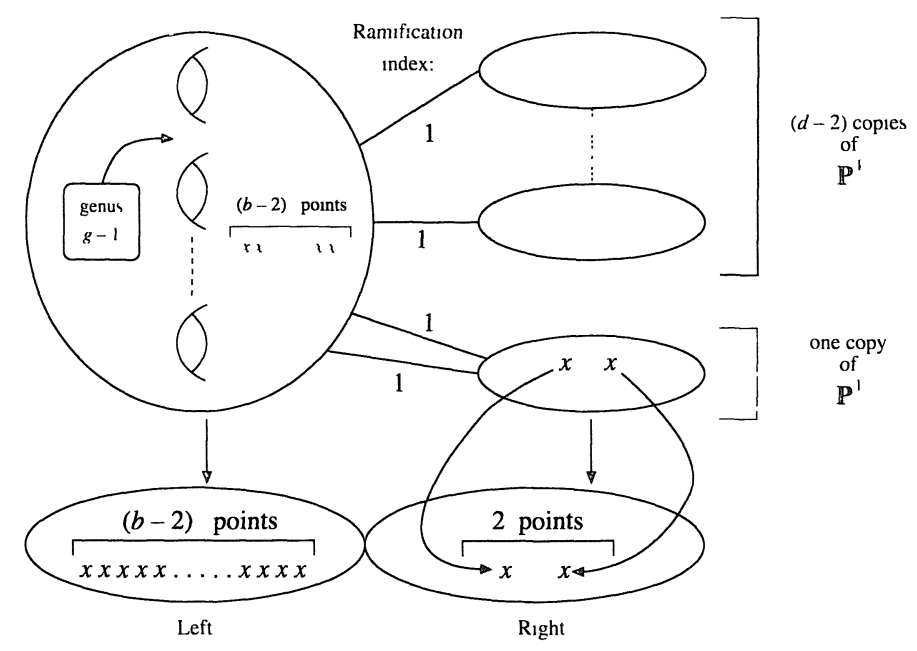

Species $3 B\left(b_{1}, b_{2}\right) ;\left(d_{1}, d_{2}\right)$ :

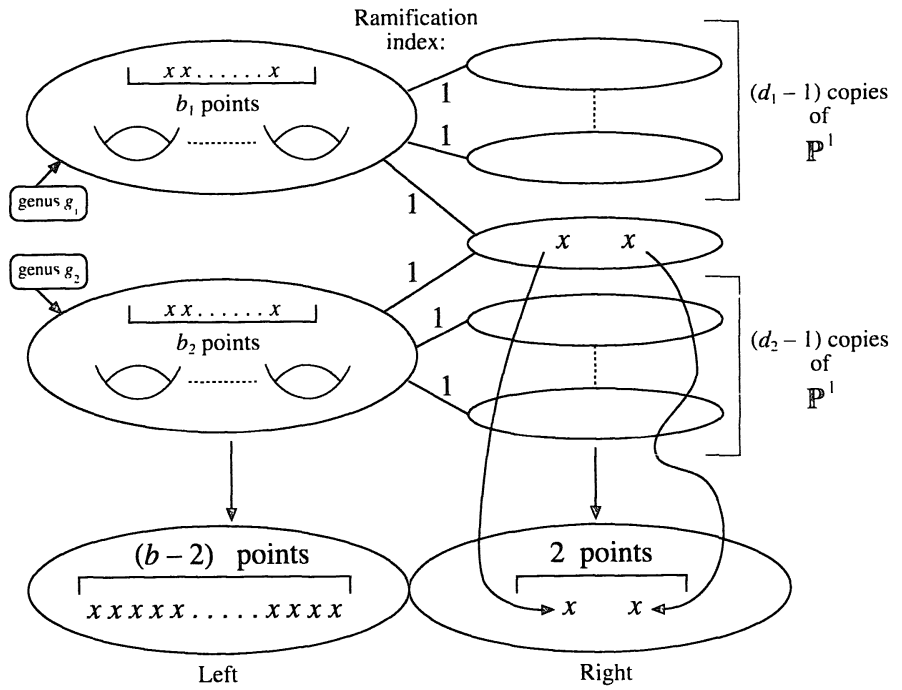


Species $3 B^{*}$ :

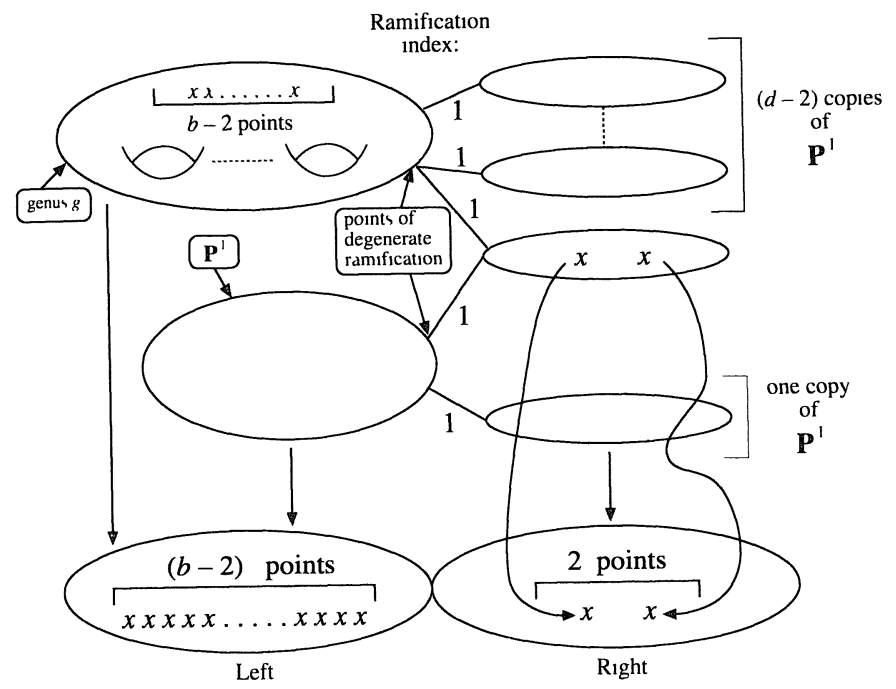

Degeneration Situation with Vanishing Cycle:

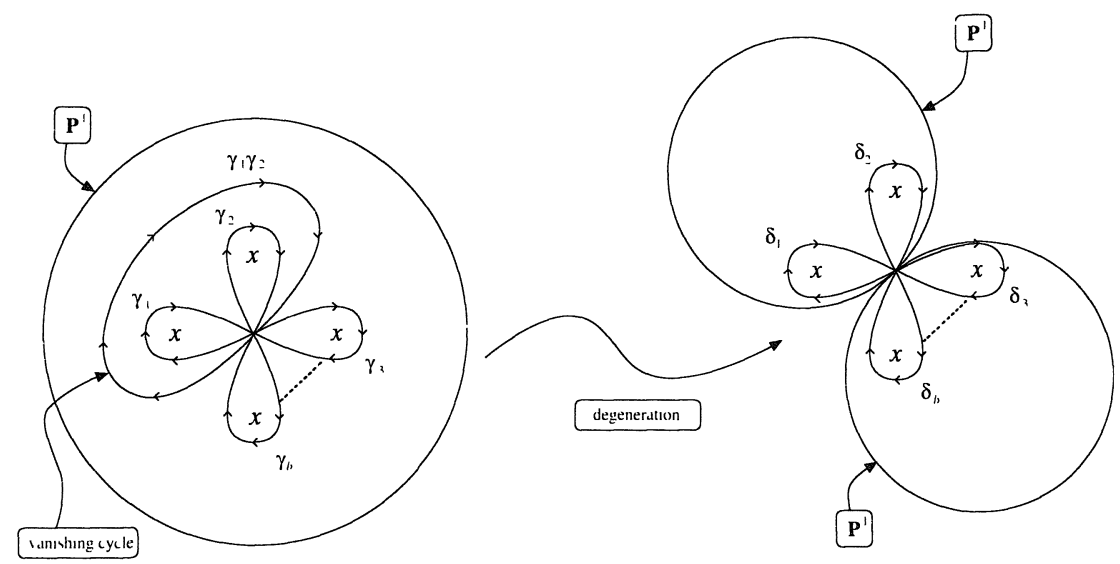

§. Cohomology Calculations

$\S 5.1$. In this Section, we compute the various cohomology groups of a number of universal objects, in preparation for the construction of the "main fibration" in the next Section. At first, we shall work over $\mathbf{C}$, so that we can use a number of analytic results; at the end of the Section, we shall extend the results to the 
arithmetic situation over $\mathbb{Z}$. Thus, unless we specify otherwise, all objects are taken to be over the complex numbers $\mathbb{C}$.

Often in this Section, we shall use the language of torsors over a group; for the reader unfamiliar with this language, we suggest [15] as a place to start.

$\S 5.2$. Let $\mathscr{M}_{g}=$ the moduli stack of smooth curves of genus $g$ with $g \geq 5$. Let $f: \mathscr{C} \rightarrow \mathbb{N}_{g}$ be the universal curve of genus $g$. Let $\mathscr{P}=\operatorname{Pic}\left(\mathscr{C} / \mathscr{M}_{g}\right)$ be the Picard stack, a smooth algebraic group stack over $\mathscr{M}_{g}$, which doesn't necessarily represent the Picard functor Pic (since $f$ doesn't admit a section), but is, instead, its étale sheafification (see, e.g., [12] for a detailed discussion of the Picard functor); thus we have a morphism Pic $\rightarrow \mathscr{P}$. Let $\mathscr{J}=\operatorname{Pic}^{\tau}\left(\mathscr{C} / \mathscr{M}_{g}\right) \stackrel{\varphi}{\longrightarrow} \mathscr{M}_{g}$ be the open subgroup which is the universal Jacobian. Let $\mathscr{E}=\mathscr{P} / \mathscr{J}$ be the quotient étale group stack, with geometric fibres over $\mathscr{l}_{g}$ isomorphic $\mathbb{Z}$. Thus we have the following situation:

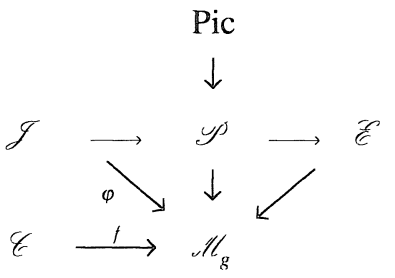

Now from the existence of the global bundle $\omega=\Omega_{,}^{\prime}$, ", on " $\%$, or by the existence of the degree map, one sees that $\mathscr{E} \cong \mathbb{I}_{\mathrm{q}} \times \mathbb{Z}$. Thus $\mathscr{P}$ splits into connected components $g^{d}$, with $d \in \mathbb{Z}$, according to the degree. Each $g^{d}$ is thus $\mathscr{g}$ torsor in the étale topology over. $\mathbb{I}_{g}$, i.e., an element $\eta_{d} \in H_{1}^{1}\left(, \|_{g}, \mathscr{J}\right)$. From the fact that $\mathscr{\rho}$ is a group stack, we see that $\eta_{d}=d \eta_{1}$. From the existence of $\omega$, we see that $2(g-1) \eta_{1}=0$. Let $\chi=2(g-1)$. Then if we "change groups" for the torsor $\mathscr{J}^{d}$ via the homomorphism $[\chi]: \mathscr{J} \rightarrow \mathcal{J}$ multiplication by $\chi$, we obtain a new $g$-torsor $g^{-d}$, which is clearly trivial. Moreover, by the Leray spectral sequence, we see that $H_{c m}^{\prime}\left(\mathscr{Y}^{d}, A\right) \cong H_{c m}^{\prime}\left(\mathscr{Z}^{-d}, A\right) \cong H_{c m}^{\prime}(\mathscr{J}, A)$ (where "an" denotes the analytic topology) for any ring $A$ in which $\chi$ is invertible.

$\S 5.3$. We recall the following results of [16] and [1]:

(A) Pic $\|_{q} \cong H_{c m}^{2}\left(\|_{p}, \mathbb{Z}\right) \cong \mathbb{Z}$. where the first isomorphism is the first Chern class map, and Pic "/, is generated by the Hodge bundle $\lambda$.

(B) Pic $₹ \cong H_{\text {anl }}^{2}(₹, \mathbb{Z}) \cong \mathbb{Z}^{2}$, where the first isomorphism is the first Chern class map, and Pic $₹$ is generated by $\lambda$ and $\omega$. 
(C) Let $\overline{\mathscr{F}}=\mathbb{R}_{a n}^{1} f \mathbb{Z} \cong \mathbb{R}_{a n}^{1} \varphi, \mathbb{Z}$. Then $H_{a n}^{1}\left(\|_{\mathrm{g}}, \tilde{F}\right)$ vanishes. (In fact, in [16], Harer makes the stronger assertion than in fact the first homology group of $\mathscr{F}$ vanishes; later in a correction (whose existence is mentioned in [17], Chapter 7) he retracts this and asserts only that the first homology group of $\mathscr{F}$ is torsion; but this implies that the first cohomology group must vanish.)

(D) Pic $\left(\mathscr{E} \times{ }_{14} \& \times \ldots \times \mathscr{E}\right)$ is finitely generated for any number of factors of $\mathscr{E}$. (In fact, the result in [1] is stated only for the moduli stack of $b$ pointed stable curves of genus $g$; but one deduces (D) easily from this fact using elementary properties of the Picard group.)

Recall that $\mathscr{F}$ is a locally constant sheaf of free $\mathbb{Z}$-modules of rank $2 g$ on $/ / g$. Moreover, $\mathcal{F}$ is endowed with a natural symplectic form $E$ ("the intersection pairing").

Lemma。 $H_{a n}^{0}\left(, \|_{\mathrm{p}}, \mathbb{R}_{\text {cm }}^{2} \varphi, \mathbb{Z}\right) \cong \mathbb{Z}$ and is generated by the element corresponding to E under the isomorphism $\mathbb{R}_{\text {an }}^{2} \varphi \mathbb{Z} \cong \wedge^{2} \cdot \mathscr{F}$.

Proof. Since $E$ is not a multiple of any other form, it suffices to prove the result after tensoring with $\mathbb{Q}$. If $M$ is the $\Gamma$-module (where $\Gamma=\pi_{1}^{u \prime \prime}\left(\mathscr{A}_{\mathrm{q}}\right)$ ) corresponding to $\overline{\mathcal{T}} \otimes_{\mathrm{Z}} \mathbb{Q}$, then since $\wedge_{\mathbf{Q}} M \subseteq M \otimes_{\mathrm{Q}} M \cong \operatorname{Hom}_{\mathbf{Q}}(M, M)$ (where the last isomorphism of $\Gamma$-modules follows from the fact that via $E, M$ is a selfdual $\Gamma$-module), it suffices to show that $M$ is an irreducible $\Gamma$-module. But this action factors through the surjection [8], p. 107, $\Gamma \rightarrow \operatorname{Sp}(2 g, \mathbb{Z})$, and one sees from looking at well-known sets of generators that $\operatorname{Sp}(2 g, \mathbb{Z})$ is Zariski dense in $\operatorname{Sp}(2 g, \mathbb{Q})$. Moreover, it is obvious that $M$ is irreducible under the action of $\operatorname{Sp}(2 g, \mathbb{Q})$. The Lemma follows. O

\$5.4. Proposition. There exists an isomorphism . : J $\longrightarrow \mathcal{J}^{\wedge}$ (i.e., the dual abelian stack to $g$ ) whose corresponding alternating form is $E$.

Proof. This, in fact, holds over $\mathbb{Z}$, so for the remainder of $\$ 5.4$, we work over $\mathbb{Z}$, not $\mathbb{C}$. The Proposition is well-known when there exists a section of $\sharp$. Thus, let $K=$ the quotient field of $/_{g}$; let $L$ be a finite Galois extension of $K$ such that $\bar{Z}_{L}$ admits a section. We thus obtain an isomorphism $\mathcal{J}_{L} \longrightarrow \mathcal{J}_{L}^{\wedge}$ with the desired property. Since $E$ is defined over $K$, this isomorphism descends to

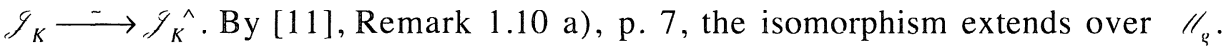
$\mathrm{O}$ 
Corollary. There exists a line bundle on $\mathscr{J}$ whose relative first Chern class (with respect to $\varphi$ ) is $2 E$.

Proof. Pull-back the Poincaré bundle on $\mathscr{J} \times_{\|_{p}} \mathscr{J}^{\wedge}$ via $(1, \mathscr{A})$ :

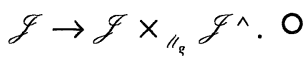

\$5.5. Proposition. The first Chern class induces a surjection $c_{1}: \operatorname{Pic}\left(\mathscr{J}^{d}\right) \rightarrow$ $H_{a n}^{2}(\mathscr{J}, \mathbb{Z})$ and we have an exact sequence:

$$
0 \rightarrow \operatorname{Pic}\left(\mathscr{M}_{g}\right) \otimes_{\mathbf{Z}} \mathbb{Q} \rightarrow c_{1}\left(\operatorname{Pic}\left(\mathscr{J}^{d}\right)\right) \otimes_{\mathbf{Z}} \mathbb{Q} \rightarrow \mathbb{Q} E \rightarrow 0
$$

where the injection is the pull-back via $\mathscr{J}^{d} \rightarrow \mathbb{M}_{g}$ and the surjection is the relative first Chern class with respect $\varphi$.

Proof. Clear from the preceding paragraphs. At first it may appear that $c_{1}$ is only surjective up to torsion, but it follows from the Kummer and exponential exact sequences that the cokernel of $c_{1}$ is always torsion-free. $O$

\$5.6. Let $\mathscr{C}^{-d}=\mathscr{E} \times{ }_{1,} \mathscr{J}^{d}$. Write $\psi_{d}: \mathscr{C}^{-d} \rightarrow \mathscr{J}^{d}$. Denote by $\kappa_{\alpha} \in H^{2},\left(\mathscr{J}^{d}\right.$, $\mathbf{G}_{m}$ ) the obstruction to the existence of a universal bundle of degree $d$ on $\mathscr{E}^{\sim d}$. Write $i_{d}: \mathscr{J}^{d} \rightarrow \mathscr{P}$ for the natural inclusion. From the Leray spectral sequence of $\psi_{d}$ for the étale sheaf $\mathbb{G}_{m}$, we get an exact sequence:

$$
\begin{gathered}
\operatorname{Pic}\left(\mathscr{J}^{d}\right) \\
\downarrow_{\text {natural Identfication }} \\
H^{1}{ }_{t}\left(\mathscr{G}^{\sim, d}, \mathbb{G}_{m}\right) \longrightarrow H_{t}^{0}\left(\mathscr{J}^{d}, \mathbb{R}^{1}{ }_{t} \psi_{d,} \mathbb{G}_{m}\right) \stackrel{\gamma}{\longrightarrow} H_{t}{ }_{t}\left(\mathcal{J}^{d}, \mathbb{G}_{m}\right)
\end{gathered}
$$

By general nonsense, $\kappa_{d}=\gamma(\mathrm{id})$. From the existence of $\omega$ on $\mathscr{E}$, one sees that under the identification $\mathscr{\epsilon}^{-d} \rightarrow \mathscr{\epsilon}^{-d+\chi}$ (via $\omega$ ), we have $\kappa_{d}=\kappa_{d+\chi}$. On the other hand, if $d>\chi$, then the functor $\operatorname{Div}^{d}$ of effective divisors of degree $d$ is a Brauer-Severi stack over $\mathscr{J}^{d}$ (by which we mean that it is a projective bundle in the étale topology), whose associated class in $H_{t}^{2}\left(\mathscr{J}^{d}, \mathbb{G}_{m}\right)$ is clearly $\kappa_{d}$. Thus we see that for all $d, \kappa_{d}$ is a torsion element.

§5.7. Proposition. $c_{1}: \operatorname{Pic}\left(\mathscr{J}^{d}\right) \rightarrow H_{a n}^{2}\left(\mathscr{J}^{d}, \mathbb{Z}\right)$ is injective.

Proof. If suffices to prove that for $d$ large, $\operatorname{Pic}\left(\mathscr{J}^{d}\right)$ is finitely generated (since the kernel of $c_{1}$ must be infinitely divisible). Now if $\mathscr{X}=\mathscr{C} \times{ }_{1 "} \times \ldots \times{ }_{10} \mathscr{C}$ (the $d$-fold product), then we know that Pic $(\mathscr{X})$ is finitely generated. Now we 
have a natural finite flat map $\mathscr{X} \rightarrow \operatorname{Div}^{d}$; hence, via the associated norm map $\operatorname{Pic}(\mathscr{X}) \rightarrow \operatorname{Pic}\left(\operatorname{Div}^{d}\right)$, we see that $\operatorname{Pic}\left(\operatorname{Div}^{d}\right)$ is finitely generated. We also have a natural map $\pi: \operatorname{Div}^{d} \rightarrow \mathscr{J}^{d}$ (which is a Brauer-Severi morphism), so if $\mathscr{L}$ is a line bundle on $\mathscr{J}^{d}$, then $\mathscr{L} \cong \pi_{1} \pi^{\prime} \mathscr{L}$. Thus $\pi^{\prime}: \operatorname{Pic}\left(\mathscr{J}^{d}\right) \rightarrow \operatorname{Pic}\left(\operatorname{Div}^{d}\right)$ is an injection, and we are done. $\bigcirc$

§5.8. We now go back to working over an arbitrary base, which we denote by a subscript. Let $X=\mathscr{C}$ or $\mathscr{J}^{d}$. Then we have the following

Proposition. The natural map $\operatorname{Pic}\left(X_{\mathrm{Z}}\right) \rightarrow \operatorname{Pic}\left(X_{\mathrm{C}}\right)$ is injective with finite cokernel.

Proof. Injectivity follows from the fact that $H^{0}\left(X_{R}, c_{X_{R}}\right)=R$ for any ring $R$. The rest follows from the preceding paragraph. 0

§5.9. Given any relative Brauer-Severi stack $f: X \rightarrow S$, we obtain a bundle of Grassmannians $f^{\sim}: X^{\sim} \rightarrow S$ (in the étale topology) by considering the lines in the projective spaces of the fibres of $f$. Applying this construction to $\operatorname{Div}^{d} \rightarrow \mathscr{J}^{d}$ (for $d>\chi)$, we obtain a map $\psi: \mathscr{G}^{d} \rightarrow \mathscr{J}^{d}$. Denote by $\zeta$ the canonical bundle on $\mathscr{G}^{d}$ for $\psi$. Write $\operatorname{Pic}_{\mathbf{Q}}\left(\right.$ ?) for $\operatorname{Pic}\left(\right.$ ?) $\otimes_{\mathbf{Z}} \mathbf{Q}$. We call elements of $\operatorname{Pic}_{\mathbf{Q}}($ ?) "virtual line bundles". Denote by $\gamma \in \mathrm{Pic}_{\mathrm{Q}}\left(\mathscr{G}^{d}\right)$ the $\mathbb{Q}$-multiple $\zeta$ such that restricted to the fibres of $\psi$, it is the line bundle that defines the Plücker embedding.

Definition. We shall refer to the following diagram as the main fibration:

$$
\mathscr{G}^{d} \stackrel{\psi}{\longrightarrow} \mathscr{J}^{d} \stackrel{\varphi}{\longrightarrow} \mathbb{N}_{g}
$$

We now wish to define a canonical element $\Theta^{d} \in \operatorname{Pic}_{\mathbf{Q}}\left(\mathscr{J}^{d}\right)$ which has relative first Chern class with respect to $\varphi$ equal to $E$. We do this as follows. Since $g^{0} \rightarrow \mathscr{M}_{\mathrm{g}}$ has the canonical identity section, we can define $\Theta^{0}$ by requiring that the pull-back via the identity section be trivial. For arbitrary $d$, we consider $\mu_{d}: \mathscr{J}^{d} \rightarrow \mathscr{J}^{0}$ given by composing the map "multiplication by $\chi$ " from $\mathscr{J}^{d}$ to $g^{d \chi}$ with the map "subtract off $\omega^{\otimes d}$ " from $g^{d \chi}$ to $g^{0}$. Then we define $\Theta^{d}$ to be $\left(\frac{1}{\chi^{2}}\right) \mu_{d}^{\prime} \Theta^{0}$. Note that if $\chi$ divides $d$, with $\chi=N d$, then $\Theta^{d}=$ the pull-back of $\Theta^{0}$ via the map "subtract off $\omega^{\otimes N}$ " from $g^{d}$ to $g^{0}$. When $d$ is fixed, we shall often omit it in the symbol $\Theta^{d}$.

$\$ 5.10$. Theorem. $\operatorname{Pic}\left(X_{R}\right)$ is finitely generated, for $X=\mathscr{l}_{g} ; \mathscr{J}^{d} ;$ or $\xi^{d}$ and $R$ $=\mathbb{Z}$ or $\mathbb{C}$. Moreover, for such $R$, 


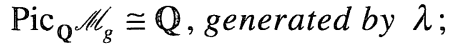

$\operatorname{Pic}_{\mathrm{Q}^{J^{d}}} \cong \mathbb{Q}^{2}$, generated by $\varphi \lambda$ and $\Theta$;

$\operatorname{Pic}_{Q^{G}} \mathscr{G}^{d} \cong \mathbb{Q}^{3}$, generated by $\gamma,(\varphi \psi)^{r} \lambda$, and $\psi * \Theta$.

(For convenience, we shall write $\lambda$ instead of $\varphi^{\prime} \lambda$, etc.) Thus via pull-back, we obtain a filtration, which we shall refer to as the main filtration, on $\operatorname{Pic}_{Q} \mathscr{G}^{d}$ which has subquotients of dimension 1: $0=F^{0} \subseteq F^{1} \subseteq F^{2} \subseteq F^{3}=\mathrm{Pic}_{\mathrm{Q}} \mathscr{G}^{d}$, where $F^{1} \cong \operatorname{Pic}_{\mathbf{Q}} \mathbb{M}_{g}$, and $F^{2} \cong \operatorname{Pic}_{\mathbf{Q}} \mathcal{J}^{d}$.

Proof. Clear from the previous results in this Section. 0

\section{§6. The Main Fibration}

§6.1. In this Section, we study the "the main fibration" defined in $\S 5$. We construct various maps from Hurwitz-type schemes into $\mathscr{G}$, and use these to prove some facts about the Picard group of the Hurwitz scheme and of $\mathscr{G}$. (Here $d$ is fixed, and so we write $\mathscr{G}$ for $\mathscr{G}^{d}$.)

\section{\$6. The Excess Divisors in the Main Fibration}

$\S 6.2$. We return to working over the ring $R$ of $\S 1$. We fix $b, d$, and $g$, and assume further that $g \geq 5$, and that $d>2 g+4$. For simplicity we shall denote $\mathscr{H}_{\mathscr{L}} \mathcal{F}_{b . d}$ by $\mathscr{H}$ and. $\mathscr{I}_{b, d}$ by $\overline{\mathscr{H}}$. Let us denote by $\mathscr{H}^{\sim}$ the open substack of $\overline{\mathscr{H}}$ that parametrizes admissible coverings with the following two properties:

(1) the nodal curve upstairs has an irreducible component which is smooth of genus $g$;

(2) the genus zero curve downstairs has at most two irreducible components.

Let us note that under the natural map $\overline{\mathscr{H}} \rightarrow \overline{\mathbb{I}}_{g}$ (\$3.27), $\mathscr{H}^{-}$maps into $\mathscr{M}_{g}$. On the other hand, if we denote $l \varkappa_{b . d}$ by $\overline{\mathscr{H}}^{+}$, then we have an étale covering $f: \overline{\mathscr{H}}^{+} \rightarrow \overline{\mathscr{H}^{\prime}}$. Thus $f$ is a Galois covering with Galois group equal to $\Gamma \stackrel{\text { def }}{=} \mathscr{f}^{b}$. We denote by $\mathscr{H}^{+}, \mathscr{H}^{\prime++}$ the pull-backs of $\mathscr{H}$, $\mathscr{H}^{\prime}$ via $f$. Thus we have a commutative diagram:

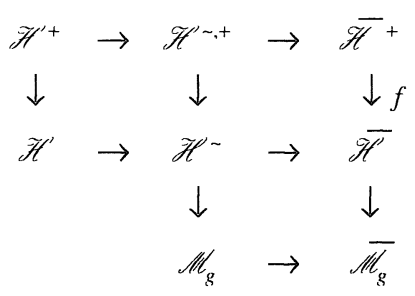


where all the horizontal arrows are open immersions.

§6.3. Let us note that the relative dimension of $\mathscr{G}$ over $R$ is: $2((d+1-g)-2)+g+3(g-1)=2 d+2 g-2-3=b-3$ (by the Riemann-Hurwitz formula). But this is the dimension (over $R$ ) of $\mathscr{H}$. In fact, we have the following

Proposition. There is a natural open immersion $\kappa: \mathscr{H}^{\prime} \rightarrow \mathscr{G}$.

Proof. Let $S$ be an $R$-scheme. Then elements $\mathscr{H}^{+}(S)$ correspond to diagrams:

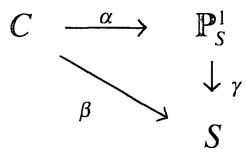

where $C \rightarrow S$ is a smooth curve of genus $g$, and $C \rightarrow \mathbb{P}_{S}^{1}$ is a degree $d$ and has only simple ramification. By assigning to this element the triple $\left\{(C \rightarrow S) \in \mathbb{M}_{g}(S)\right.$, $\alpha^{\prime}(\overbrace{\mathbf{P}_{S}^{\prime}}(1)) \in \mathscr{J}^{d}(S),\left(\gamma_{1}\left(\left(_{\mathbf{P}_{S}^{\prime}}(1)\right) \rightarrow \beta_{1}\left(\alpha^{\prime}(\overbrace{\mathbf{P}_{S}^{\prime}}(1))\right)\right) \in \mathscr{G}(S)\right\}$, we obtain a morphism $\mathscr{H}^{+} \rightarrow \mathscr{G}$ which is clearly invariant under the action of $\Gamma$. Hence we get a morphism $\kappa: \mathscr{H} \rightarrow \mathscr{G}$. It is clear from the definitions and by looking at the situation when $S$ is the spectrum of an artinian ring that $\kappa$ is étale. It is also clear from the definitions that $\kappa$ is injective on geometric points. Thus $\kappa$ must be an open immersion. $O$

\$6.4. The next order of business is to understand what the complement $\xi \rightarrow \kappa\left(\mathscr{H}^{\prime}\right)$ looks like. To do this, we work over an algebraically closed field $k$ (that is an $R$-algebra). Let $C$ be a smooth, proper, connected curve over $k$, and let $\mathscr{Z}$ be a line bundle of degree $d$ on $C$. Let $V=\Gamma(C, \mathscr{L}), P=\mathbb{P}_{h}\left(V^{\wedge}\right)$, where "^" for us will always mean the dual space. Thus $\operatorname{dim}_{h} V=d+1-g, \operatorname{dim}_{h} P=d-g$, and $P$ may be thought of as the space of effective divisors that give rise to $\mathcal{Z}$. A map $\alpha: C \rightarrow \mathbb{P}_{h}^{1}$ (considered up to automorphisms of $\left.\mathbb{P}_{h}^{1}\right)$ such that $\alpha^{\prime}\left(/_{\mathbf{P}_{l}^{\prime}}(1)\right)=\mathscr{L}^{\prime}$ may be thought of as a line $L$ in $P$ such that:

(1) There doesn't exist any point $p \in C(k)$ that appears in every divisor in $L$.

On the other hand, what does it mean to say that $\alpha$ has at most simple ramification? It means that every divisor in $L$ that is not made up of $d$ distinct points has $d-1$ distinct points, so only one point has coefficient not equal to 1 , and that coefficient is 2 . In other words, we must require that: 
(2) $L$ doesn't include a divisor that contains $3 p$ for some $p \in C(k)$;

(3) $L$ doesn't include a divisor that contains $2 p+2 q$ for some $p, q \in C(k)$.

Now let $G=$ the Grassmannian of lines in $P$. Then $\operatorname{dim}_{k} G=2(d-g-1)$. Let $\delta_{G}$ denote the ample generator of the Picard group of $G$. If we think of $G$ as parametrizing rank 2 quotients $V^{\wedge} \rightarrow \mathscr{Q}$, then $\delta_{G}$ corresponds to $\wedge^{2} \mathscr{Q}$. Let us consider the diagram:

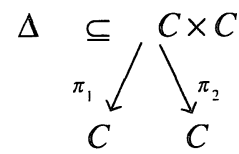

(Diagram 6.1)

Let $i$ be such that $d-i>2 g-2$. We consider the line bundles $\mathscr{M}^{(t)}=\left(\pi_{1}^{\prime} \mathscr{L}\right)(-i \Delta)$. Let $\mathscr{E}^{\prime}=\pi_{2,1}\left(\mathscr{M}^{(t)}\right)$; it has rank $d-g+1-i$. Let us denote by $\mathbf{G}$ (a vector bundle) the Grassmannian of 2-dimensional quotients of the dual of the vector bundle. Now clearly we have a natural locally split injection $\mathscr{E}^{1} \rightarrow \mathscr{E}^{0}$ which gives rise to a morphism $\rho_{3}: \mathbb{G}\left(\mathscr{E}^{1}\right) \rightarrow \mathbb{G}\left(\mathscr{E}^{0}\right)$. Now $\mathscr{E}^{0}$ is simply the pull-back to $C$ of the $k$ vector space $V$, so $\mathbb{G}\left(\mathscr{E}^{0}\right)=C \times G$. If we compose $\rho_{3}$ with the projection to $G$, we obtain a morphism $\sigma_{3}: \mathbb{G}\left(\mathscr{E}^{1}\right) \rightarrow G$ which is clearly generically injective and whose image consists precisely of the lines $L$ that do no satisfy (1). Since $\operatorname{dim}_{k} \mathbb{G}\left(\mathscr{E}^{1}\right)=\operatorname{dim}_{k}(G)-1$, this image is some irreducible effective divisor $D_{3}$, whose degree we will compute in $§ 6 \mathrm{~B}$.

We also have a natural locally split injection $\mathscr{E}^{3} \rightarrow \mathscr{E}^{0}$ which gives rise to a morphism $\mathbb{P}\left(\mathscr{E}^{3, \wedge}\right) \rightarrow \mathbb{P}\left(\mathscr{E}^{0, \wedge}\right)=C \times P$. If we then compose with the projection to $P$, we get a morphism $\rho_{1}: \mathbb{P}\left(\mathscr{E}^{3, \wedge}\right) \rightarrow P$ which is clearly generically injective and whose image consists exactly of the bad divisors that appear in (2). The image in $P$ is an irreducible variety $V_{1}$ of codimension 2 . Let us now consider the incidence variety $I \subseteq P \times G$ of points $(p, L)$ with $p \in P(k), L \in G(k)$ such that the point $p$ lies on the line $L$. Then it is well-known that $I$ is a $\mathbb{P}^{1}$-bundle over $G$ and a $\mathbb{P}^{(d-g-1)}$-bundle over $P$. Denote by $I_{V_{1}}$ the pull-back of $I$ (regarded as an object over $P$ ) via $V_{1} \rightarrow P$. Let $\sigma_{1}: I_{V_{1}} \rightarrow G$ denote the composition of the natural inclusions $I_{V_{1}} \subseteq I \subseteq P \times G$ with the projection to $G$. It is clear (by elementary algebraic geometry) that $\sigma_{1}$ is generically injective. On the other hand, $\operatorname{dim}_{h} I_{V_{1}}=\operatorname{dim}_{h}(G)-1$, so the image of $I_{V_{1}}$ in $G$ is some irreducible divisor $D_{1}$ whose points are exactly the bad lines that appear in condition (2). We shall compute the degree of $D_{1}$ in $\S 6 \mathrm{~B}$. 


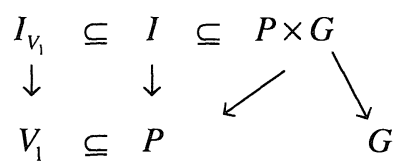

Now let us consider the following diagram:

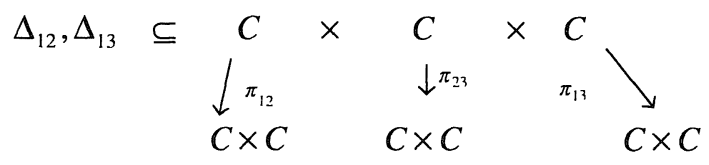

(Diagram 6.2)

where the $\pi_{l j}$ 's are the respective projections and $\Delta_{l j}=\pi_{l j}^{-1}(\Delta)$. Let us suppose that $i, j$ are such that $d-i-j>2 g-2$. Let $\mathscr{F}^{(1, j)}=\left(\pi_{1}^{\prime} \mathscr{L}\right)\left(-i \Delta_{12}-j \Delta_{13}\right)$. Let $\mathscr{K}^{(1, j)}=\pi_{23,1} \mathscr{F}^{(1, j)}$. Then $\mathscr{K}^{(1, j)}$ is a vector bundle on $C \times C$ of rank $d+1-g-i$ $-j$. Note that we have a natural locally split injection $\mathscr{K}^{(2.2)} \rightarrow \mathscr{K}^{(0.0)}$ which gives rise to a morphism $\mathbf{P}\left(\mathscr{K}^{(2.2) \wedge}\right) \rightarrow \mathbf{P}\left(\mathscr{K}^{(0,0) \wedge}\right)=C \times C \times P$. Composing with the projection $P$, we get a morphism $\mathbf{P}\left(\mathscr{K}^{(2,2) \wedge}\right) \rightarrow P$ which is clearly generically injective after taking the quotient by the action of $\mathbb{Z} / 2 \mathbf{Z}$ permuting the two factors of $C$, and whose image consists exactly of the bad divisors that appear in (3). The image is an irreducible variety $V_{2}$ of codimension 2 in $P$. Forming $I_{V_{2}}$ and $\sigma_{2}: I_{V_{2}} \rightarrow G$ as before, we see that $\sigma_{2}$ is generically injective, and that its image in $G$ is some irreducible divisor $D_{2}$ whose points are exactly the bad lines that appear in condition (3). We shall compute the degree of $D_{2}$ in $\S 6 \mathrm{~B}$.

In particular, since $G \neq D_{1} \cup D_{2} \cup D_{3}$, we see that we can always construct a map $\alpha: C \rightarrow \mathbf{P}^{1}$ with at most simple ramification. Thus $\psi \circ \kappa$ is surjective. Note that instead of working over $k$, then, we could have worked over $\mathscr{J}^{d}$. In summary, we have the following:

Proposition. The complement of $\kappa(\mathscr{H})$ in $\mathscr{G}$ consists of three relative

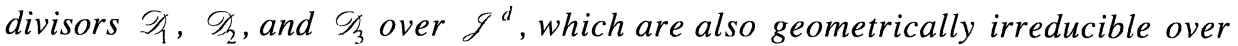
$\mathcal{J}^{d}$. They correspond, respectively, to the linear pencils that fail to meet one of the conditions (2), (3), or (1) listed at the beginning of this $\S$.

$\S 6.5$. We now wish to relate these divisors of $\mathscr{G}$ to the divisors of $\overline{\mathscr{H}}$ constructed in $\S 4$. To do this, we shall need to construct some sort of map from $\overline{\mathscr{H}}$ (or at least part of it) into $\mathscr{G}$. This is a somewhat delicate task, which we now undertake.

We shall call divisors at infinity of $\mathscr{H}^{\text {-.+ }}$ or $\overline{\mathscr{H}}^{+}$that lie over divisors of species $X$ in $\mathscr{H}^{\sim}$ or $\overline{\mathscr{H}}$ "divisors of species $X$ ", as well. We propose to extend 
the map $\mu^{\prime}: \mathscr{H}^{+} \longrightarrow \mathscr{H} \stackrel{\kappa}{\longrightarrow} \mathscr{G}$ to a map $\mu: \mathscr{H}^{\prime++} \rightarrow \mathscr{G}$. For any two distinct integers $i$ and $j$ between 1 and $b$, let us denote by $\mathscr{U}_{1 j}$ the open substack of $\mathscr{H}^{\text {-. }}$ parametrizing admissible covers for which the marking sections numbered $i$ and $j$ of the curve downstairs lie in the irreducible component of the curve downstairs which is the image of the curve of genus $g$ upstairs. Then it is easy to see that, as $i, j$ ranges over all admissible values, the union of the $\mathscr{Z}_{l J}$ is all of $\mathscr{H}^{\text {r.t}}$, while the intersection of the $\mathscr{H}_{l J}$ is $\mathscr{H}^{+}$. Thus, to define our extension $\mu$, it suffices to define, on each $\mathscr{U}_{l \jmath}$, a morphism $\mathscr{H}_{l \jmath} \rightarrow \mathscr{G}$ that restricts to $\mu^{\prime}$ on $\mathscr{H}^{+}$. Let $f: \mathscr{C} \rightarrow \mathscr{P}$ denote the universal admissible covering over $\mathscr{H}^{\boldsymbol{N}^{+}}$. Let $\sigma_{1}, \ldots, \sigma_{b}: \mathscr{H}^{\sim+} \rightarrow \mathscr{P}$ denote the marking sections. Let $E_{l}$ denote the schematic inverse image via $f$ of $\sigma_{1}$. Let $\mathscr{C}^{\prime} \rightarrow \mathscr{H}^{\text {,++}}$ denote the smooth curve of genus $g$ obtained by blowing down $\mathscr{C}$ as in the diagram of $\$ 3.27$; thus we have a blowdown map $\pi: \mathscr{E} \rightarrow \mathscr{E}^{\prime}$. Let $E_{t}^{\prime}$ denote the push-forward of the divisor $E_{l}$ via $\pi$. Since $\mathscr{C}^{\prime}$ is a regular scheme, $E_{l}^{\prime}$ defines a line bundle $\mathscr{L}_{1}$ on $\mathscr{C}^{\prime}$, along with a global section $s_{t}$ of $\mathscr{L}_{1}$ corresponding to $E_{1}^{\prime}$. Now $\mathscr{L}_{1}$ defines a morphism $v_{l}: \mathscr{H}^{\sim++} \rightarrow \mathscr{J}^{d}$ (the universal Jacobian). Since it is clear that $v_{\imath}$ and $v_{J}$ coincide when restricted to $\mathscr{H}^{+}$, it follows (since $\mathscr{H}^{-+}$is separated and integral) that $v_{1}=v_{j}$. Now it follows from the definition of $\mathscr{Z}_{t j}$ that over $\mathbb{Z}_{t J}$, the restrictions of $E_{l}^{\prime}$ and $E_{\jmath}^{\prime}$ to any geometric fibre of $\mathscr{C}^{\prime} \rightarrow \mathscr{H}^{\prime++}$ are distinct divisors. Thus, over $\mathscr{Z}_{l \jmath}$, the pair $E_{l}^{\prime}$ and $E_{\jmath}^{\prime}$ define a pencil, that is, a morphism $\mu_{\imath \jmath}: \mathscr{t}_{l \jmath} \rightarrow \mathscr{G}$ whose composite with the projection $\mathscr{G} \rightarrow \mathscr{J}^{d}$ is $v_{1}=v_{j}$. Clearly this morphism restricts to $\mu^{\prime}$ on $\mathscr{H}^{+}$. Thus, gluing together the $\mu_{t \prime}$, we obtain our $\mu$. The behavior of $\mu$ on divisors at infinity is given by the following

Proposition. Suppose that $d>2 g+4$. Then $\mu$ maps divisors of Species 1 (resp. 2, 3B*) into $\mathscr{Z}_{1}$ (resp. $\mathscr{Z}_{2}, \mathscr{Z}_{3}$ ); moreover, the restriction of $\mu$ to the excess divisors is a quasi-finite morphism. On the other hand, $\mu$ maps the nonexcess divisors to subsets of codimension at least two that lie outside the image of $K$. In particular, the inverse image divisor via $\mu$ of $\mathscr{Z}_{1}$ (resp. $\mathscr{Z}_{2}, \mathscr{Z}_{3}$ ) is a positive linear combination of all the divisors of $\mathscr{H}^{\prime n^{+}}$of Species 1 (resp. 2, $\left.3 B^{*}\right)$. Finally, the image via $\mu$ of any excess divisor is dense in the restriction of the corresponding $\mathscr{Z}_{1}$ to any geometric fibre of $\mathscr{G} \rightarrow \mathbb{M}_{\mathrm{g}}$.

Proof. First, we note that it follows immediately from the definition of $\mu$ and the explicit descriptions of the excess divisors given in $\S 4$ that $\mu$ maps a divisor of Species 1 (resp. 2, 3B*) into $\mathscr{Z} /($ resp. $\mathscr{Z} / 2, \mathscr{Z} / 3$ ); the assertion on quasifiniteness is also clear from the explicit descriptions in $\S 4$.

Now let us prove the final statement. We fix a geometric point $c: \operatorname{Spec}(k) \rightarrow \mathscr{M}_{g}$, where $k$ is an algebraically closed field (of characteristic 
greater than $b$ ). Then it suffices to show that the generic point of $\left.\mathscr{L}_{1}\right|_{c}$ (for $i=1$, 2,3 ) corresponds to a morphism $C \rightarrow \mathbb{P}^{1}$ which appears in an admissible covering of Species 1,2 , or $3 B^{*}$. Let us first note that the three irreducible divisors $\mathscr{D}, I_{c}$ in $G(i=1,2,3)$ are distinct: there are many ways to see this; one way is simply to note that if any two were equal, then their degrees, as computed in Theorem 21 of $\S 6 \mathrm{~B}$ would coincide; but an elementary calculation reveals that this is absurd. We now proceed case by case.

For $i=1$, a simple combinatorial check reveals that it suffices to show that the generic pencil in $\left.\mathscr{D}_{1}\right|_{c}$ does not contain two divisors each of which has a point of multiplicity three. But in the notation of $\$ 6.4$, this amounts to checking that the set of lines in $P$ that pass through two points of $V_{1}$ has codimension at least two in $G$, but this is clear.

For $i=2$, there are two points to check: first, that the generic pencil does not contain two divisors each of which has two points of multiplicity two; but this follows by the same argument as in the previous paragraph. Second, we must check that the generic pencil does not contain a divisor which has three points of multiplicity two; but the subvariety $V_{3} \subseteq P$ consisting of divisors with three points of multiplicity two has codimension three in $P$ (since we assume that $d>2 g+4$ ). Thus, the subvariety of $G$ consisting of lines in $P$ that pass through $V_{3}$ has codimension two, as desired.

For $i=3$, here we need to show that the generic pencil does not contain two base points. But one sees immediately that the subvariety of $G$ corresponding to pencils with two base points has dimension $2(d-3)+2=2 d-4$, i.e., has codimension two in $G$, as desired.

We now turn to considering the nonexcess divisors. Let us suppose that $x: \operatorname{Spec}(k) \rightarrow \mathscr{H}^{-+}$is a geometric point that lands in a nonexcess divisor. One piece of information of an admissible covering that is clearly preserved by $\mu$ is the unique nonconstant morphism, which we shall henceforth refer as the central morphism of the admissible covering, from the curve of genus $g$ upstairs to a copy of $\mathbb{P}^{1}$ downstairs. Thus, let us suppose that $\mu(x)$ lands inside the image of $\kappa$. Then the central morphism of $x$ must be ramified over $b$ distinct points downstairs; but since the curve downstairs in the admissible covering $x$ is stable, this could only happen if the curve downstairs in $x$ is, in fact, smooth, which contradicts the assumption that $x$ lies in a nonexcess divisor. Thus, $\mu(x)$ must land inside one of the $Z_{1}$. Now let us suppose that $\mu(x)$ lands in a sufficiently generic point of $\mathscr{O} /$. Then by our proof of the final statement of the Proposition, it follows that the central morphism of $x$ is ramified over exactly $b-1$ distinct points downstairs; thus, since the curve downstairs in the admissible covering $x$ is stable, 
it follows by the same reasoning as before, that the curve downstairs in $x$ must be of type $(b-2,2)$. On the other hand, we gave explicit descriptions in $\$ 4$ of all the divisors of type $(b-2,2)$; going down the list, one sees that the only possibility now for the nonexcess divisor in which $x$ sits is Species $3 B$ with $g_{1}=g, g_{2}=0$. But if $d_{2}>1$, then the central morphism of $x$ must have at least two base points, which contradicts what we just saw in the previous paragraph. 0

$\S 6.6$. At this point, we need to employ a result which will not be proven until the end of $\S 7$. We state it here for ease of reference:

Theorem. Under the assumption that $d \geq 2 g+4$, the line bundles defined by $\mathscr{Z}_{1}, \mathscr{Z}_{2}$, and $\mathscr{D}_{3}$ are linearly independent in the Picard group of $\mathscr{G}$.

§6.7. Now by elementary algebraic geometry, we have an exact sequence:

$$
\mathbb{Z} \mathscr{Q}_{1} \oplus \mathbb{Z} \mathscr{Z}_{2} \oplus \mathbb{Z} \mathscr{Z}_{3} \rightarrow \operatorname{Pic} \mathscr{G} \rightarrow \operatorname{Pic} \mathscr{H} \rightarrow 0
$$

If we tensor with $\mathbb{Q}$, and use the fact $(\S 5)$ that $\operatorname{Pic}_{\mathbf{Q}} \mathscr{G}=\mathbb{Q}^{3}$, then we obtain the following (again under the assumption that $d>2 g+4$ )

Corollary. $\mathscr{\mathscr { Z } _ { 1 }}, \mathscr{Z}_{2}$, and $\mathscr{Z} / 3$ form a basis of $\mathrm{Pic}_{\mathrm{Q}} \mathscr{E}$.

Corollary. $\operatorname{Pic}(\mathscr{H})$ is a finite group. The homomorphism $\sum$ of $\$ 4.8$, is a surjection when tensored with $\mathbb{Q}$.

Remark. Suppose we knew a priori or through some other technique that $\operatorname{Pic}(\mathscr{H})$ is finite. Then what we have done here would prove that $\operatorname{Pic}_{\mathbf{Q}} \mathscr{G}$ has dimension 3. This, in turn, would allow us to rederive in a more algebraic way many of the cohomological results of Harer (up to torsion) which were recalled in §5.3. Given all the explicit calculations done in the paper, it does not seem unreasonable to expect that one could, by closer examination, prove that $\operatorname{Pic}\left(\mathscr{H}^{\prime}\right)$ is finite is an a priori fashion. At the present time, however, the author has not succeeded in doing this.

§6.8. Remark. It is now of interest to compute the coefficient matrix relating the basis $\left\{\mathscr{Z}_{1}, \mathscr{Z}_{2}, \mathscr{Z}_{3}\right\}$ and the basis $\{\lambda, \Theta, \gamma\}$. We shall do this in the next Section.

\section{$\S 6 \mathbb{B}$ 。Intersection Theory Calculations}

\$6.9. Returning to the situation over an algebraically closed field $k$ as in the beginning of $\S 6.4$, the purpose of this Subsection is to compute the "degree" of the divisors $D_{1}, D_{2}$ and $D_{3}$ in $G$, where by "degree", we really mean the number $N$ 
such that a divisor is linearly equivalent to $\delta_{G}^{\otimes N}$. We begin by reviewing some basic facts about the geometry of Grassmannians.

$\S 6.10$. Lemma. Let $X \subseteq P$ be an irreducible variety of codimension 2 . Then if we construct $I_{X}$ and the associated generically injective morphism $I_{X} \rightarrow G$ as in $\S 6.4$, the degree of the image of this morphism in $G$ is equal to the degree of $X$ in $P$.

Proof. Let $e=$ the degree of $X$ in $P$. Then one knows (e.g. from [14]) that if one selects a linear subspace $Q \subseteq P$ of dimension 2 and a point $q \in Q$, the subvariety of $G$ formed of lines in $Q$ that pass through $q$ gives rise to a cycle which is dual to $\delta_{G}$. Thus it suffices to intersect $X$ with a generic linear subspace $Q \subseteq P$ of dimension 2 such that $X \cap Q$ has $e$ points $\left\{x_{1}, \ldots, x_{e}\right\}$, and to choose a generic $q \in Q$ that doesn't sit on any line passing through two of the $x_{l}$ 's. Then clearly there are exactly $e$ lines in $Q$ that pass through $q$ and intersect $X$, and moreover, the intersection between the image of $I_{X}$ in $G$ and the cycle associated to $\{Q, q\}$ is clearly transverse. Hence the Lemma. $O$

$\S 6.11$. Let $S$ be a smooth, proper, connected curve over $k$; let $\mathscr{E}$ be a vector bundle of rank $r+1$ on $S$. We use the Chow rings $A^{\prime}(-)$. Write $\varepsilon=c_{1}(\mathscr{E}) \in A^{1}(S)$. Let $P_{1}=\mathbb{P}(\mathscr{E}), \mathscr{L}_{1}=C_{P_{1}}(1)$. Then we have an exact sequence $0 \rightarrow \mathscr{F} \rightarrow \mathscr{C}_{P_{1}} \rightarrow \mathscr{L}_{1}$ $\rightarrow 0$, where the subscript denotes pull-back and $\mathscr{F}$ is defined by this exact sequence. Let $P_{2}=\mathbb{P}(\mathscr{F}), \mathscr{L}_{2}=\overbrace{P_{2}}(1)$. On the other hand, let $G^{\prime}=\mathbf{G}(\mathscr{E})$, and let $\mathscr{C}_{x}$ be the universal quotient of $\mathscr{E}$ on $G^{\prime}$. Let $P_{3}=\mathbb{P}(\mathscr{C}), \mathscr{L}_{3}=\mathscr{C}_{P_{3}}(1)$. Then clearly $P_{2} \cong P_{3}$, with $\mathscr{L}_{1}$ and $\mathscr{L}_{3}$ corresponding under this isomorphism. We thus have a diagram:

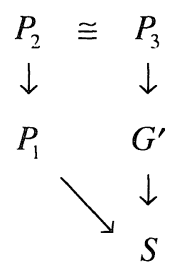

Let us denote $\wedge^{2} 6$ by $\delta^{\prime}$. Let $\kappa_{1}=c_{1}\left(Y_{1}\right) \in A^{\prime}\left(P_{1}\right)$ for $i=1,2,3$. Then we have

$$
\begin{aligned}
& A^{\prime}\left(P_{1}\right)=A(S)\left[\kappa_{1}\right] /\left(\kappa_{1}^{\prime+1}-\kappa_{1}^{\prime} \varepsilon\right), \text { so } \kappa_{1}^{N}=0 \text { for } N \geq r+2 . \\
& A^{\prime}\left(P_{3}\right)=A\left(G^{\prime}\right)\left[\kappa_{3}\right] /\left(\kappa_{3}^{2}-c_{1}(G) \kappa_{3}+c_{2}(\mathscr{C})\right) .
\end{aligned}
$$

Note that if we identify $A^{\prime}\left(P_{2}\right)$ and $A^{\prime}\left(P_{3}\right)$, we have $\kappa_{1}=\kappa_{3}, c_{1}(\mathscr{C})=\kappa_{1}+\kappa_{2}$, $c_{2}(\mathscr{G})=\kappa_{1} \kappa_{2}$. Let us denote the fundamental class of $S$ by $\eta$, so $\varepsilon=\operatorname{deg}(\mathscr{E}) \eta$. 
§6.12. Lemma. We have the following identities:

(1) $\kappa_{t}^{\prime+1}=\varepsilon \kappa_{,}^{\prime}$; hence $\kappa_{+}^{\prime+2}=0$ for $i=1,2$.

(2) $\kappa_{1}^{\prime-1} \kappa_{2}^{\prime}+\kappa_{1}^{r} \kappa_{2}^{\prime-1}=\varepsilon \kappa_{1}^{\prime-1} \kappa_{2}^{r-1}$;

(3) If $\varepsilon=0$, then $\kappa_{1}^{\prime-2} \kappa_{2}^{\prime}+\kappa_{1}^{\prime} \kappa_{2}^{\prime-2}=-\kappa_{1}^{\prime-1} \kappa_{2}^{\prime-1}$.

Proof. We give the proof assertion by assertion:

(1) This is elementary and well-known.

(2) $c_{t}(\mathscr{F})=(1-\varepsilon t)\left(1-\kappa_{1} t\right)^{-1}$. If we substitute formally by $t=\kappa_{2}^{-1}$ and multiply by $\kappa_{2}^{\prime}$, the result will be zero. So we do this and multiply further by $\kappa_{1}^{\prime-1}$ :

$0=\kappa_{2}^{\prime} \kappa_{1}^{\prime-1}\left(1-\frac{\varepsilon}{\kappa_{2}}\right)\left(1-\frac{\kappa_{1}}{\kappa_{2}}\right)^{-1}=\kappa_{2}^{\prime} \kappa_{1}^{\prime-1}+\kappa_{2}^{\prime-1} \kappa_{1}^{\prime}+\kappa_{2}^{\prime-2} \varepsilon \kappa_{1}^{\prime}-\varepsilon \kappa_{2}^{\prime-1} \kappa_{1}^{\prime-1}-\varepsilon \kappa_{2}^{\prime-2} \kappa_{1}^{\prime}$.

Rearranging terms gives the result.

(3) Same technique as in (2), but with $\varepsilon=0$, and we multiply by $\kappa_{1}^{\prime-2}$ instead

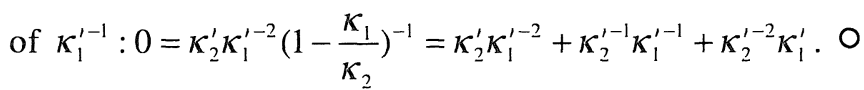

§6.13. Lemma。 $c_{1}\left(\delta^{\prime}\right)^{2,-1}=\left(\frac{2}{r+1}\right)\left(\begin{array}{c}2 r-1 \\ r\end{array}\right) \operatorname{deg}(\mathscr{E})$

Proof. Using the previous Lemma:

$$
\begin{aligned}
c_{1}\left(\delta^{\prime}\right)^{2,-1}= & \left(\kappa_{1}+\kappa_{2}\right)^{2,-1} \\
= & \varepsilon \kappa_{1}^{\prime-2} \kappa_{2}^{\prime}\left(\begin{array}{c}
2 r-1 \\
r+1
\end{array}\right)+\kappa_{1}^{\prime-1} \kappa_{2}^{\prime}\left(\begin{array}{c}
2 r-1 \\
r
\end{array}\right) \\
& +\kappa_{1}^{\prime} \kappa_{2}^{\prime-1}\left(\begin{array}{c}
2 r-1 \\
r
\end{array}\right)+\varepsilon \kappa_{1}^{\prime} \kappa_{2}^{\prime-2}\left(\begin{array}{c}
2 r-1 \\
r+1
\end{array}\right) \\
= & \varepsilon \kappa_{1}^{\prime-1} \kappa_{2}^{\prime-1}\left(\begin{array}{c}
2 r-1 \\
r
\end{array}\right)-\varepsilon \kappa_{1}^{\prime-1} \kappa_{2}^{\prime-1}\left(\begin{array}{c}
2 r-1 \\
r+1
\end{array}\right)
\end{aligned}
$$

Now the result follows from the fact that since $\kappa_{1}^{\prime} \kappa_{2}^{\prime-1} \eta$ is clearly the fundamental class of $P_{3}, \kappa_{1}^{\prime-1} \kappa_{2}^{\prime-1} \eta$ must be the fundamental class of $G^{\prime}$, and by the elementary 
calculation:

$\left(\begin{array}{c}2 r-1 \\ r\end{array}\right)-\left(\begin{array}{c}2 r-1 \\ r+1\end{array}\right)=\frac{(2 r-1) !}{r !(r-2) !}\left(\frac{1}{r-1}-\frac{1}{r+1}\right)=2 \frac{(2 r-1) !}{(r+1) !(r-1) !}=\left(\frac{2}{r+1}\right)\left(\begin{array}{c}2 r-1 \\ r\end{array}\right) .0$

§6.14. Lemman。 If $\varepsilon=0$, then $c_{1}\left(\delta^{\prime}\right)^{2(1-1)}=\frac{1}{r-1}\left(\begin{array}{c}2 r-2 \\ 2\end{array}\right)$.

Proof. Using §6.12:

$$
\begin{aligned}
c_{1}\left(\delta^{\prime}\right)^{2(\prime-1)} & =\left(\kappa_{1}+\kappa_{2}\right)^{2,-2} \\
& =\kappa_{1}^{\prime-2} \kappa_{2}^{\prime}\left(\begin{array}{c}
2 r-2 \\
r
\end{array}\right)+\kappa_{1}^{\prime-1} \kappa_{2}^{\prime-1}\left(\begin{array}{c}
2 r-2 \\
r-1
\end{array}\right)+\kappa_{1}^{\prime} \kappa_{2}^{\prime-2}\left(\begin{array}{c}
2 r-2 \\
r
\end{array}\right) \\
& =\kappa_{1}^{\prime-1} \kappa_{2}^{\prime-1}\left\{\left(\begin{array}{c}
2 r-2 \\
r-1
\end{array}\right)-\left(\begin{array}{c}
2 r-2 \\
r
\end{array}\right)\right\}
\end{aligned}
$$

$\operatorname{But}\left(\begin{array}{c}2 r-2 \\ r-1\end{array}\right)-\left(\begin{array}{c}2 r-2 \\ r\end{array}\right)=\frac{(2 r-2) !}{(r-1) !(r-2) !}\left(\frac{1}{r-1}-\frac{1}{r}\right)=\frac{(2 r-2) !}{r !(r-1) !}=\frac{1}{r-1}\left(\begin{array}{c}2 r-2 \\ r\end{array}\right) .0$

\$6.15. Propositiom. $\left[D_{2}\right]=d \delta_{G}$, where the brackets denote the linear equivalence class.

Proof. In the calculations of $\$ 6.11 \sim 14$, set $G^{\prime}=\mathbb{G}\left(\digamma^{1 \wedge}\right)$. Recall our map $\sigma_{2}: G^{\prime} \rightarrow G$. Clearly $\sigma_{3}\left(\delta_{G}\right)=\delta^{\prime}$ (i.e., the analogous bundle for $G^{\prime}$ to $\delta_{G}$ on $G$ ). Suppose $\left[D_{3}\right]=N \delta_{G}$. Since here $r+1=d-g$, we see from $\S 6.14$, that $c_{1}\left(\delta^{\prime}\right)^{2,-1}=N\left(c_{1}\left(\delta_{G}\right)\right)^{2 \prime}=N\left(\frac{1}{r}\right)\left(\begin{array}{c}2 r \\ r+1\end{array}\right)$. On the other hand, by $\S 6.13, c_{1}\left(\delta^{\prime}\right)^{2,-1}=$ $\left(\frac{2}{r+1}\right)\left(\begin{array}{c}2 r-1 \\ r\end{array}\right) \operatorname{deg}\left(\mho^{1, \wedge}\right)$. Thus $N=-\operatorname{deg}\left(\digamma^{1}\right)$. The result thus is a consequence of the following:

\$6.16. Lemmad. If $d-i>2 g-2$, then $\operatorname{deg}\left(\digamma^{\prime}\right)=-d i-(g-1) i(i-1)$.

Proof. Referring to Diagram 6.1 of $\$ 6.4$, we see that we have exact sequences:

$$
0 \rightarrow\left(\pi_{1} \searrow\right)(-(i+1) \Delta) \rightarrow\left(\pi_{1} \not\right)(-i \Delta) \rightarrow\left(\omega^{\otimes \imath} \otimes \not{\perp}\right)_{\Delta} \rightarrow 0
$$

Pushing forward by $\pi_{2}$, we get:

$$
0 \rightarrow \mathscr{F}^{1+1} \rightarrow \mathcal{E}^{1} \rightarrow \omega^{\otimes_{1}} \otimes \perp \rightarrow 0
$$


Hence we obtain $\operatorname{deg}\left(\mathscr{E}^{i+1}\right)=\operatorname{deg}\left(\mathscr{E}^{\prime}\right)-d-2 i(g-1)$. Since $\operatorname{deg}\left(\mathscr{E}^{0}\right)=0$, the result follows. $O$

§6.17. Proposition. $\left[D_{1}\right]=\{3 d+6(g-1)\} \delta_{G}=3(b-d) \delta_{G}$.

Proof. By $\S 6.10$, it suffices to compute the degree of $V_{1}$ in $P$ (notation as in the relevant part of $\S 6.4)$. Since $\rho_{1}^{*}\left(\mathscr{O}_{P}(1)\right)=\mathscr{O}_{\mathbf{P}_{(\times 3 \wedge)}}(1)$. Thus if $\alpha=c_{1}\left(\mathscr{O}_{P}(1)\right)$, $\alpha^{\prime}=c_{1}\left(\mathscr{O}_{\mathbf{P}\left(r^{2} \wedge\right)}(1)\right)$, and $V_{1}$ is of degree $N$, then $\left(\alpha^{\prime}\right)^{d-g-2}=N \alpha^{d-g}$. On the other hand, from basic facts about projective bundles, we know that $\left(\alpha^{\prime}\right)^{d-g-2}=$ $\operatorname{deg}\left(\mathscr{E}^{3, \wedge}\right)=-\operatorname{deg}\left(\mathscr{E}^{3}\right)$. Now apply $§ 6.16$.

§6.18. Lemma. Assuming, as usual, that $d>2 g+2$, we have the following Chern classes on $C \times C$ :

$$
\begin{aligned}
& c_{1}\left(\kappa^{(2,2)}\right)=-(b-4)\left(\eta_{1}+\eta_{2}\right)+4 \delta \\
& c_{2}\left(\kappa^{(2,2)}\right)=(b-4)(b-6)-24 g,
\end{aligned}
$$

where on $C \times C, \eta_{1}$ is the pull-back of the fundamental class of the $i^{\text {th }}$ factor, for $i$ $=1,2$, and $\delta=c_{1}(\Delta)-\eta_{1}-\eta_{2}$, where $\Delta$ is the diagonal.

Proof. First of all, $\mathscr{K}^{(0.2)}=\pi_{2} \mathscr{E}^{2}$. Thus $c\left(\mathscr{K}^{(0,2)}\right)=1-\{2 d+2(g-1)\} \eta_{2}$. On the other hand, we have exact sequences on $C \times C \times C$ :

$$
\begin{aligned}
& 0 \rightarrow \mathscr{F}^{(2,2)} \rightarrow \mathscr{F}^{(1,2)} \rightarrow \pi_{12}\left\{(\omega)_{\Delta}\right\} \otimes \pi_{13} \mathscr{M}^{(2)} \rightarrow 0 \\
& 0 \rightarrow \mathscr{F}^{(1,2)} \rightarrow \mathscr{F}^{(0,2)} \rightarrow \pi_{12}^{\prime}\left(C_{\Delta}\right) \otimes \pi_{13} \mathscr{M}^{(2)} \rightarrow 0
\end{aligned}
$$

Applying $\pi_{23,4}$, we obtain exact sequences on $C \times C$ :

$$
\begin{aligned}
& 0 \rightarrow \mathscr{K}^{(2.2)} \rightarrow \mathscr{K}^{(1.2)} \rightarrow \pi_{1}(\omega) \otimes \mathscr{M}^{(2)} \rightarrow 0 \\
& 0 \rightarrow \mathscr{K}^{(1.2)} \rightarrow \mathscr{K}^{(0.2)} \rightarrow \pi_{1}\left(\digamma_{C}\right) \otimes \mathscr{M}^{(2)} \rightarrow 0
\end{aligned}
$$

Now $c\left(\mathscr{M}^{(2)}\right)=1+(d-2) \eta_{1}-2 \eta_{2}-2 \delta$, where $\delta^{2}=-2 g, \delta \eta_{1}=\delta \eta_{2}=0$. Thus we obtain:

$$
\begin{aligned}
& c\left(\mathscr{K}^{(1,2)}\right)=c\left(\mathscr{K}^{(2,2)}\right)\left\{1+(d+2 g-4) \eta_{1}-2 \eta_{2}-2 \delta\right\} \\
& c\left(\mathscr{K}^{(0,2)}\right)=c\left(\mathscr{K}^{(1,2)}\right)\left\{1+(d-2) \eta_{1}-2 \eta_{2}-2 \delta\right\}
\end{aligned}
$$

Thus we have: 


$$
\begin{aligned}
c\left(\mathscr{K}^{(2,2)}\right)= & \left\{1-b \eta_{2}\right\}\left\{1+(d-2) \eta_{1}-2 \eta_{2}-2 \delta\right\}^{-1}\left\{1+(b-d-2) \eta_{1}-2 \eta_{2}-2 \delta\right\}^{-1} \\
= & \left\{1-b \eta_{2}\right\}\left\{1-(d-2) \eta_{2}+2 \eta_{2}+2 \delta-4(d-2) \eta_{1} \eta_{2}+4 \delta^{2}\right\} \\
& \left\{1-(b-d-2) \eta_{1}+2 \eta_{2}+2 \delta-4(b-d-2) \eta_{1} \eta_{2}+4 \delta^{2}\right\} \\
= & \left\{1-b \eta_{2}\right\}\left\{1-(b-4) \eta_{1}+4 \eta_{2}+4 \delta-6(b-4) \eta_{1} \eta_{2}+12 \delta^{2}\right\} \\
= & \left\{1-(b-4)\left(\eta_{1}+\eta_{2}\right)+4 \delta+(b-4)(b-6) \eta_{1} \eta_{2}+12 \delta^{2}\right\}
\end{aligned}
$$

○

$\S 6.19$. Let $S$ be a proper, smooth, connected surface over $k$. Let $\mathscr{E}$ be a vector bundle on $S$ of rank $r+1$. Let $\alpha=c_{1}\left(\mathscr{C}_{\mathbf{P}(r \wedge)}(1)\right)$. Then we have the following:

Lemma. $\alpha^{r+2}=c_{1}^{2}(\mathscr{E})-c_{2}(\mathscr{E})$.

Proof. We have $\alpha^{\prime+1}=-c_{1}(\mathscr{E}) \alpha^{\prime}-c_{2}(\mathscr{E}) \alpha^{\prime-1}$. Then $\alpha^{r+2}=-c_{1}(\mathscr{E}) \alpha^{\prime+1}$ $-c_{2}(\mathscr{E}) \alpha^{\prime}=\left\{c_{1}^{2}(\mathscr{E})-c_{2}(\mathscr{E})\right\} \alpha^{r}$, as desired. $\mathrm{O}$

$\S 6.20$. Proposition. $\left[D_{2}\right]=\frac{1}{2}\{b(b-10)+8 d\} \delta_{G}$.

Proof. As in $\$ 6.17$, we apply $\$ 6.10$, and we see that if we take $S=C \times C$ and $\mathscr{E}=\mathscr{K}^{(2,2)}$, so that $r+1=d-g-3$, we have $\frac{1}{2} \alpha^{\prime+2}=\left[D_{2}\right]$. Thus by $\S 6.19, \S 6.18$, we have $2\left[D_{2}\right]=2(b-4)^{2}-(b-4)(b-6)+(-32 g)+24 g=(b-4)(b-2)-8 g=b(b$ $-10)+8 d$. 0

$\S 6.21$. To summarize, we have the following

Theorem. The degrees of the excess divisors in $G$ are as follows:

$$
\begin{aligned}
& {\left[D_{1}\right]=3(b-d) \delta_{G}} \\
& {\left[D_{2}\right]=\frac{1}{2}\{b(b-10)+8 d\} \delta_{G}} \\
& {\left[D_{3}\right]=d \delta_{G}}
\end{aligned}
$$

\section{\$6C. Ramification Indices}

\$6.22. Let us consider the morphism $\mu: \mathscr{H}^{\sim+} \rightarrow$ constructed in $\$ 6.5$. Now $\mathscr{H}^{+} \subseteq \mathscr{H}^{\prime \sim+}$ as an open subscheme, and the complement of this open subscheme consists of certain divisors at infinity. In this Subsection, we wish to compute the indices of ramification of $\mu$ at the excess divisors. These computations will be important for $\S 7$. Note that we continue to assume here that $g \geq 5$ and $d>2 g+4$. 
Note that all this is taking place over $\mathbb{Z}\left[\frac{1}{N}\right]$, where $N=b$ !. On the other hand, $\mathscr{G}$ is defined over $\mathbb{Z}$, which prompts the question: What is the nature of the ramification of $\mu^{\prime}: \mathscr{H}^{+} \longrightarrow \mathscr{H} \stackrel{\kappa}{\longrightarrow} \mathscr{G}$, over the primes $p$ dividing $N$ ? In fact, we shall see that there is no ramification at the odd primes.

$\S 6.23$. We begin with the ramification of $\mu$ at the excess divisor of Species 2 . Note that in this case, the different (though not be discriminant) is étale over the base; thus, the process of "ordering the sections" gives rise to a covering of $\mathscr{G}$ which is étale over $\mathscr{Z} / 2$. Let $f: \mathscr{C} \rightarrow \mathscr{P}$ denote the universal admissible covering over $\mathscr{H}^{\text {-.+}}$. Let $\sigma_{1}, \ldots, \sigma_{b}: \mathscr{H}^{\text {n+t}} \rightarrow \mathscr{\mathscr { N }}$ be the $b$ marking sections; suppose that $\sigma_{\text {, }}$ lifts to $\rho_{1}: \mathscr{H}^{\sim,+} \rightarrow \mathscr{E}$, at which $f$ is simply ramified. Let $\wp$ be a prime divisor of height one of $\mathscr{H}^{\text {-+ }}$ which "is" a divisor at infinity of Species 2 . Let $U$ be an open substack of $\mathscr{H}^{-++}$that contains $\wp$ and is so small that the normal sheaves of the $\sigma_{b}$ (which are line bundles on $\mathscr{H}^{\prime++}$ ) are trivial when restricted to $U$. Choose trivializations $\tau_{1}, \ldots, \tau_{b}$ for these normal sheaves over $U$. Let $U^{\prime} \rightarrow U$ be the étale covering that parametrizes trivializations of the normal sheaves of the $\rho_{\imath}$ whose squares are the trivializations $\tau_{l}$. Let $\wp^{\prime}$ be a prime of $U^{\prime}$ that lies over $\wp$. Note that with this extra rigidification, a generic admissible covering of Species 2 loses all its automorphisms, so that $U^{\prime}$ is a scheme in a neighborhood of $\wp^{\prime}$. Let $A$ be the completion of $U^{\prime}$ at $\wp^{\prime}$. It is thus clear from the general theory of algebraic stacks and their associated coarse moduli spaces (see, e.g., [11], p. 23) that to determine the desired index of ramification. we must simply do the following: consider the group $\Gamma=\operatorname{Gal}\left(U^{\prime} / U\right) \cong(\mathbb{Z} / 2 \mathbb{Z})^{b}$ that acts on the added structure of choosing "specific square roots of uniformizers over points of simple ramification". The inertia subgroup $I$ at $\wp^{\prime}$ is isomorphic to $(\mathbb{Z} / 2 \mathbb{Z})$ and sits inside the $(\mathbb{Z} / 2 \mathbb{Z})^{2} \subseteq \Gamma$ that corresponds to the two points of simple ramification on the $\mathbb{P}^{1}$ 's upstairs. Then from the equivalent definition of $A$ as the solution to a certain infinitesimal moduli problem, we see that $I$ acts nontrivially on $A$. Let $B=A^{l}$, the ring of invariants. Then $B$ is also a discrete valuation ring, and the desired index of ramification is the same as that of $A$ over $B$. Looking at the explicit description given in [19], §4, p. 62, however, we see that $I$ takes some uniformizer of $A$ to minus itself: hence the index of ramification must be 2 .

Propositiom。 $\mu$ has ramification index 2 at excess divisors of Species 2.

$\$ 6.24$. Let us now note that $\mathscr{H}^{\prime-+}$ is already a scheme at the generic points of divisors of Species 1 and $3 B^{*}$; indeed, for those Species, once we order the points, there are no automorphisms. 
Now since generically, in the case of either of these excess divisors, at most two of the $b$ sections come together as one degenerates from an ordinary Hurwitz covering, it is clear that the ramification index is either 1 or 2 . Thus, to show that in both of these cases the ramification index is, in fact, 2 , it suffices to construct one example where $S=\operatorname{Spec} \mathbb{C}[[x]], C \rightarrow S$ a proper, smooth curve of genus $g$, with a diagram:

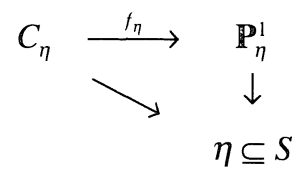

such that $f_{\eta}$ is an ordinary Hurwitz covering which generates to a covering of Species 1 or $3 B^{*}$ and such that if $T=S \times, \mathscr{H}^{\sim,+}$, then $T \rightarrow S$ is ramified.

$\$ 6.25$. We construct such examples using the theory of vanishing cycles reviewed in the Appendix to $\S 4$. Let $S=\operatorname{Spec} R$, with $R=\mathbb{C}[[x]], R_{t}=\mathbb{C}\left[\left[x^{i}\right]\right] \subseteq R$ for $i$ a natural number, $S_{t}=\operatorname{Spec} R_{t}$. Choose distinct nonzero complex numbers $\lambda_{2}, \ldots, \lambda_{b-1}$ and denote the corresponding sections of $\mathbb{P}^{1}$ by $\sigma_{2}, \ldots, \sigma_{b-1}$. We will later subject the complex numbers $\lambda_{t}$ to a further condition which the generic choice of $\lambda_{l}$ 's will fulfill (assuming $d$ to be large). By abuse of notation, we also denote by $\sigma_{2}, \ldots, \sigma_{b-1}$ the sections pulled back to $S_{1}, S_{2}$, and $S_{3}$. Write $\mathbf{C}[[T]]$ for the completed local ring at the point 0 of $\mathbb{P}_{\mathbf{C}}^{1}$. Let (for $\left.i=0,1\right) \sigma$, be the section of $\mathbb{P}_{S_{3}}^{1}$ which is the graph of the map $S_{3} \rightarrow \mathbb{P}_{\mathbf{C}}^{1}$ given by $T \mapsto(-1)^{\prime} X^{3}$. Let $\tau$ be the unique nontrivial element of the Galois group of $S_{3}$ over $S_{6}$. Then $\tau\left(\sigma_{t}\right)=\sigma_{1-t}$ for $i=0,1$, which $\tau\left(\sigma_{1}\right)=\sigma_{1}$ for $i \geq 2$. Denote by $\eta_{1}$ the generic point of $S_{1}$. Now if $G_{t}=\operatorname{Gal}\left(k(\bar{\eta}) / k\left(\eta_{1}\right)\right)$, where $k(\bar{\eta})=k\left(\bar{\eta}_{t}\right)$ for all $i$, then we have $G_{1} \subseteq G_{3} \subseteq G_{6}$, and $G_{1} \subseteq G_{2} \subseteq G_{6}$. Choosing a root of unity, we get isomorphisms $G_{1} \cong \mathbb{Z}^{\wedge}$ for all $i$. Let $\zeta_{1}$ be the canonical generator of $G_{1}$ under this isomorphism. Then $\zeta_{6}^{2}=\zeta_{3}$, $\zeta_{6}^{3}=\zeta_{2}, \zeta_{6}^{6}=\zeta_{1}$. Moreover $\zeta_{6}$ acts like $\tau$ on the $\sigma_{1}$ 's.

"Drawing" paths around the $\sigma_{i}$ 's, we see that the fundamental group $\Gamma$ of $\mathbb{P}_{\bar{\eta}}^{1}-\left\{\sigma_{0}, \sigma_{1}, \ldots, \sigma_{b-1}\right\}=U_{\bar{\eta}}$ may be described as the (pro-finite completion of the) free group on generators $g_{0}, g_{1}, \ldots, g_{b-1}$ modulo the sole relation $g_{0} g_{1} g_{2} \ldots g_{b-1}=1$. A simple calculation reveals the action of $\zeta_{6}$ on $\Gamma$ is given by $g_{0} \mapsto g_{0}$, $g_{1} \mapsto g_{1}^{-1} g_{0} g_{1}, g_{1} \mapsto g_{1}$ for $i \geq 2$. A connected étale covering of $U_{\bar{\eta}}$ of degree $d$ is defined by a transitive action of $\Gamma$ on the set $\{1, \ldots, d\}$ of $d$ elements. This covering is rational over $\eta_{t}$ if and only if the action of $\Gamma$ obtained by composing the original action with the automorphism of $\Gamma$ defined by $\zeta_{1}$ is the same as the original action. Finally, note that the subscheme $\sigma_{0} \cup \sigma_{1}$ of $\mathbb{P}_{S_{3}}^{1}$ descends to a 
closed, irreducible subscheme of $\mathbb{P}_{S_{6}}^{1}$, which, as an $S_{6}$-scheme, is isomorphic to $S_{3}$, hence ramified over $S_{6}$.

The Case of Species 1: Choose a transitive sequence of $b$ transpositions in $\mathscr{S}^{d}$ whose product is 1 to assign consecutively to $g_{0}, \ldots, g_{b-1}$, such that the first two transpositions are (12). We assume that the $\lambda_{t}$ 's are chosen such that the curve of genus $g$ in the special fibre over $S$ in the covering defined by this sequence of transpositions has no nontrivial automorphisms. (This will always be possible for $d>2 g+4$ by Proposition 5.) Clearly $\zeta_{6}$ acts trivially on this homomorphism from $\Gamma$ to $\mathscr{S}^{d}$, so we get the example desired over $S_{6}$.

The Case of Species 3B*: Choose a transitive sequence of transpositions as above that gives rise this time to Species $3 B^{*}$, with the first transposition being (12) and the second (23). Again we assume the $\lambda_{1}$ 's and sequence of transpositions chosen so that the curve of genus $g$ in the special fibre over $S$ has no nontrivial automorphisms. A simple calculation reveals that $\zeta_{2}$ acts trivially on the induced homomorphism from $\Gamma$ to $\mathscr{S}^{d}$, so we obtain the desired example over $S_{2}$.

We have thus proven the following

Proposition. $\quad \mu$ has ramification index 2 at divisors of Species 1 and $3 B^{*}$.

\$6.26. Corollary. Assuming still that $g \geq 5$ and $d>2 g+4$, $\mathscr{H} \mathscr{R} C_{b, d}$ and $\mathscr{H} U \overbrace{b, d}$ are geometrically connected.

Proof. We treat the rigidified case; the unrigidified case is analogous. If not, there would be a nontrivial maximal unramified extension between $\mathscr{H E R C}_{b, d}$ and $\mathscr{H} \mathscr{R} \mathscr{S}_{b . d}$, but our explicit computation of the ramification shows that this is impossible. The result thus follows from the corresponding result for $\mathscr{H} \mathscr{R} \mathscr{S}_{b, d}$ (§2).

Remark. The referee has informed me that this last result was obtained by Clebsch directly via purely combinatorial methods similar to those used in $\S 2$.

$\S 6.27$. In closing, we shall prove the following:

Proposition. $\mu$ is unramified over all odd primes $p$ of $\mathbb{Z}$.

Proof. As $\mathscr{G}$ is regular, it suffices to show, by the purity theorem, that there exists a geometric point of $\mathscr{H}$ of any odd characteristic such that the covering of this geometric point induced by $\mathscr{H}^{+} \rightarrow \mathscr{H}$ is étale. (Here we note that $\mathscr{H}$ can be 
defined over $\mathbb{Z}$ as the complement in $\mathscr{G}$ of the three divisors $\mathscr{Q}$, $\mathscr{Z}_{2}$, and $\mathscr{D}_{3}$, which are clearly defined and proper over $\mathbb{Z}$.) Translated, this means that given an algebraically closed field $k$ of odd characteristic $p$, we must find a smooth, proper, connected curve $C$ over $k$ and a morphism $f: C \rightarrow \mathbf{P}_{k}^{1}$ of degree $d$ such that the discriminant of $f$ is étale over $k$. But this clearly follows from the analysis of $\S 4$, which works so long as $k$ has odd characteristic. $\bigcirc$

Remark. It was the goal of this Subsection to determine the indices of ramification of $\mu$ at all the primes of height one of $\mathscr{G}$ (over $\mathbf{Z}$ ). We have thus completed this task at all primes except 2 , where there is wild ramification, thus making our goal at this prime beyond the scope of this paper.

\section{§7. The Coefficient Matrix}

$\S 7.1$. In this Section we shall complete the proof of Theorem 8 of $\S 4$ by showing that the excess divisors in $\mathscr{G}$ are linearly independent in $\operatorname{Pic}(\mathscr{G})$, and, in the process, we shall explicitly compute the coefficients of $\lambda, \Theta$, and $\gamma$ in terms of $\mathscr{D}, \mathscr{D}_{2}$, and $\mathscr{B}_{3}$. This also gives us explicitly the "main filtration" (\$5.10) in terms of the $\mathscr{D}_{1}$ 's. We can thus check the coefficients of $\lambda$ and $\Theta$ by making sure that they are in the kernel of the map $F^{3} \rightarrow F^{3} / F^{2} \cong \mathbf{Q} \delta_{G}$, which was computed explicitly in $\S 6 \mathrm{~B}$. As usual, it suffices to work over the complex field $\mathbf{C}$, so we will do that in this Section.

$\S 7.2$. Let us note, first of all, that there exists a proper, smooth, connected curve $S$ (over $\mathbf{C}$ ) with the following property: there exist enough morphisms $\phi_{1}: S \rightarrow \overline{\mathscr{H}}^{+}$that avoid nonexcess divisors so that if we write $\psi_{1}: S \rightarrow \mathscr{G}$ for the morphism induced by composing $\phi_{1}$ with $\kappa$, then the matrix $\left\{\operatorname{deg}\left(\psi_{1}, \mathscr{F}_{j}\right)\right\}$, where $j$ $=1,2,3 ; \mathscr{F}_{1}=\lambda ; \mathscr{F}_{2}=\Theta ; \mathscr{F}_{3}=\gamma$, is of rank three. Indeed, we can prove the existence of such an $S$ as follows. First, take any nonconstant morphism $f^{\prime}: S^{\prime} \rightarrow \mathscr{M}_{g}$, where $S^{\prime}$ is a proper, smooth, connect curve (over $\mathbf{C}$ ). (To see the existence of such an $f^{\prime}$, consider the Satake embedding $M_{g} \hookrightarrow \mathbf{P}^{N}$ of the coarse moduli space $M_{g}$; since the divisors at infinity of $\overline{\mathscr{M}}_{g}$ map to subsets of codimension $\geq 2$ in the closure $\bar{M}_{g}$ of $M_{g}$ in $\mathbf{P}^{N}$, one can construct a proper curve inside $M_{g}$ by cutting by hyperplane sections.) Let $G^{\prime}=\mathscr{G} \times{ }_{1 /} S^{\prime}$. Now it follows from our explicit analysis of $\operatorname{Pic}_{\mathbf{Q}}(\mathscr{G})$ that the natural morphism $\operatorname{Pic}_{\mathbf{Q}}(\mathscr{G}) \rightarrow \operatorname{Pic}_{\mathbf{Q}}\left(G^{\prime}\right)$ is injective. Moreover, it follows from Proposition 5 of $\S 4$ that the inverse images via $G^{\prime} \rightarrow \mathscr{G}$ of the images via $\mu$ of the nonexcess divisors are of codimension at least two in $G^{\prime}$. Thus, it suffices to prove the original statement for $G^{\prime}$ instead of $\mathscr{G}$; but since $G^{\prime}$ is clearly a projective scheme, the existence of $S$ and the $\psi$, follows by reducing to the case when $G^{\prime}$ is a smooth, 
proper surface (by cutting with hyperplane sections) and then taking a basis of the $\mathbb{Q}$-Néron-Severi group of the surface which consists of smooth very ample divisors. The only remaining technical point is that it may at first seem that we ned to use different curves $S_{l}$ with $\psi_{t}: S_{l} \rightarrow G^{\prime}$ (or $\mathscr{G}$, or $\overline{\mathscr{H}}^{+}$), but in fact, it is elementary that given any finite number of curves $S_{1}$, there exists a curve $S$ and finite morphisms $\alpha_{1}: S \rightarrow S_{1}$. This completes the proof.

Let us fix one such morphism $\phi: S \rightarrow \overline{\mathscr{H}}^{+}$. Now let us note that if we can prove a linear relation between the degrees of the pull-backs via $\psi$ of certain line bundles and divisors from $\mathscr{G}$ for all $\phi$ as above, then the relation must, in fact, hold on $\mathscr{G}$.

Now $\phi$ corresponds to some admissible covering $f^{\sim}: X^{\sim} \rightarrow P^{\sim}$ over $S$. Let us denote by $\sigma_{0}, \ldots, \sigma_{b-1}$ the sections $S \rightarrow P^{\sim}$ that make up the discriminant of $f^{\sim}$. The stable curve associated to $p^{\sim}: X^{\sim} \rightarrow S$, which we shall call $p: X \rightarrow S$ (obtained as in \$3.27), is, in fact, smooth, and the only kinds of degeneracies that occur are those of Species 1,2, or $3 B^{*}$. Let us denote by $U \subseteq X$ the complement of the finite set of points that are the points of degenerate ramification in the fibres of Species 3B* (so there is exactly one such point in each fibre of Species $\left.3 \mathrm{~B}^{*}\right)$. Then one sees easily that $f^{\sim}$ contracts to a map $f: U \rightarrow P$, where $P$ is a $\mathbb{P}^{1}$-bundle over $S$. Thus we have a diagram:

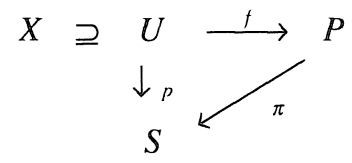

By abuse of notation, we also denote by $\sigma$, the push-forwards of the original $\sigma_{1}$ to sections of $\pi$. We denote by $\tau_{t}$ the unique section of $X \rightarrow S$ that lies over $\sigma_{t}$ and passes through the points of ramification. We denote by $\widetilde{C}_{P}(1)$ some line bundle on $P$ (which we fix for the entire discussion, but the choice of which is noncanonical) whose negative square is the relative canonical bundle for $P \rightarrow S$. (Note that we may need to replace $S$ by some finite étale cover of $S$ in order for ${ }_{P}(1)$ to exist.) Observe that the square of the first Chern class of $c_{P}(1)$ is zero. Let $\mathcal{L}^{\prime}$ be the unique line bundle on $X$ such that, restricted to $U$, it is equal to $f \overbrace{P}(1)$. By abuse of notation, we will similarly write $f \mathscr{F}$ for other line bundles $\mathscr{F}$ on $P$ to denote the unique extension to $X$ of the actual bundle $f^{\prime} \mathscr{F}$ on $U$. Also, let $R=\sum_{l=0}^{b-1} \tau_{l}$, in the sense of divisors. Then we see that $\omega_{X}=$ $\left(p \omega_{S}\right) \otimes\left(f \omega_{P / S}\right)(R)$ and $\omega_{X / S}=f^{\prime} \omega_{P / S}(R)$, where the various $\omega$ 's denote the respective canonical bundles. 
Let $h_{l}$ denote the height of the section $\sigma_{l}$ of $\pi$ relative to $C_{P}(1)$. Thus, on $P$, we have $\sigma_{l} \cdot \sigma_{J}=h_{t}+h_{J}$. Let $l=\sum h_{t}$. Let us choose two distinct, constant, but sufficiently generic divisors $F_{1}$ and $F_{2}$ on $P$ which are of the form "the sum of a divisor which is a (sufficiently generic) section of $P \rightarrow S$ and various (sufficiently generic) vertical divisors" and which is such that $C_{P}(1) \cong C_{P}\left(F_{1}\right) \cong C_{P}\left(F_{2}\right)$. Let $G_{i}$ be the divisor $f^{-1} F_{1}$ on $X$, for $i=1,2$, so that $\mathscr{Z}^{\prime} \cong C_{X}\left(G_{1}\right) \cong C_{X}\left(G_{2}\right)$.

$\S 7.3$. We wish to compute the following intersection numbers of divisors on $X$ :
(A) $G_{1}^{2}=G_{2}^{2}=G_{1} \cdot G_{2}$
(B) $\tau_{1} \cdot G_{1}=\tau_{1} \cdot G_{2}$
(C) $\tau_{1} \cdot \tau$, (for $i \neq j$ )
(D) $\tau_{t}^{2}$

It is clear that it suffices to work locally over $S$, i.e., using local intersection theory. Thus we let $E=\operatorname{Spec} A$ with $A$ the completion of the local ring at some closed point $x$ of $S$ and obtain a diagram

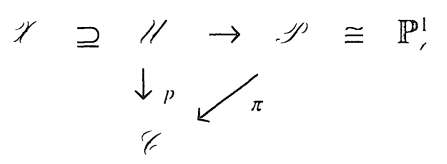

which is the result of applying the base change $\% \rightarrow S$ to the diagram in the previous $\S$. By abuse of notation, we continue to use the symbols $\sigma_{1}, \tau_{1}$ for the base-changed sections. Also note that it suffices to compute the result in the

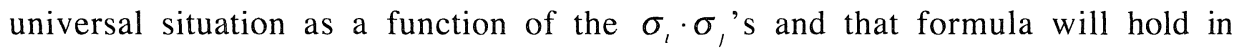
general.

Finally, note that (in the global situation) if $q=$ the genus of $S$, then by the adjunction formula, we have:

$$
\begin{aligned}
2(q-1) & =\tau_{1}^{2}+\tau_{1} \cdot \omega_{X} \\
& =\tau_{1}^{2}+\tau_{1}^{2}+\tau_{1} \cdot\left(R-\tau_{1}\right)+\tau_{1} \cdot p \omega_{S}+\tau_{1} \cdot f \omega_{P / s} \\
& =2 \tau_{1}^{2}+\tau_{1} \cdot\left(R-\tau_{1}\right)+2(q-1)-2 \tau_{1} \cdot G_{1}
\end{aligned}
$$

Thus, $\tau_{1}^{2}=-\frac{1}{2} \tau_{1} \cdot\left(R-\tau_{1}\right)+\tau_{1} \cdot G_{1}$. Hence it suffices to compute (A), (B), and (C) locally.

\$7.4. We begin with the case of a closed point $x \in S$ where the covering does not degenerate. Then it is clear that: 

(A) $G_{1} \cdot G_{2}=0$
(B) $\tau_{l} \cdot G_{1}=0$ for most points $x$, except for a finite number of special points; the sum of the local intersections over these points is $h_{t}$.
(C) $\tau_{t} \cdot \tau_{j}=0$ for $i \neq j$.

$\S 7.5$. Equally simple is the case of Species 2 . Here we see that:
(A) $G_{1} \cdot G_{2}=0$
(B) $\tau_{1} \cdot G_{1}=0$ (since $G_{1}$ is sufficiently generic)
(C) $\tau_{1} \cdot \tau_{j}=0$ for $i \neq j$.

\$7.6. We now consider the case of Species 1. By reduction to the universal case, we may assume that $\sigma_{t} \cdot \sigma_{j}$ (where $i \neq j$ ) is 0 except when $\{i, j\}=\{0,1\}$ in which case $\sigma_{0} \cdot \sigma_{1}=3$. Now we have the following formulas for divisors:

$$
\begin{aligned}
& f^{-1}\left(\sigma_{0}\right)=2 \tau_{0}+E_{0} \\
& f^{-1}\left(\sigma_{1}\right)=2 \tau_{1}+E_{1}
\end{aligned}
$$

where $E_{0}, E_{1}$ are effective horizontal divisors of $\mathscr{X}$ of degrees $d-2$ over $\mathscr{C}$. Thus we get: $3=\sigma_{0} \cdot \sigma_{1}=f_{*}\left(\tau_{0}\right) \cdot \sigma_{1}=\tau_{0} \cdot f^{-1}\left(\sigma_{1}\right)=2 \tau_{0} \cdot \tau_{1}+\tau_{0} \cdot E_{1}$. But it is clear that $\tau_{0} \cdot \tau_{1}$ and $\tau_{0} \cdot E_{1}$ are both positive integers. Thus $\tau_{0} \cdot \tau_{1}=1$. Returning to the general local case of Species 1, we see that if for some $m, k, \sigma_{m} \cdot \sigma_{k} \neq 0$ while $\sigma_{\imath} \cdot \sigma_{J}=0$ for all other combinations, then:
(A) $G_{1} \cdot G_{2}=0$
(B) $\tau_{t} \cdot G_{1}=0$ (since $G_{1}$ is sufficiently generic)
(C) $\tau_{m} \cdot \tau_{k}=\frac{1}{3} \sigma_{m} \cdot \sigma_{k}, \tau_{t} \cdot \tau_{j}=0$ for all other combinations $\{i, j\}$.

\$7.7. The case of Species $3 B^{*}$ is somewhat more involved. Let us first note that by reduction to the universal case, we may assume that $\sigma_{0} \cdot \sigma_{1}=1$, and $\sigma_{1} \cdot \sigma_{j}=0$ (for $i \neq j$ ) for all other combinations. Then if we define $\mathscr{X}^{-}$to be the scheme obtained from $\mathscr{E}$ as a result of two ordinary blow-ups at points taken in a certain fashion, then $f$ will extend to a morphism $f^{\sim}: \mathscr{X}^{\sim} \rightarrow \mathscr{P}^{\sim}$ which is everywhere defined and is the pull-back via $\mathscr{E} \rightarrow S$ of the $f^{\sim}$ of $\S 7.2$. Let $\mathscr{X}_{1}$ be the blow-up of $\mathscr{X}$ at the point of degenerate simple ramification. Denote by $D$ the exceptional

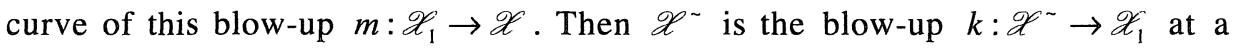


point of $D$. Let $C_{1}$ be the strict transform of $D$ in $\mathscr{X}^{\sim}$, and $C_{2}$ the exceptional curve of $k$. Let $h=m \circ k$. Then we have a diagram:

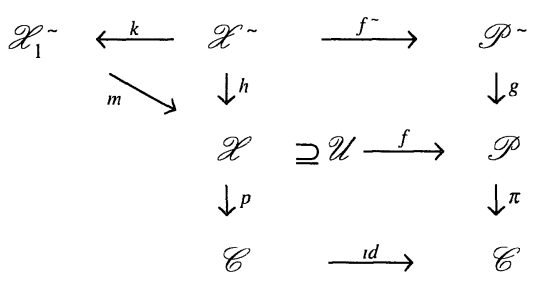

Moreover we have the sections $\sigma_{\imath}$ of $\pi$, and their unique liftings $\sigma_{i}^{\sim}$ to sections of $\pi \circ g$; on the other hand, we also have the sections $\tau_{t}$ of $p$ lifting the $\sigma_{t}$, the sections $\tau_{1}^{(1)}$ of $p \circ m$ lifting the $\tau_{1}$, and the sections $\tau_{1}^{\sim}$ of $p \circ h$ again lifting the $\tau_{1}$. Let (for $\left.i=1,2\right) G_{1}^{(1)}$ and $G_{1}^{\sim}$ be the respective strict transforms on $\mathscr{X}_{1}$ and $\mathscr{X}^{\sim}$. Let $\mathscr{L}^{(1)}=m^{4} \mathscr{L}, \mathscr{L}^{\sim}=h^{\prime} \mathscr{L}=k^{\prime} \mathscr{L}^{(1)}, \mathscr{M}=g^{\prime} \mathscr{O}_{,}(1), \mathscr{M}^{\sim}=f^{\sim, 1} \mathscr{M}$. Now $\mathscr{C}_{\mu}(1) \cdot \mathscr{O}_{\mu}(1)=0$, so $\mathscr{M} \cdot \mathscr{M}=0$, and $\mathscr{M}^{\sim} \cdot \mathscr{M}^{\sim}=0$. But clearly, $\mathscr{M}^{\sim}=\mathscr{O}_{\gamma^{-}}\left(G_{1}^{\sim}\right)$ $=\mathscr{O}_{,},\left(G_{2}^{\sim}\right)$, so $\left(G_{1}^{\sim}\right)^{2}=\left(G_{2}^{\sim}\right)^{2}=G_{1}^{\sim} \cdot G_{2}^{\sim}=0$. On the other hand, $k^{4} D=C_{1}+C_{2}$ and $D^{2}=-1, \quad C_{2}^{2}=-1, C_{1} \cdot C_{2}=1$, so we get $-1=C_{1}^{2}+2 C_{1} \cdot C_{2}+C_{2}^{2}$, hence $C_{1}^{2}=-2$. Also, it is clear that for $i=1,2$, we have $G_{1}^{-} \cdot C_{1}=0$ and $G_{l}^{\sim} \cdot C_{2}=1$. Now $\mathscr{L}^{(1)}=\mathscr{C}\left(G_{1}^{(1)}+D\right)$, so that $\mathscr{L}^{\sim}=\mathscr{G}\left(G_{1}^{\sim}+C_{1}+2 C_{2}\right)$, where $i=1,2$. Hence,

$$
\begin{aligned}
G_{1} \cdot G_{2} & =\mathscr{L} \cdot \mathscr{L}=\mathscr{L}^{\sim} \cdot \mathscr{L}^{\sim} \\
& =\left(G_{1}^{\sim}+C_{1}+2 C_{2}\right) \cdot\left(G_{2}^{\sim}+C_{1}+2 C_{2}\right) \\
& =4+\left(C_{1}+2 C_{2}\right)^{2}=4-2-4+4=2
\end{aligned}
$$

Let us now note the following easy relations: $\tau_{0}^{\sim} \cdot \tau_{1}^{\sim}=0, \tau_{1}^{\sim} \cdot C_{1}=1, \tau_{1}^{\sim} \cdot C_{2}=0$, $\tau_{i}^{\sim} \cdot G_{j}^{\sim}=0$ for all $i \in\{0,1\}, j \in\{1,2\}$. Also, we see that $h^{\prime} \tau_{t}=\tau_{t}^{\sim}+C_{1}+C_{2}$, for $i \in\{0,1\}$. Thus,

$$
\begin{aligned}
\tau_{0} \cdot \tau_{1} & =h^{\prime} \tau_{0} \cdot h^{\prime} \tau_{1} \\
& =\left(\tau_{0}^{\sim}+C_{1}+C_{2}\right) \cdot\left(\tau_{1}^{\sim}+C_{1}+C_{2}\right) \\
& =2+\left(C_{1}+C_{2}\right)^{2}=2-1=1
\end{aligned}
$$

Finally, for any $i \in\{0,1\}, j \in\{1,2\}$,

$$
\begin{aligned}
\tau_{1} \cdot G_{\jmath} & =h^{\prime}\left(\tau_{l}\right) \cdot h^{\prime}\left(G_{J}\right) \\
& =\left(\tau_{1}^{\sim}+C_{1}+C_{2}\right) \cdot\left(G_{J}^{\sim}+C_{1}+2 C_{2}\right)=1+2-2=1
\end{aligned}
$$


Returning to the general local case of Species $3 B^{*}$, we see that if $\sigma_{m} \cdot \sigma_{h} \neq 0$ for some $m, k$ and $\sigma_{i} \cdot \sigma_{j}=0$ for all other combinations of $i, j$ with $i \neq j$, then:

(A) $G_{1} \cdot G_{2}=2 \sigma_{m} \cdot \sigma_{k}$

(B) $\tau_{l} \cdot G_{1}=\sigma_{m} \cdot \sigma_{h}$ for $i \in\{m, k\}, 0$ otherwise.

(C) $\tau_{m} \cdot \tau_{h}=\sigma_{m} \cdot \sigma_{h}, \tau_{t} \cdot \tau_{\jmath}=0$ for all other combinations such that $i \neq j$.

$\S 7.8$. We are now ready to return to the global situation of $\$ 7.2$ and compute global intersection numbers. Let $v_{1}$ (resp. $v_{2}, v_{3}$ ) denote the number of points of $S$ (with multiplicity) at which a degeneracy of Species 1 (resp. 2, 3B*) occurs. Thus $v_{1}+v_{2}+v_{3}=l(b-1)$. First, we see that $\mathscr{L} \cdot \mathscr{L}=G_{1} \cdot G_{2}=2 v_{3}$; hence $\left(f^{\prime} \omega_{P / S}\right)^{2}=8 v_{3}$. Next, we have $R \cdot \mathscr{Z}=\sum_{l=o}^{b-1} \tau_{1} \cdot G_{1}=l+2 v_{3}$; hence $R \cdot\left(f^{\prime} \omega_{P / S}\right)=$ $-2\left(l+2 v_{3}\right)$. Finally, we have:

$$
\begin{aligned}
R^{2} & =\sum_{l=0}^{b-1} \tau_{l}^{2}+2 \sum_{i<J} \tau_{t} \cdot \tau_{J} \\
& =\sum_{l=0}^{b-1} \tau_{l}^{2}+\frac{1}{2}\left(\sum_{l \neq j} \tau_{l} \cdot \tau_{\jmath}\right)+\left(\sum_{i<j} \tau_{l} \cdot \tau_{J}\right) \\
& =\sum_{l=0}^{b-1}\left\{\tau_{l}^{2}+\frac{1}{2} \tau_{l} \cdot\left(R-\tau_{l}\right)\right\}+\sum_{i<j} \tau_{l} \cdot \tau_{J} \\
& =R \cdot G_{1}+\frac{1}{3} v_{1}+v_{3} \\
& =l+\frac{1}{3} v_{l}+3 v_{3}
\end{aligned}
$$

We have, after possibly enlarging $S$, a morphism $\alpha: S \rightarrow \xi$ corresponding to the diagram in $\S 7.2$. Let $\delta_{t}=\operatorname{deg}\left(\alpha \mathscr{Z}_{t}\right)$. Then by $\S 6 \mathrm{C}$, we see that $v_{1}=\frac{3}{2} \delta_{1}$, $v_{2}=\delta_{2}, v_{3}=\frac{1}{2} \delta_{3}$. Thus we summarize as follows:

(1) $3 \delta_{1}+2 \delta_{2}+\delta_{3}=2 l(b-1)$

(2) $\left(f \omega_{P / S}\right)^{2}=4 \delta_{3}$

(3) $R \cdot\left(f \omega_{P / S}\right)=-2\left(l+\delta_{3}\right)$

(4) $R^{2}=l+\frac{1}{2} \delta_{1}+\frac{3}{2} \delta_{3}$. 
$\S 7.9$. Let us compute $\lambda_{S}=\frac{1}{12}\left(\omega_{X / S}\right)^{2}$ purely in terms of $\delta_{1}, \delta_{2}$, and $\delta_{3}$ (and not l). We have:

$$
\begin{aligned}
\omega_{X / S}^{2} & =\left(f^{\prime} \omega_{P / S}\right)^{2}+R^{2}+2 R \cdot\left(f^{\prime} \omega_{P / S}\right) \\
& =-3 l+\frac{1}{2} \delta_{1}+\frac{3}{2} \delta_{3} \\
& =\frac{1}{2(b-1)}\left\{-3\left(3 \delta_{1}+2 \delta_{2}+\delta_{3}\right)+(b-1)\left(\delta_{1}+3 \delta_{3}\right)\right\} \\
& =\frac{1}{2(b-1)}\left\{(b-10) \delta_{1}-6 \delta_{2}+3(b-2) \delta_{3}\right\}
\end{aligned}
$$

Now by the reasoning of $\$ 7.2$, by choosing various $\phi$, we obtain enough possibilities so that any relation among line bundles on $\mathscr{G}$ that holds for all these possibilities must, in fact, hold on $\mathscr{G}$. Thus we obtain the following:

Proposition. $\quad \lambda=\frac{1}{24(b-1)}\{(b-10) \mathscr{Z}-6 \mathscr{Z} / 2+3(b-2) \mathscr{L}\}$.

$\$ 7.10$. It follows from the definition and from [26], Corollary 2.7.6 of Exposé II, that $\Theta_{S}=-\frac{1}{2}\left(\mathscr{Z}-\frac{d}{2(g-1)} \omega_{X / S}\right)^{2}$. Let $\chi=2(g-1)$. Then we see that $\left(-8 \chi^{2}\right) \Theta_{S}=\left(\chi R-b \omega_{X / S}\right)^{2}$. Note that $\chi=b-2 d$. Then

$$
\begin{aligned}
(-8 \chi) \Theta_{S}= & (b-2 d) R^{2}-2 b R \cdot\left(\omega_{X / S}\right)+\left(\frac{b^{2}}{\chi}\right)\left(\omega_{X / S}\right)^{2} \\
= & -(b+2 d)\left(l+\frac{1}{2} \delta_{1}+\frac{3}{2} \delta_{3}\right)+4 b\left(l+\delta_{3}\right) \\
& +\frac{b^{2}}{2(b-2 d)(b-1)}\left\{(b-10) \delta_{1}-6 \delta_{2}+3(b-2) \delta_{3}\right\}
\end{aligned}
$$

Thus,

$$
\begin{aligned}
-16 \chi(b-1) \Theta_{s}= & (3 b-2 d)\left(3 \delta_{1}+2 \delta_{2}+\delta_{3}\right)-(b+2 d)(b-1) \delta_{1}+(b-1)(5 b-6 d) \delta_{3} \\
& +\frac{b^{2}}{(b-2 d)}\left\{(b-10) \delta_{1}-6 \delta_{2}+3(b-2) \delta_{3}\right\} \\
= & -\{b(b-10)+2 d(b+2)\} \delta_{1}+2(3 b-2 d) \delta_{2}+\{b(5 b-2)-2 d(3 b-2)\} \delta_{3} \\
& +\frac{b^{2}}{(b-2 d)}\left\{(b-10) \delta_{1}-6 \delta_{2}+3(b-2) \delta_{3}\right\}
\end{aligned}
$$

Thus, 


$$
\begin{aligned}
-16(b-1)(b-2 d)^{2} \Theta_{S}= & \left\{b^{2}(b-10)-b(b-2 d)(b-10)-2 d(b-2 d)(b+2)\right\} \delta_{1} \\
& +\left\{2(3 b-2 d)(b-2 d)-6 b^{2}\right\} \delta_{2} \\
& +\left\{b(5 b-2)(b-2 d)-2 d(3 b-2)(b-2 d)+3 b^{2}(b-2)\right\} \delta_{3} \\
= & -4 d\{6 b-d(b+2)\} \delta_{1}+8 d(d-2 b) \delta_{2}+4\left\{2 b^{2}(b-1)\right. \\
& \left.-2 b d(2 b-1)+d^{2}(3 b-2)\right\} \delta_{3}
\end{aligned}
$$

By the same logic as above,

Proposition. $\quad \Theta=\frac{1}{4(b-1)(b-2 d)^{2}}\left(d\{6 b-d(b+2)\} \mathscr{Q}-2 d(d-2 b) \mathscr{Z _ { 2 }}\right.$ $\left.\left\{2 b^{2}(b-1)-2 b d(2 b-1)+d^{2}(3 b-2)\right\} \mathscr{Q}\right)$

$\S 7.11$. Let $\mathscr{E}=p \mathscr{L}$. Then $\mathscr{E}$ is a vector bundle of rank $d+1-g$ on $S$. Noting by Riemann-Roch that $\pi, \mathscr{C}_{p}(1)$ has degree zero, we see that it follows from the definitions that $(d+1-g) \gamma_{S}=2 N$, where $N=\operatorname{deg}(\mathscr{E})$. Let $\kappa=c_{1}\left(\omega_{X / S}\right)$, $\mu=c_{1}(\mathscr{L})$. By Riemann-Roch,

$$
\begin{aligned}
N & =\operatorname{Td}(-\kappa) \operatorname{ch}(\mu)=\left(1-\frac{\kappa}{2}+\frac{\kappa^{2}}{12}\right)\left(1+\mu+\frac{1}{2} \mu^{2}\right) \\
& =\frac{\kappa^{2}}{12}+\frac{\mu^{2}}{2}-\frac{\kappa \mu}{2}
\end{aligned}
$$

In our language, then,

$$
\begin{aligned}
24 N & =2\left(\omega_{X / S}\right)^{2}+3\left(f \omega_{P / S}\right)^{2}+6 \omega_{X / S} \cdot\left(f \omega_{P / S}\right) \\
& =2\left(\omega_{X / S}\right)^{2}+9\left(f^{\prime} \omega_{P / S}\right)^{2}+6 R \cdot\left(f^{\prime} \omega_{P / S}\right)
\end{aligned}
$$

Hence,

$$
\begin{aligned}
24(b-1) N & =(b-10) \delta_{1}-6 \delta_{2}+3(b-2) \delta_{3}+\left\{36 \delta_{3}-12 \delta_{3}-12 l\right\}(b-1) \\
& =(b-10) \delta_{1}-6 \delta_{2}+3\{(b-2)+8(b-1)\} \delta_{3}-6\left(3 \delta_{1}+2 \delta_{2}+\delta_{3}\right) \\
& =(b-28) \delta_{1}-18 \delta_{2}+9(3 b-4) \delta_{3}
\end{aligned}
$$

Thus we have the following:

$$
\text { Proposition } \gamma=\frac{1}{6(b-1)(4 d-b)}\left\{(b-28) \mathscr{D}_{1}-18 \mathscr{L}_{2}+9(3 b-4) \mathscr{B}_{3}\right\}
$$

\$7.12. Remark. This completes our calculation. As remarked previously, we can check our calculation by making sure that the expressions for $\lambda$ and $\Theta$ go to 
zero, while that for $\gamma$ goes to one under the map: $\mathscr{Z}_{1} \mapsto 3(b-d)$, $\mathscr{\mathscr { L }}_{2} \mapsto \frac{b}{2}(b-10)+4 d, \mathscr{\mathscr { B }}_{3} \mapsto d$.

$\S 7.13$. We are now in a position to complete the proof of Theorem 6 of $\S 6$, and hence of its consequences, first mentioned in $\S 4$, and restated in $\$ 6.7$. We wish to show that $\mathscr{R}, \mathscr{\mathscr { Z }}$, and $\mathscr{\mathscr { Z }}$ are linearly independent in the Picard group of $\mathscr{G}$. But from the relations obtained already in this Section, we see that the $\mathbf{Q}$-linear subspace generated by the excess divisors already contains a basis for the whole space. The result thus follows immediately.

\section{§8. Arithmetic Applications}

$\S 8.1$. In this Section, we make suggestions about possible applications of the material discussed up till now to giving

(1) An alternate proof of the Shafarevich conjecture

(2) An effective version of the Mordell conjecture in the style of [30].

In particular, we expose a certain kind of argument discovered by the author that allows one to conclude the above arithmetic results by using the geometry of the compactification of the Hurwitz scheme. The argument to be exposed, however, has a couple of gaps that the author has not been able to fill in. We state the facts necessary to fill in these gaps as "Wishes," in the hope that perhaps some reader will see how to prove them, thus completing the proposed proofs of the above arithmetic results.

Let $B=\operatorname{Spec} c_{F}$, where $C_{F}$ is the ring of integers of a number field $F$. Let $f: C \rightarrow B$ be a smooth curve of genus $g \geq 3$. (We assume good reduction to simplify the discussion, since this case already illustrates the essential problem involved.)

§8.2. If $f$ is hyperelliptic (i.e., the geometric fibres of $f$ are hyperelliptic), then we can canonically write $f$ as a double covering of $\mathbb{P}_{B}^{1}$, so that to every such $f$ we can canonically assign (up to automorphisms of $\mathbf{P}_{B}^{1}$ ) a certain number of points of $\mathbb{P}_{B}^{1}$ which are distinct from each other outside primes over 2 . We can then conclude the "Shafarevich conjecture" for such hyperelliptic curves, i.e., that only a finite number of such curves exist over $B$, by applying Siegel's theorem on integral points. 
If $f$ is not hyperelliptic, then no such canonical presentation as a double covering exists. Generically, that is, over $F$, we can write $f$ as a Hurwitz covering of $\mathbb{P}_{F}^{1}$, but then the problem is that we can't apply Siegel's theorem unless we know that the points of ramification remain distinct, not only generically, but at all primes outside some fixed finite set. Thus it is natural to study in detail degenerations of Hurwitz coverings, i.e., the compactification of the Hurwitz scheme, to see if one can get around this problem. This was the original motivation for this paper.

§8.3. We can add some canonicality to the situation if we insist that we only consider coverings $f: C_{F} \rightarrow \mathbb{P}_{F}^{1}$ such that $f^{\prime} \mathscr{C}(1)=\omega_{C_{F} / F}^{\otimes N}$, where $N$ is some fixed integer. If we let $\sigma: \mathscr{M l}_{g} \rightarrow \mathscr{J}^{N \chi}$ be the section corresponding to $\omega_{C_{F} / F}^{\otimes N}$, and let $\mathscr{K}$ be such that the following diagram is Cartesian:

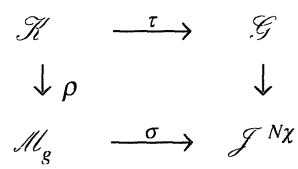

then ideally, we would like a section $\zeta$ of $\rho$ that avoids the $\mathscr{E}_{1}=\tau^{\prime} \mathscr{Z}_{1}$, for $i=1$, 2,3 . This is unrealistic, however. In fact, even constructing any section $\zeta$ at all is rather difficult.

At the other extreme, if we assume that the height (e.g. [10]) of our curve is bounded, then everything becomes trivial. Indeed, if we fix an appropriate metric on $\mathscr{\mathscr { K }}$ so as to give it an "integral structure" over the "compact curve" Spec $c_{F} \cup S_{\infty}$ (where $S_{\infty}=$ the archimedean places), then we see that if $c: B \rightarrow$ $\mathscr{M}_{\mathrm{g}}$ is the classifying map of our curve, then the number of possibilities for the Grassmann bundle $c^{\prime} \mathscr{K}$ is bounded as soon as $\operatorname{deg}_{A t}(c \lambda)$ (where " $\operatorname{deg}_{A^{\prime}}$ " means the degree of the arithmetic line bundle) is bounded, by the following result of Faltings:

Lemma. The number of closed subvarieties $V \subseteq \mathbb{P}_{B}^{M}$ (where $M$ is any positive integer), with $\pi: V \rightarrow B$ flat, of degree $d(\in \mathbb{N})$ as a subvariety over $F$ of $\mathbb{P}_{F}^{M}$, of relative dimension $n$ over $B$, and of height $\operatorname{deg}_{A_{1}}\left(\pi[\lessdot(1)]^{n+1}\right) \leq M^{\prime}($ for some fixed $M^{\prime} \in \mathbb{R}$ ) is finite.

Proof. (Sketch) The Lemma is obvious for hypersurfaces. But then one can easily reduce to the case of hypersurfaces via the method of the Chow variety. $O$ 
(Here we apply the Lemma by using the Plücker embedding for $c^{\prime} \mathscr{K}$ and note that

$$
\operatorname{deg}_{A^{\prime}} c^{\prime}\left(\rho_{r}[\gamma]^{N+1}\right) \leq K \operatorname{deg}_{A \prime}\left(c^{\prime} \lambda\right)
$$

for some fixed $K$ (independent of $c$ ) and for $N=$ relative dimension of $\rho$ ). Similarly, since the total number of possibilities for the entire situation is finite, we can certainly find some finite set $S$ of primes of $B$ such that we can always write curves $C$ with bounded height as Hurwitz coverings over $B-S$. (In fact, the Lemma also shows that the number of possibilities for $C$ is, in this case, also finite!).

§8.4. The problem, then, is to find an intermediate course. First, we must bound the number of possibilities for the Grassmann bundle $c^{\prime} \mathscr{K}$ without bounding $\operatorname{deg}_{A \prime}\left(c^{\prime} \lambda\right)$. If the Grassmann bundle is associated to a vector bundle $\mathscr{E}$ which is "sufficiently indecomposable" in the sense that it is parametrized by some sort of finite "cohomology group" on the compactified curve $\operatorname{Spec} c_{F} \cup S_{\infty}$ (cf. the wellknown situation for indecomposable vector bundles of rank 2 on curves over a finite field), then the number of possibilities for $c^{\prime} \mathscr{K}$ will be finite. At present, however, the author does not know how to prove this sort of result, so we merely state it as a "wish:"

Wish 1. The number of possibilities for c' $\mathscr{K}$ (as a Grassmann bundle) is finite.

Moreover, in order to be able to prove the Shafarevich conjecture as in the hyperelliptic case, we also need the following result:

Wish 2. Let $F$ be a number field, with ring of integers $c_{F}$. Let $\mathscr{G} \rightarrow \operatorname{Spec} \kappa_{F}$ be a Grassmann bundle with integral structure at infinity. Let $r \in \mathbf{N}$, $d_{1}, \ldots, d, \in \mathbb{N}$. Fix a finite set of closed primes $S \subseteq \operatorname{Spec} /_{F}$. Then there exists a finite set of closed primes $(S \subseteq) T \subseteq \operatorname{Spec} c_{F}$ with the following property:

(*) Let $\mathscr{Y}, \ldots, \mathscr{G} \subseteq \subseteq$ be flat divisors over Spec $C_{F}$ with $\mathscr{Z}_{1}$ of degree $d_{1}$ for all $i=1, \ldots, r$ that are geometrically integral and distinct outside $S$. Then there exists a section $\sigma: \operatorname{Spec} \tau_{F} \rightarrow \mathscr{G}$ that avoids $\mathscr{Z}_{1}, \ldots, \mathscr{Z}_{i}$ outside $T$.

The result is known to be true in the following cases:

(1) If $\%$ is a $\mathbb{P}^{1}$-bundle, then it follows from Siegel's theorem on integral points. 
(2) If $\mathscr{G}$ is a $\mathbb{P}^{2}$-bundle, then if we assume that the $\mathscr{D}_{1}$ are not only geometrically integral, but smooth, then it follows from the Shafarevich conjecture applied to smooth plane curves.

In both these cases, however, one should note that Siegel's theorem and the Shafarevich conjecture are somewhat stronger than Wish 2, and so using them to prove Wish 2 is sort of overkill. At this point, the author knows of no way to prove Wish 2 in any generality, but if a proof could be given for Wish 1 and Wish 2 , then one could prove the Shafarevich conjecture using the technique of Hurwitz coverings.

In closing we remark that since the Shafarevich conjecture is, in fact, known [10], the number of possibilities for $f: C \rightarrow B$ is, in fact, finite, so we can, indeed, find a fixed, finite set of primes $T$ which is independent of $F$ and such that we can write any $f: C \rightarrow B$ as a Hurwitz covering outside $S$. Thus, we see that something like Wish 1 and Wish 2 must be true, but to approach things in this way is to defeat the purpose of this venture!

$\S 8.5$. We now move on to motivating the possible application to an effective form of the Mordell conjecture. In $\$ 7$, we computed the coefficients of $\lambda$ in terms of the $\mathscr{D}_{1}$ and found that the coefficients for $\mathscr{Z}_{1}$ and $\mathscr{D}_{3}$ were positive, while that for $\mathscr{Z}_{2}$ was negative. Suppose, however, just for the moment that all three coefficients were positive. Then, since the $\mathscr{Z}$, are all effective (i.e., in the sense of divisors, not in the sense of an "effective" form of the Mordell conjecture), there would exist a constant $K \in \mathbb{N}$ such that the inequality

$$
\left(*_{1}\right) \Theta \leq K \lambda
$$

would hold on $\mathscr{G}$. Now suppose that our curve $f: C \rightarrow B$ is fixed and that we have a fixed line bundle $\mathscr{L}$ on $C$ of relative degree $d-1$ for some large $d$. Then we have a diagram:

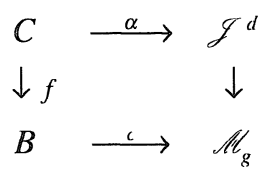

where $\alpha$ is defined on an $S$-section $\tau: S \rightarrow C_{S}$ by associating to $\tau$ the line bundle $\mathscr{L}(\tau)$ (where $\tau$ is regarded as a relative divisor) of relative degree $d$ on $C_{S}$. Now $\alpha \Theta$ is relatively ample on $C$ and hence defines a height $h_{\Theta}(-)$ on rational points. Now if we pull back the Grassmann bundle $\alpha^{\prime}(\mathscr{G} \rightarrow \mathscr{J})$ to a bundle over $C$, this Grassmann bundle, which a priori is locally trivial only in the étale 
topology, will, in fact, be locally trivial for the Zariski topology; thus, if we are given a section $\sigma$ of $f$, and pull-back this Grassmann bundle further via $\sigma$ to a Grassmann bundle over $B$, this bundle over $B$ will always have a section over $B$. Thus we can pull back the relation $\left(*_{1}\right)$ via $\alpha \circ \sigma$ to obtain:

$$
\left(*_{2}\right) h_{\Theta}(\sigma) \leq K \operatorname{deg}_{A}\left(c^{\prime} \lambda\right)
$$

Moreover, this holds (with the same $K$ ) over any number field $F$ and thus gives us a relation of the same type (only stronger) as that conjectured by Vojta [24], Appendix, $§ 5$, p. 176. In particular, it implies the Mordell conjecture.

§8.6. In fact, however, $\left(*_{2}\right)$ is too strong and it is easy to see that it cannot hold. This is not surprising considering that after all, we derived it using the incorrect assumption that the coefficient of $\mathscr{Z}_{2}$ for $\lambda$ is positive. To form a reasonable conjectural inequality like $\left(*_{2}\right)$, one must somehow involve the discriminant of the base field. Thus, Vojta conjectures:

$$
\left(*_{3}\right) h_{\Theta}(\sigma) \leq K_{1} \operatorname{disc}_{F / Q}+K_{2}
$$

where $K_{1}$ and $K_{2}$ are constants independent of $F$, and $\operatorname{disc}_{F / Q}$ is the "logarithmic discriminant" of loc. cit..

On the other hand, although the derivation of $\S 8.5$ is incorrect, it is very tempting, since it "almost" works in the following metamathematical sense: only the coefficient of $\mathscr{Z}_{2}$ is negative, and, moreover, since $d$, hence $b$, is taken to be very large, the absolute value of the coefficient of $\mathscr{Z}_{2}$ is much smaller than those of the other, positive coefficients. One is, thus, tempted to conjecture that if we write $\mathscr{G}$ for the Grassmann bundle $\sigma^{\prime} \alpha^{\prime}\left(\mathscr{G} \rightarrow \mathscr{J}^{d}\right)$ over $B$, and $\mathscr{D}_{1, \sigma}$ for the various pull-backs of the $\mathscr{D}_{1}$ 's to divisors of $\mathscr{G}_{\sigma}$, then we have the following:

Wish 3. For fixed $F$, we can choose a section $\tau: B \rightarrow G_{\sigma}$ such that $\operatorname{deg}_{A \prime}\left(\tau \mathscr{L}_{2, \sigma}\right)$ is bounded independent of $\sigma$.

as well as:

Wish 4. For variable $F$, there exist $K_{1}, K_{2} \in \mathbf{N}$ such that for any $\sigma$ over any $F$, there exists a section $\tau: B \rightarrow \xi_{\sigma}$ such that $\operatorname{deg}_{A}\left(\tau^{\prime} \mathscr{Z}_{2 . \sigma}\right) \leq K_{1} \operatorname{disc}_{F / Q}+K_{2}$ (so $K_{1}$ and $K_{2}$ are independent of $\sigma$ and $F$ ).

Thus, combined with the reasoning of $\S 8.5$, Wish 3 would imply the ordinary Mordell conjecture, while Wish 4 would imply Vojta's generalization $\left(*_{3}\right)$ of that conjecture. It seems that Wish 3 and Wish 4 might not necessarily be easier to 
prove than the more natural statements that they are alleged to imply, but it is hoped that they might provide an interesting new perspective on the problem.

\section{Bibliography}

[1] Arbarello, E. and Cornalba, M., The Picard Groups of the Moduli Space of Curves, Topology, 26 (1987), 153-171.

[2] Arbarello, E., Weierstrass Points and the Moduli of Curves, Compositio Math., 29 (1974), 325342.

[3] Artin, M., Algebrization of Formal Moduli, I, Global Analysis (Papers in Honor of K. Kodaira), University of Tokyo Press, 1969, 21-71.

[4] Clebsch, A., Zür Theorie der Riemann'schen Fläche, Math. Ann., 6 (1872), 216-230.

[5] Grothendieck, A., Géometrie Formelle et Géometrie Algébrique, Séminaire Bourbaki $\mathbf{1 8 2}$.

[6] Diaz, S. and Donagi, R., Hurwitz Surfaces with Nontrivial Divisors, Algebraic Geometry, Sundance 1988, Contemp. Math., Amer. Math. Soc., 116 (1991), 1-8.

[7] Diaz, S., Donagi, R. and Harbater, D., Every Curve is a Hurwitz Space, Duke J. Math., 59 (1989), 737-746.

[8] Deligne, P. and Mumford, D., The Irreducibility of the Space of Curves of Given Genus, Institute des Hautes Etudes Scientifiques Publications Mathématiques, 36 (1969), 75-109.

[9] Grothendieck, A. and Dieudonné, J., Etude Locale des schémas et des morphismes de schémas, Publ. Math. IHES, 20 (1964), 24 (1965), 28 (1966), 32 (1967).

[10] Faltings, G., Endlichkeitssätze für Abelschen Varietäten über Zahlkörpern, Inv. Math., 73 (1983), 349-366.

[11] Faltings, G. and Chai, C. L., Degenerations of Abelian Varieties, Springer-Verlag, 1990, Chapter 1 .

[12] Grothendieck, A., Fondements de la Géometrie Algébrique. Séminaire Bourbaki 1957-62, Secrétariat Math., Paris, 1962.

[13] Fulton, W., Hurwitz Schemes and the Irreducibility of Moduli of Algebraic Curves, Annals of Math., 90 (1969), 542-575.

[14] Griffiths, P. and Harris, J., Algebraic Geometry, Wiley-Interscience, 1978, pp. 193-211.

[15] Girard, J., Cohomologie non abélienne, Springer-Verlag. 1971.

[16] Harer, J., The Second Homology Group of the Mapping Class Group of an Orientable Surface, Inv. Math., 72 (1982), 221-239.

[17] - The Cohomology of the Moduli Space of Curves, Theory of Moduli (Montecatini Terme, 1985), E. Sernesi (Ed.), Lecture Notes in Math., Springer-Verlag, 1337 (1988).

[18] Harris, J. and Diaz, S., The Geometry of the Severi Variety II: Independence of Divisor Classes and Examples, Algebraic Geometry (Sundance, UT, 1986), Lecture Notes in Math., SpringerVerlag, 1311 (1988), 23-50.

[19] Harris, J. and Mumford, D., On the Kodaira Dimension of the Moduli Space of Curves, Inv. Math., 67 (1982), 23-86.

[20] Hurwitz, A.. Über Riemann'schen Fläche mit gegebenen Verzweigungs-punkten, Math. Ann., 39 (1891), 1-61.

[21] Kato, K., Logarithmic Structures of Fontaine-Illusie, Proceedings of the First JAMI Conference, Johns-Hopkins University Press, 1990, 191-224.

[22] Knudsen, F. F., The Projectivity of the Moduli Space of Stable Curves, II: The Stacks $M_{4 "}$, Math. Scand., 52 (1983), 161-199.

[23] - The Projectivity of the Moduli Space of Stable Curves, III: The Line Bundles on $M_{q n}$, and a Proof of the Projectivity of $\bar{M}_{q n}$ in Characteristic 0, Math. Scand., 52 (1983), 200 212

[24] Lang, S., Introduction to Arakelov Theory, Springer-Verlag, 1988, Appendix by Vojta.

[25] Lüroth, J., Über Verzweigungsschnitte und Querschnitte in einer Riemannschen Fläche, Math. Ann., 4 (1871), 181-184.

[26] Moret-Bailly, L., Exposé II, in Séminaire sur Les Pinceaux Arithmétiques: La Conjecture de Mordell, edited by L. Szpiro, Astérisque, 127 (1985).

[27] Fulton, W., On the Irreducibility of the Moduli Space of Curves, Inv. Math., 67 (1982), 87-88.

[28] Milne, J. S., Étale Cohomology, Princeton University Press, 1980. 
[29] Grothendieck, A. et al., Revêtements étales et Groupe Fondamental, Lecture Notes in Math., Springer-Verlag, 224 (1971).

[30] Vojta, P., Diophantine Approximations and Value Distribution Theory, Lecture Notes in Math., Springer-Verlag, 1239 (1987). 
